\title{
Mesoporous Silica-Supported Metal Oxide-Promoted Rh Nanocatalyst for Selective Production of Ethanol from Syngas
}

Award Number:

DE-FC26-06NT43023

Final Technical Progress Report

Reporting Period:

October 1, 2006 to September 30, 2010

\author{
Submitted By: \\ Iowa State University \\ 1138 Pearson Hall \\ Ames, IA 50011-2207 \\ Principal Investigator: \\ Dr. George A. Kraus \\ University Professor, Department of Chemistry \\ 2759 Gilman Hall \\ Ames, IA 50011-3111 \\ 515-294-7794 / 515-294-0105 (fax) \\ gakraus@iastate.edu
}

Date Report Issued:

December 22, 2010 


\section{DISCLAIMER}

This report was prepared as an account of work sponsored by an agency of the United States Government. Neither the United States Government nor any agency thereof, nor any of their employees, makes any warranty, express of implied, or assumes any legal liability or responsibility for the accuracy, completeness, or usefulness of any information, apparatus, product, or process disclosed, or represents that its use would not infringe privately owned rights. Reference herein to any specific commercial product, process, or service by trade name, trademark, manufacturer, or otherwise does not necessarily constitute or imply its endorsement, recommendation, or favoring by the United States Government or any agency thereof. The views and opinions of authors expressed herein do not necessarily state or reflect those of the United States Government or any agency thereof. 


\title{
Mesoporous Silica-Supported Metal Oxide-Promoted Rh Nanocatalyst for Selective Production of Ethanol from Syngas
}

\author{
Dr. George A. Kraus \\ Iowa State University
}

\begin{abstract}
The objective is to develop a process that will convert synthesis gas from coal into ethanol and then transform the ethanol into hydrogen. Principal investigators from Iowa State University include Dr. George Kraus, Dr. Victor Lin, Marek Pruski, and Dr. Robert Brown. Task 1 involves catalyst development and catalyst scale up. Mesoporous manganese silicate mixed oxide materials will be synthesized, characterized and evaluated. The first-and secondgeneration catalysts have been prepared and scaled up for use in Task 2 . The construction of a high-pressure reactor system for producing synthetic liquid fuel from simulated synthesis gas stream has been completed as the first step in Task 2. Using the first- and second generation catalysts, the reactor has demonstrated the production of synthetic liquid fuel from a simulated synthesis gas stream.
\end{abstract}

Keywords: nanocatalysts, synthesis gas, high-pressure reactor 


\section{TABLE OF CONTENTS}

Disclaimer

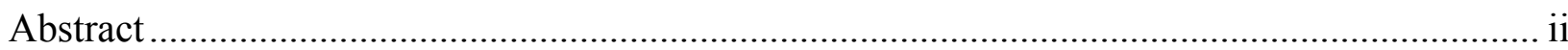

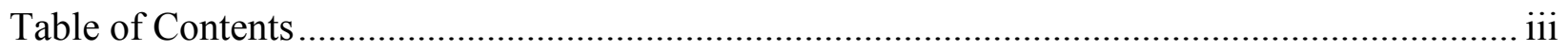

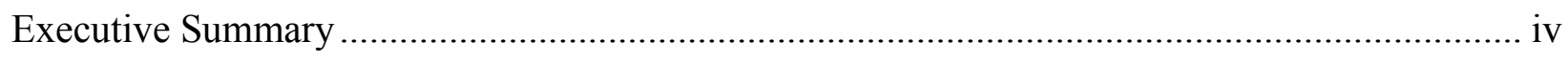

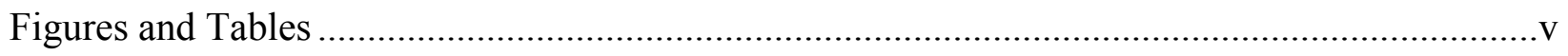

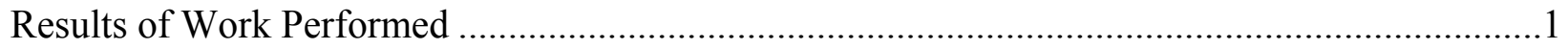

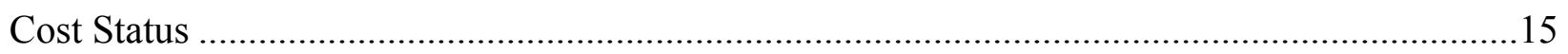

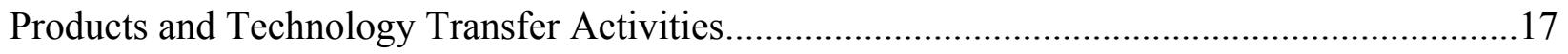

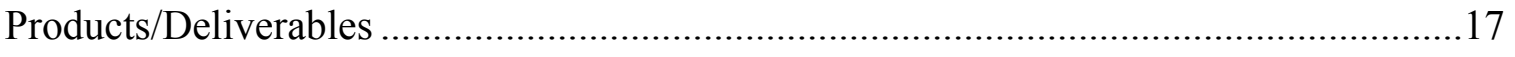

Publications (including Conference Papers) ............................................................. 17

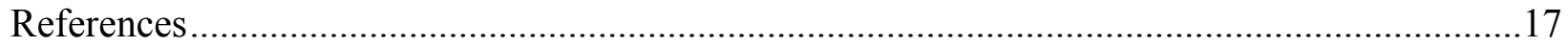

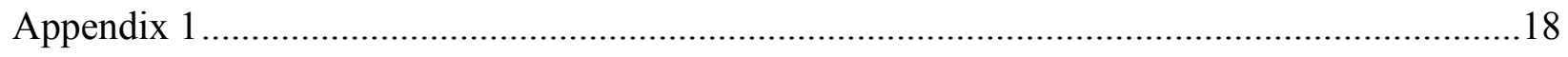

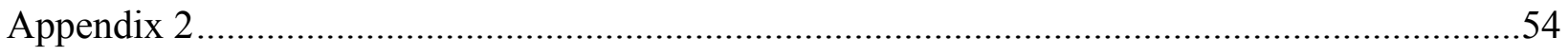

Final Progress Report $\quad$ iii 


\section{EXECUTIVE SUMMARY}

The objective is to develop a process that will selectively convert synthesis gas from coal into ethanol and then transform the ethanol into hydrogen. This effort involves close collaboration between scientists and chemical and mechanical engineers at Iowa State University.

Task 1 involves catalyst development and catalyst scale up. In the past project period, we have refined out synthesis techniques and have scaled up the preparation of catalysts to have sufficient material to be examined by researchers in Task 2 . We have found that the catalytic reaction can be conducted at lower temperatures and lower catalyst loading than reported in the literature. The Lin mesoporous nanoparticle-based catalyst has the best selectivity yet reported.

A high-pressure reactor system for producing synthetic liquid fuel from simulated synthesis gas stream is required as the first step in Task 2. Design and construction of the syngas to ethanol reactor system is complete. The first, second, and third generation Lin catalysts continue to be examined by the reactor system. 


\section{FIGURES AND TABLES}

Figure 1. TEM image and STEM image of MSNRhNPsMn.

Figure 2. XPS of RhNPs and MSNRhNPs before calcination, after calcination, and after reaction.

Figure 3. Flow diagram of the laboratory scale micro-tubular reactor.

Figure 4. Stability testing of $2 \% \mathrm{Rh}-\mathrm{MSN}$ in the micro-tubular reactor.

Figure 5. $\mathrm{CO}$ conversion versus time for the reactions with $\mathrm{Rh}-\mathrm{Mn} / \mathrm{MCN}$ varying GHSV and with varied temperature.

Table 1. Comparison of CO Conversion and Product Profile of Synthesis Gas to Ethanol Reaction Catalyzed by $2 \% \mathrm{Rh}-\mathrm{MSN}$ at $300^{\circ} \mathrm{C}$ and $350{ }^{\circ} \mathrm{C}$ in the Micro-tubular Reactor.

Table 2. Reaction Conditions for $2 \%$ Rh-MSN Tested on Fixed Bed Reactor.

Table 3. Analysis of the Tail Gas and Liquid Products for the Tests Conducted on the Fixed Bed Reactor for the $2 \% \mathrm{Rh}-\mathrm{MSN}$ Catalyst.

Table 4. Conditions For a Set of Reactions Tested on the Fixed Bed Reactor Utilizing 2\%Rh$\mathrm{Mn} / \mathrm{MSN}$ as the Catalyst.

Table 5. Stability of $\%$ Rh-Mn/MSN Under Standard Conditions on the Fixed Bed Reactor.

Table 6. $\mathrm{H}_{2} / \mathrm{CO}$ Ratio Effect on the $2 \% \mathrm{Rh}-\mathrm{Mn} / \mathrm{MSN}$ Catalyst Activity and Selectivity in the Fixed Bed Reactor.

Table 7. Gas Hourly Space Velocity Effect on the 2\%Rh-Mn/MSN Catalyst Activity and Selectivity in the Fixed Bed Reactor.

Table 8. Reaction Temperature Effect on the $2 \% \mathrm{Rh}-\mathrm{Mn} / \mathrm{MSN}$ Catalyst Activity and Selectivity in the Fixed Bed Reactor.

Table 9. Reaction Pressure Effect on the $2 \% \mathrm{Rh}-\mathrm{Mn} / \mathrm{MSN}$ Catalyst Activity and Selectivity in the Fixed Bed Reactor.

Table 10. Different Reaction Conditions for the Optimization of the Activity and Selectivity of $\mathrm{Rh}-\mathrm{Mn} / \mathrm{MCN}$ in the Fixed Bed Reactor.

Table 11. Gas Analysis Results for a Set of Reactions Using Rh-Mn/MCN in the Fixed Bed Reactor.

Table 12. Liquid Analysis Results for a Set of Reactions Using Rh-Mn/MCN in the Fixed Bed Reactor. 


\section{RESULTS OF WORK PERFORMED}

Heterogeneous catalysts have been developed for the conversion of coal-derived synthesis gas (syngas, $\mathrm{CO}$ and $\mathrm{H}_{2}$ ) to ethanol. The objectives of this project were to develop an array of mesoporous catalyst supports for manganese promoted, rhodium catalyzed selective production of ethanol from synthetic gas. During the past few years, we have made tremendous research progress on the three tasks: (1) identify the most favorable catalyst to selectively convert synthetic gas into ethanol, (2) measure reaction rates and yields, determine ethanol selectivity, characterize by products, and (3) provide an analysis of the process with details sufficient to permit a preliminary economic evaluation of the process. In this final report, a detailed explanation of the research challenge to synthesize mesoporous silica and carbon based supports for active rhodium, metal promoted catalysts for the production of ethanol from synthesis gas is given. Progress of the catalyst synthesis is included and a kinetic study of the catalyst systems under different reaction conditions and different reactor set-ups will also be presented and discussed. Specifically, the synthesis of mesoporous silica and mesoporous carbon supports with rhodium nanoparticle catalysts and rhodium nanoparticle with manganese catalysts are described along with the significant material characterizations we completed. In addition to the synthesis and characterization, we described the activity and selectivity of catalysts in our micro-tubular reactor (small scale) and fixed bed reactor (larger scale). After years of hard work, we are proud of the accomplishments of this project, and do believe that this work will provide a solid foundation for the future development of heterogeneous catalysts for the syngas to ethanol process and for the commercialization of this process. Appendix 1 includes thesis chapters from two different graduate students working under the advisement of Prof. Victor S.-Y. Lin on the development, synthesis, characterization, and initial catalytic results of the latest generation $\mathrm{Rh} / \mathrm{Mn}$ supported catalysts.

\section{Catalyst development}

\subsection{Synthesis and characterization of rhodium based, mesoporous silica supported catalysts}

We began the research activities by optimizing the loading of rhodium on mesoporous silica nanoparticle (MSN) materials. The synthesis of MSN can be found in Appendix 1. The rhodium was incorporated into the MSN support by first synthesizing rhodium nanoparticles (RhNPs). These RhNPs were synthesized by a literature preparation where polyvinylpyrrolidone (PVP) was used to stabilize and control the size of the RhNPs. In short, a $7.9 \mathrm{mM}$ solution of PVP was prepared in ethanol and mixed with a solution of $7.1 \mathrm{mM} \mathrm{RhCl}(\mathrm{aq})$. The rhodium salt was reduced and the solvent was removed under reduced atmosphere.

Typically, the as-made RhNPs (480.0 mg, including $40.0 \mathrm{mg} \mathrm{Rh}$ ) were re-dissolved in water $(10.0 \mathrm{~mL}, 0.6 \mathrm{~mol})$ and mixed with a $470 \mathrm{ml}$ aqueous solution of cetyltrimethylammonium bromide (CTAB, $2.0 \mathrm{~g}, 5.5 \mathrm{mmol}), \mathrm{NaOH}(7.0 \mathrm{~mL}$ x $2.0 \mathrm{~mol} / \mathrm{L}, 14.0 \mathrm{mmol})$ at room temperature prior to the adding of $\mathrm{Mn}\left(\mathrm{NO}_{3}\right)_{2} \cdot \mathrm{xH}_{2} \mathrm{O}(104.7 \mathrm{mg}$, including $20.0 \mathrm{mg} \mathrm{Mn})$ and the hydrolysis of tetraethylorthosilicate (TEOS, $10.0 \mathrm{~mL}, 44.8 \mathrm{mmol}$ ) at $80{ }^{\circ} \mathrm{C}$ for $2.0 \mathrm{~h}$. After the hydrolysis of TEOS, the black solid was filtrated and dried under vacuum overnight. This RhNP encapsulated, 
manganese-silicate mesoporous nanoparticle (MSNRhNPsMn) material was characterized after the calcination at $350{ }^{\circ} \mathrm{C}$ in air for $5 \mathrm{~h}$. We ran a complete material characterization of the MSNRhNPsMn including transmission electron microscopy (TEM), scanning transmission electron microscopy (STEM), high and low angle powder X-ray diffraction (XRD), and nitrogen sorption analysis. Prior to testing the activity of the catalyst, MSNRhNPsMn was reduced in a continuous flow of hydrogen $\left(\mathrm{H}_{2}\right)$. Homogeneous distribution of RhNPs in MSNRhNPsMn is observed in the TEM and STEM images (Figure 1a \& b). It is also observed that the RhNP size was maintained at approximately $2 \mathrm{~nm}$. Energy dispersive X-ray (EDX) was used to determine the ratio and loading of $\mathrm{Rh}$ and Mn on MSNRhNPsMn. The loading of Rh and Mn was $1.6 \mathrm{wt} \%$ and $0.8 \mathrm{wt} \%$, respectively with a $\mathrm{Rh}: \mathrm{Mn}$ ratio of $2: 1$.

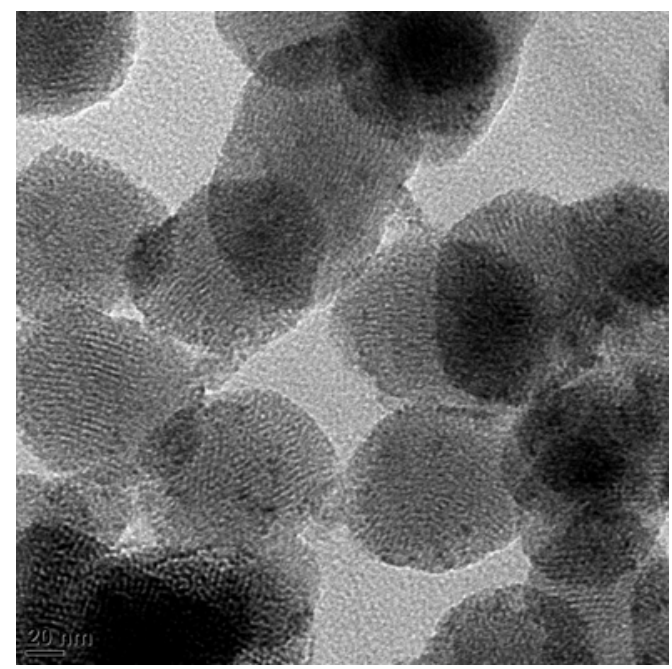

(a)

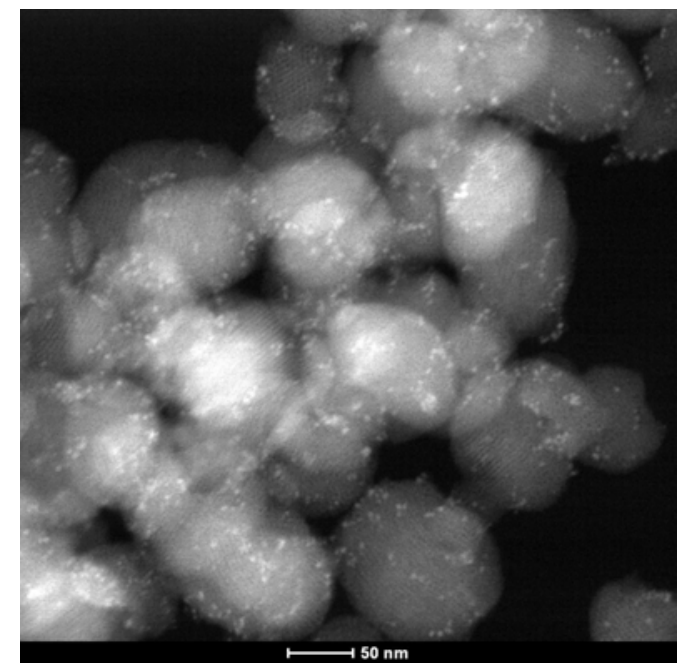

(b)

Figure 1. TEM image (a) and STEM image (b) of MSNRhNPsMn.

In Figure 1a, the tiny black spots (RhNPs) could be observed in addition to the highly ordered parallel channels, which was further confirmed by the STEM image as is seen in Figure 1b. From STEM images of MSNRhNPsMn, it is clear that 1) RhNPs were well-distributed throughout the MSN material and 2) the supported RhNPs was almost the same size, around 2.0 $\mathrm{nm}$ in diameter, as unsupported RhNPs. We discovered that it was challenging to accurately measure the size of small nanoparticles on mesoporous supports because TEM images taken from nanoparticles supported on mesoporous supports often suffer from low contrast due to the decrease of the electron transparency in the supporting material, and this behavior is magnified as particle size decreases. Plus, nanoparticles on mesoporous silica are in different focal planes during TEM imaging. We also synthesized analogous MSN supported rhodium without manganese (MSNRhNPs) to test the dependency of the ethanol selectivity on Mn. These were synthesized exactly the same way, just in the absence of the $\mathrm{Mn}\left(\mathrm{NO}_{3}\right)_{2} \cdot \mathrm{xH}_{2} \mathrm{O}$. The loading of Rh on MSNRhNPs was determined by EDX and we measured a loading of $1.6 \mathrm{wt} \%$ of $\mathrm{Rh}$ in this MSN material based on the atomic ratio between $\mathrm{Si}$ and $\mathrm{Rh}$. 

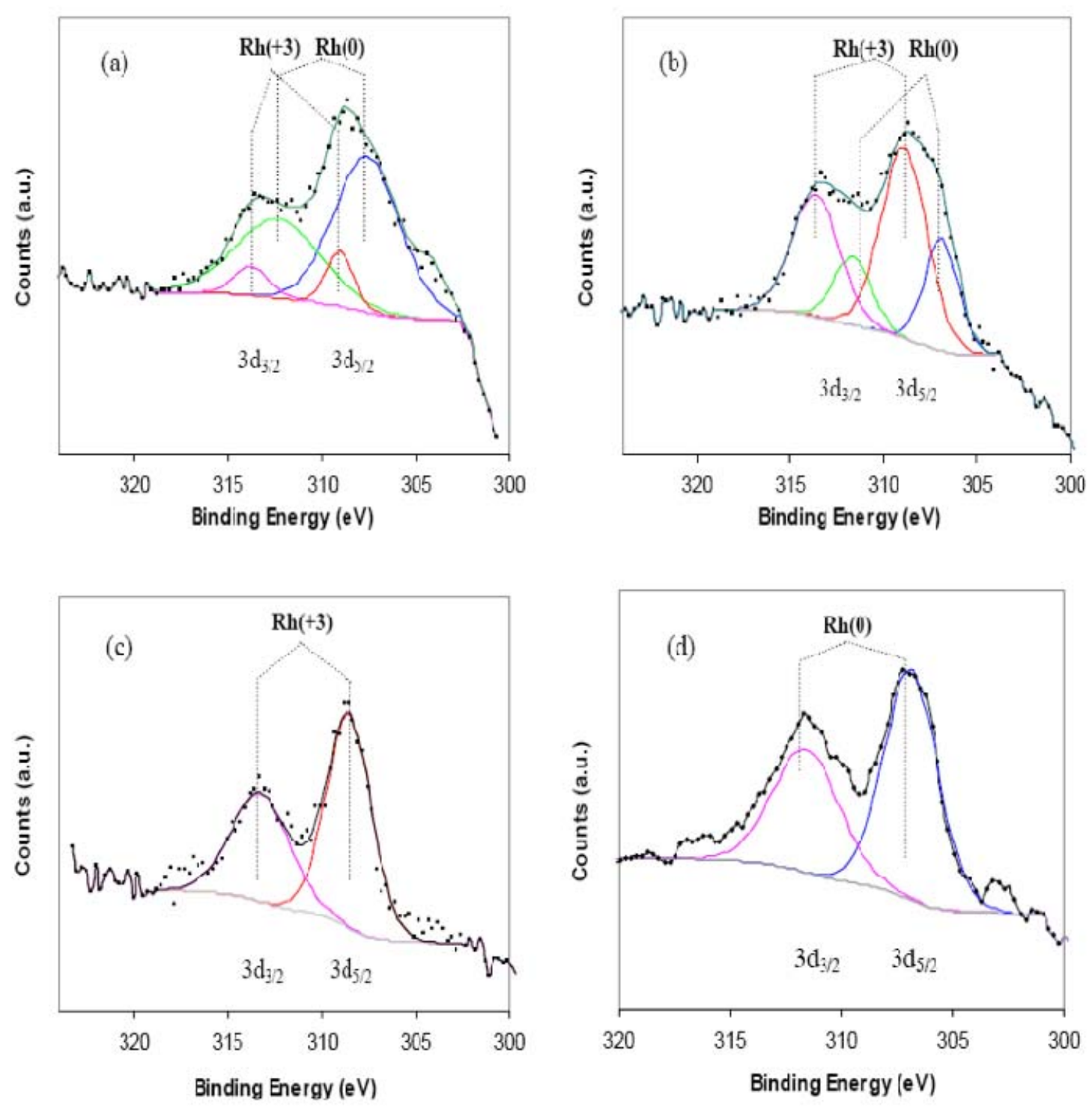

Figure 2. XPS of RhNPs (a) and MSNRhNPs (b) before calcination, (c) after calcination, (d) after reaction.

X-ray photoelectron spectroscopy (XPS) was employed to characterize the oxidation states of $\mathrm{Rh}$ in our RhNPs and MSNRhNPs catalysts on a Perkin-Elmer PHI 5500 XPS spectrometer with a position-sensitive detector, a hemispherical energy analyzer in an ion-pumped chamber (evacuated to $2 \times 10^{-9}$ Torr), and an $\mathrm{Al} \mathrm{K \alpha}(\mathrm{BE}=1486.6 \mathrm{eV}) \mathrm{X}$-ray source at $300 \mathrm{~W}$ with $15 \mathrm{kV}$ acceleration voltage. For all of our experiments, the binding energy of silicon was set at 104.5 $\mathrm{eV}$, which was used as an internal standard for the binding energy of the other elements.

Figure 2 shows the XPS results of RhNPs and MSNRhNPs. Prior to calcination, the Rh $3 \mathrm{~d}_{5 / 2}$ peak $(\sim 308 \mathrm{eV})$ were deconvoluted into two peaks with bonding energies of $307.3 \mathrm{eV}$ and 
$308.9 \mathrm{eV}$, corresponding to the metallic $\mathrm{Rh}(0)$ and the oxidized $\mathrm{Rh}(+3)$, respectively (Figure 2a and 2b). As is shown in Figure 2a, RhNPs are very stable in air at room temperature with $88 \%$ metallic $\mathrm{Rh}(0)$ and $12 \%$ oxidized $\mathrm{Rh}(+3)$. After encapsulating in $\mathrm{MSN}$, there was still $30 \%$ metallic $\mathrm{Rh}(0)$ as is demonstrated in Figure $\mathbf{2 b .}$

After calcining in air at $350{ }^{\circ} \mathrm{C}$ for $5 \mathrm{~h}$, silicon $(\mathrm{Si})$, rhodium $(\mathrm{Rh})$, and oxygen $(\mathrm{O})$ are the three detectable elements from XPS spectroscopy in all of our catalysts reported here. This indicates that the CTAB template and PVP were removed completely from MSNRhNPs. During the calcination step, $\mathrm{Rh}$ was oxidized almost completely to $\mathrm{Rh}_{2} \mathrm{O}_{3}$ (Figure 2c) which can be reduced back to metallic $\mathrm{Rh}(0)$ by $\mathrm{H}_{2}$ during the reaction (Figure 2d). This XPS data indicates that all of the Rh atoms in RhNPs could be accessible to at least $\mathrm{O}_{2}$ and $\mathrm{H}_{2}$ and could potentially be accessible to $\mathrm{CO}$ as well under our reaction conditions.

Manganese was found in all XPS spectroscopic measurements of $\mathrm{Mn}^{\mathrm{n}+}$ modified catalysts (MSNRhNPsMn) (data not shown). However, the oxidation states of Mn species could not be determined because of the low intensity and the relatively small difference between binding energies of $\mathrm{Mn}^{2+}, \mathrm{Mn}^{3+}$ and $\mathrm{Mn}^{4+}$, which decreased the reliability of theoretical fitting. Therefore, it is more accurate to use $\mathrm{Mn}^{\mathrm{n}+}$ to describe the oxidation state of $\mathrm{Mn}$.

\subsection{Synthesis and characterization of rhodium based, mesoporous carbon supported catalysts}

In addition to MSN, we prepared mesoporous carbon nanoparticle (MCN) materials to be used as supports for the rhodium catalysts. These MCN materials were synthesized using a mesoporous aluminosilicate hard-template procedure. The MSN was converted to mesoporous aluminosilicate by adding a solution of $\mathrm{AlCl}_{3}$ to the material. Specifically, the calcined MSN was mixed with distilled water to form silanol groups on the surface of the material, and then completely dried at $150{ }^{\circ} \mathrm{C}$. The dried sample was suspended in an ethanol solution of the anhydrous $\mathrm{AlCl}_{3}(\mathrm{Si} / \mathrm{Al}=20)$. The ethanol solvent was removed under reduced pressure. The dried sample was calcined at $550{ }^{\circ} \mathrm{C}$ to produce the final mesoporous aluminosilicate template.

Ordered mesoporous carbon nanoparticle $(\mathrm{MCN})$ materials were prepared using furfuryl alcohol as the carbon source. Mesoporous aluminosilicate nanoparticles (1 g) were infiltrated with $0.91 \mathrm{~mL}$ of furfuryl alcohol by an impregnation method. The mixture was kept under vacuum at $35^{\circ} \mathrm{C}$ for $1 \mathrm{~h}$. The mixture was heated for $6 \mathrm{~h}$ at $100^{\circ} \mathrm{C}$ for polymerization of furfuryl alcohol and partially carbonized at $350{ }^{\circ} \mathrm{C}$ for $3 \mathrm{~h}$ under vacuum. After cooling to room temperature, the sample was charged with an additional $0.58 \mathrm{~mL}$ of furfuryl alcohol, and repeated freeze-vacuum-thaw and polymerization. Further carbonization was accomplished by heating to $900{ }^{\circ} \mathrm{C}$ under vacuum conditions. The pure $\mathrm{MCN}$ product was collected by removing the aluminosilicate template with $\mathrm{HF}$ treatment (10 wt\% $\mathrm{HF}$ in $\mathrm{EtOH} / \mathrm{H}_{2} \mathrm{O}$ solution).

In order to attach the catalytic rhodium and manganese promoter groups to the MCN support the surface had to be chemically functionalized. The procedure to surface functionalize MCN with carboxylic acid (-COOH) groups involved an oxidation step of mixing MCNs with nitric acid. In short, MCN material (300 mg) were placed in a $100 \mathrm{~mL}$ round-bottom flask with $30 \mathrm{~mL}$ nitric acid $(1 \mathrm{M})$ and the solution was refluxed at $110^{\circ} \mathrm{C}$ for $1.5 \mathrm{~h}$, then followed by hot filtration and washed with copious amounts of water until the $\mathrm{pH}$ value of the aqueous filtrate reached 7. 
The as-synthesized carbon material was dried under vacuum at $100{ }^{\circ} \mathrm{C}$ for $8 \mathrm{~h}$ before further usage.

The carboxylic groups on the surface of MCN had to be deprotonated so the MCN support would suspend well in the aqueous solution for the wet impregnation. The MCN-COOH (150 $\mathrm{mg}$ ) was mixed with sodium carbonate $\left(80 \mathrm{mg} \mathrm{Na} \mathrm{CO}_{3} / 20 \mathrm{~mL} \mathrm{H}_{2} \mathrm{O}\right.$ ) and stirred at room temperature for $6 \mathrm{~h}$.

Finally, the catalytic material was produced by impregnating the pores of the MCN with aqueous solutions of $\mathrm{Rh}_{2} \mathrm{Cl}_{2} \mathrm{CO}_{4}$ and $\mathrm{Mn}\left(\mathrm{NO}_{3}\right)_{2}$. Specifically, COONa-MCNs (148.3 mg) were placed in a $50 \mathrm{~mL}$ double-neck round-bottom flask and mixed with $2.8 \mathrm{mg}$ of $\mathrm{Rh}_{2} \mathrm{Cl}_{2} \mathrm{CO}_{4}(2 \mathrm{wt} \%$ of $\mathrm{Rh}$ loading) in $5 \mathrm{~mL}$ toluene under nitrogen. The solution was stirred at $60{ }^{\circ} \mathrm{C}$ for $3 \mathrm{~h}$. After three hours, the prepared $9.6 \mathrm{mg} \mathrm{Mn}\left(\mathrm{NO}_{3}\right)_{2} / 5 \mathrm{~mL}$ ethanol solution (2 $\mathrm{wt} \% \mathrm{Mn}$ loading) was added in the flask. After an additional $3 \mathrm{~h}$, the metal complexed MCNs were filtered, washed with hexane and dried under vacuum overnight. In order to obtain catalytically active Rh$\mathrm{Mn} / \mathrm{MCNs}$, the metal complexed MCNs were reduced under hydrogen with a gas flow rate of $1.67 \mathrm{~mL} / \mathrm{s}$ at $300{ }^{\circ} \mathrm{C}$ for $3 \mathrm{~h}$ with heating ramp rate of $2{ }^{\circ} \mathrm{C} / \mathrm{min}$. The as-synthesized $\mathrm{Rh}-$ $\mathrm{Mn} / \mathrm{MCN}$ was washed with water to remove $\mathrm{NaCl}$ then dried under vacuum at $100{ }^{\circ} \mathrm{C}$ for $8 \mathrm{~h}$ before the conversion of synthesis gas to alcohols.

\section{Catalyst testing in the laboratory scale micro-tubular reactor and a fixed bed reactor}

\subsection{Reactor systems built for the testing of heterogeneous catalysts}

A micro-tubular reactor (Figure 3) has been designed and assembled for testing heterogeneous catalysts developed for the process of converting coal derived syngas to ethanol and by-products including methanol and methane. As detailed below, this reactor consists of a

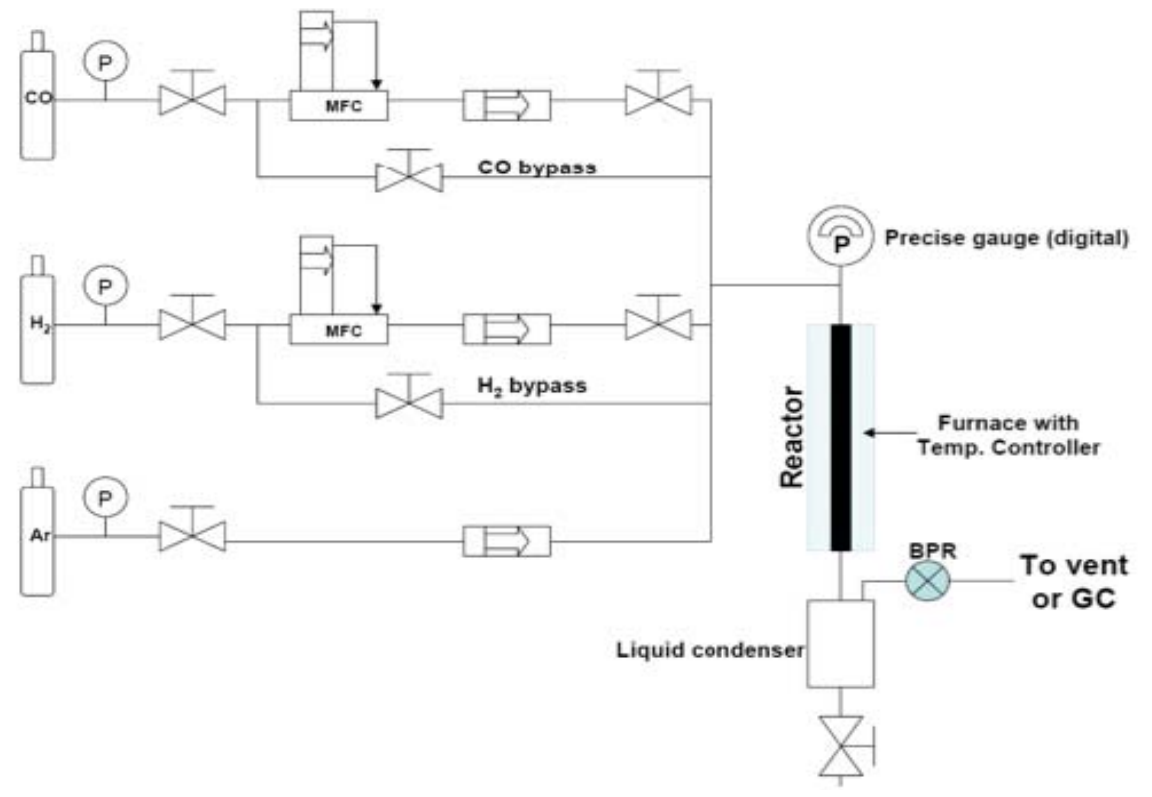

Figure 3. Flow diagram of the laboratory scale micro-tubular reactor.

gas mixing subsystem, a reactor and a condenser. We established a protocol for effective and efficient evaluation of promising catalysts by using this micro-tubular reactor. 
In contrast to the micro-tubular reactor that is used for screening of the catalysts for converting syngas to ethanol, the fixed bed reactor that was built offers additional capability of producing more ethanol by loading more catalysts. However, there are two major challenges associated with the using catalysts in a powder form on this fixed bed reactor.

(1) The local heating inside the catalyst bed resulting from the heat generated from this exothermic reaction.

(2) The large pressure drop along the catalyst bed may create gas shortcuts and consequently lower catalyst utilization.

To resolve these problems, we did some experimentation with the first generation catalyst powder ( $2 \% \mathrm{Rh}-\mathrm{MSN})$. This catalytic material was described in the original proposal. In short, it is a $\mathrm{Rh}$ nanoparticle encapsulated mesoporous silicate material by using an imidazolium IL template. The RhNPs in this case were larger, on the order of $5 \mathrm{~nm}$ in diameter. The MSN support was structurally similar, but synthesized utilizing a different pore template. The first generation catalyst was used for these tests so we could work towards completing multiple tasks concurrently, as we optimized the synthesis of the catalyst, we worked towards optimizing the catalyst conditions. The first catalytic material we synthesized after the first generation was a $2 \% \mathrm{Rh}-\mathrm{Mn} / \mathrm{MSN}$ material. This material was a wet impregnation of $2 \% \mathrm{Rh}-\mathrm{MSN}$ with $\mathrm{Mn}\left(\mathrm{NO}_{3}\right)_{2}$ - $\mathrm{xH}_{2} \mathrm{O}$. We used this supported catalyst material also to test the reactor systems concurrently with the synthesis of the new MSN and MCN supported catalysts described at the beginning of this report. The $2 \% \mathrm{Rh}-\mathrm{MSN}$ catalyst powder was pelletized to make small particles with roughly $1 \mathrm{~mm}$ in size. The following steps are the catalyst preparation procedures for the fixed bed reactors:

1. Approximately $2 \mathrm{~g}$ catalyst powder was pelletized using a Carver press and a die set to make pellets that are $0.3 \mathrm{~cm}$ thick and $\sim 0.75 \mathrm{~cm}$ in diameter. The powder was pressed and held under $5000 \mathrm{lb}$ for 5 minutes.

2. The pellets were broken into smaller pieces.

3. A set of sieves was used to separate catalyst particles of different size and particles with average size between 1.0-1.3 $\mathrm{mm}$ were chosen for this project.

4. Selected catalyst ( $1.08 \mathrm{~g}$ ) was diluted with $5 \mathrm{~g}$ of silica carbide spheres (1.2 $\mathrm{mm}$ in diameter) and loaded into the isothermal zone of the fixed bed reactor.

\subsection{Testing of rhodium based, mesoporous silica supported catalysts (first generation catalyst $-2 \%$ Rh-MSN)}

To examine the catalytic performance of $2 \% \mathrm{Rh}-\mathrm{MSN}, 0.3 \mathrm{~g}$ of catalyst was loaded into the micro-tubular reactor. The catalyst was purged with $\operatorname{Ar}(20 \mathrm{~mL} / \mathrm{min})$ for 30 minutes before reduction by hydrogen $(10 \mathrm{~mL} / \mathrm{min})$ at $300^{\circ} \mathrm{C}$ under 450 -psi pressure for 2 hours. Then the system was purged with synthesis gas (syngas, $\mathrm{CO}$ and $\mathrm{H}_{2}$ ) at a ratio of $2 \mathrm{H}_{2}: 1 \mathrm{CO}$ for 15 minutes before syngas was charged into the system until the pressure reached $450 \mathrm{psi}$. After the reaction reached a steady state at $300{ }^{\circ} \mathrm{C}$ under 450 psi with flow rates of $\mathrm{H}_{2}$ and $\mathrm{CO}$ at 22 and 11 $\mathrm{ml} / \mathrm{min}$, respectively, we analyzed the tail gas via GC periodically. This analysis was the data from which the $\mathrm{CO}$ conversion and the selectivity of gas products, such as methane, $\mathrm{CO}_{2}$, and ethane, were measured and recorded. After the reaction was terminated, liquid samples, which 
were collected in the condenser, were quantitatively analyzed with 1-butanol as an internal standard to determine the selectivity of the liquid product that formed. In addition, the catalytic activity and selectivity of $2 \% \mathrm{Rh}-\mathrm{MSN}$ was tested at $350^{\circ} \mathrm{C}$ and compared the data was compared with catalyst tested at $300{ }^{\circ} \mathrm{C}$. These results are summarized in Table 1.

Table 1 Comparison of CO Conversion and Product Profile of Synthesis Gas to Ethanol Reaction Catalyzed by $2 \% \mathrm{Rh}-\mathrm{MSN}$ at $300{ }^{\circ} \mathrm{C}$ and $350{ }^{\circ} \mathrm{C}$ in the Micro-tubular Reactor

\begin{tabular}{|c|c|c|c|c|c|c|c|}
\hline & \multirow{2}{*}{$\begin{array}{c}\text { Temp } \\
\left({ }^{\circ} \mathrm{C}\right) \\
\end{array}$} & \multirow{2}{*}{$\begin{array}{c}\text { CO conver. } \\
(\%)\end{array}$} & \multicolumn{4}{|c|}{ Product selectivity (mole \%) } & \multirow{2}{*}{$\begin{array}{l}\mathrm{n}_{\mathrm{MeOH}} / \mathrm{n}_{\mathrm{EtOH}} \\
\text { (molar ratio) }\end{array}$} \\
\hline & & & $\mathrm{CH}_{4}$ & $\mathrm{CO}_{2}$ & $\mathrm{MeOH}$ & $\mathrm{EtOH}$ & \\
\hline 1 & 300 & 20.2 & 20.9 & 1.1 & 52.0 & 26.0 & 2.0 \\
\hline 2 & 350 & 28.1 & 55.7 & 7.0 & 24.9 & 12.4 & 2.2 \\
\hline
\end{tabular}

Data listed in Table 1 show that the activity of $2 \% \mathrm{Rh}-\mathrm{MSN}$ at $350{ }^{\circ} \mathrm{C}$ was higher than that at 300 ${ }^{\circ} \mathrm{C}$ (greater CO conversion). However, at $350{ }^{\circ} \mathrm{C}$, the selectivity for liquid alcohol products was significantly decreased from $78 \%$ to $36.3 \%$, this in turn led to an increase in the thermodynamic product, methane.
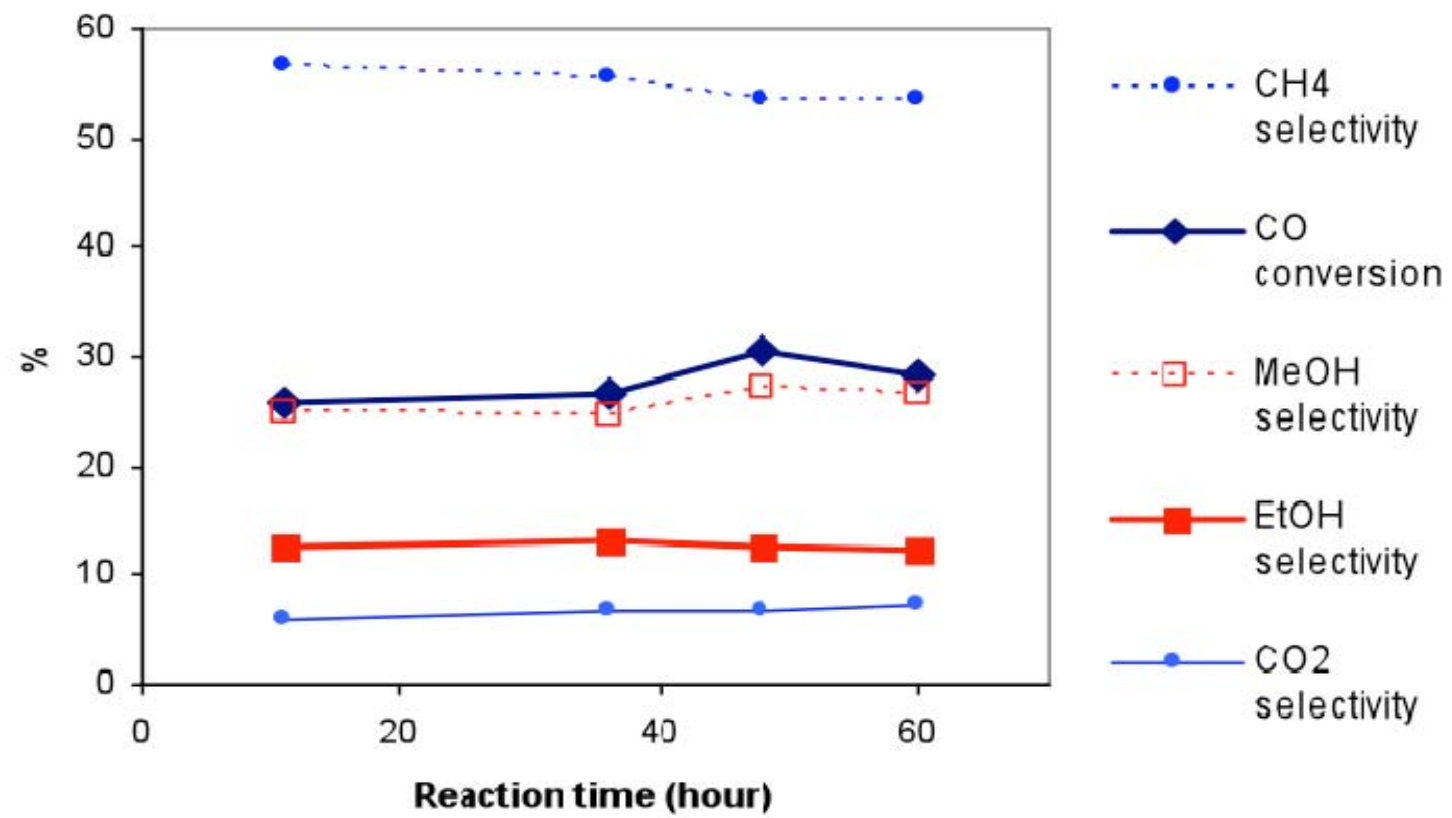

Figure 4. Stability testing of $2 \% \mathrm{Rh}-\mathrm{MSN}$ in the micro-tubular reactor

The stability of the $2 \% \mathrm{Rh}-\mathrm{MSN}$ catalyst was also examined at $350{ }^{\circ} \mathrm{C}$ in the micro-tubular reactor for more than 60 hours and the results are shown in Figure 4. It can be clearly seen that the $\mathrm{CO}$ conversion and the selectivity for major products remained stable, which indicates that the $2 \% \mathrm{Rh}-\mathrm{MSN}$ catalyst is stable in the micro-tubular reactor system, and can be used for additional testing.

Several tests were conducted using the larger scale fixed bed reactor system to evaluate the performance of the $2 \% \mathrm{Rh}-\mathrm{MSN}$ catalyst. All samples of $2 \% \mathrm{Rh}-\mathrm{MSN}$ catalyst used in these 
reactions were reduced at $450 \mathrm{psi}$ and $300{ }^{\circ} \mathrm{C}$ under flowing hydrogen $(100 \mathrm{~mL} / \mathrm{min})$ for 2 hours. Tail gas was sampled and analyzed every one hour throughout the entire reaction period. Liquid products were collected in a condenser after 12 hours at reaction conditions and analyzed by a Varian GC with a flame ionization detector (FID). Reaction conditions and results from these experiments are described in Tables 2 and 3, respectively. The tail gas analysis data was collected after the reaction reached a steady state ( 8 hours for this catalyst) and the liquid analysis data was collected from the products found in the condenser. Both these sets of data are shown in Table 3.

Table 2 Reaction Conditions* for 2\%Rh-MSN Tested on Fixed Bed Reactor

* System pressure (450 psi) and 12-hour reaction time were applied for all reactions

\begin{tabular}{llllll}
\hline $\mathrm{Rxn}$ & $\mathrm{Temp} .\left({ }^{\circ} \mathrm{C}\right)$ & $\mathrm{GHSV}\left(\mathrm{hr}^{-1)}\right.$ & $\mathrm{H}_{2}(\%)$ & $\mathrm{CO}(\%)$ & $\mathrm{He}(\%)$ \\
\hline 3 & 300 & 15000 & 40 & 20 & 40 \\
4 & 300 & 15000 & 30 & 30 & 40 \\
5 & 300 & 30000 & 40 & 20 & 40 \\
6 & 275 & 15000 & 40 & 20 & 40 \\
7 & 325 & 15000 & 40 & 20 & 40 \\
\hline
\end{tabular}

Table 3 Analysis of the Tail Gas and Liquid Products for the Tests Conducted on the Fixed Bed Reactor for the $2 \% \mathrm{Rh}-\mathrm{MSN}$ Catalyst.

\begin{tabular}{|c|c|c|c|c|c|c|c|c|}
\hline \multirow[b]{2}{*}{ Rxn } & \multicolumn{4}{|c|}{ Gas Selectivity } & \multirow[b]{2}{*}{$\begin{array}{l}\mathrm{CO} \text { conv. } \\
(\%)\end{array}$} & \multicolumn{3}{|c|}{ Liquid products } \\
\hline & $\begin{array}{l}\mathrm{CH}_{4} \\
(\%)\end{array}$ & $\begin{array}{l}\mathrm{CO}_{2} \\
(\%)\end{array}$ & $\begin{array}{l}\text { Ethane } \\
(\%)\end{array}$ & $\begin{array}{c}\text { Total } \\
(\%)\end{array}$ & & $\begin{array}{l}\mathrm{MeOH} \\
(\mathrm{wt} \%)\end{array}$ & $\begin{array}{l}\text { EtOH } \\
(w t \%)\end{array}$ & $\begin{array}{l}\mathrm{n}_{\mathrm{MeOH}} \\
/ \mathrm{n}_{\mathrm{EtOH}}\end{array}$ \\
\hline 3 & 15 & 1 & 3 & 19 & 30 & 1.35 & 1.16 & 1.68 \\
\hline 4 & 0 & 2 & 2 & 4 & 11 & 0.88 & 0.56 & 2.25 \\
\hline 5 & 39 & 10 & 9 & 59 & 8 & 5.75 & 3.28 & 2.52 \\
\hline 6 & 0 & 3 & 5 & 8 & 7 & 5.05 & 2.64 & 2.75 \\
\hline 7 & 30 & 11 & 6 & 46 & 23 & 6.65 & 3.59 & 2.65 \\
\hline
\end{tabular}

As one of the original tasks, we were to use a small volume micro-tubular reactor to initially test the catalyst activity and then scale up the synthesis of the catalyst for testing in the higher capacity fixed bed reactor. Test results obtained of the first generation catalyst ( $2 \% \mathrm{Rh}-\mathrm{MSN})$ from the micro-tubular reactor have been successfully repeated in the fixed bed reactor. As shown in Table 1 and Table 3, the results of CO conversion (20.2\% versus $30 \%)$ as well as the total gas selectivity (22\% versus $19 \%)$ were similar, indicating the $2 \% \mathrm{Rh}-\mathrm{MSN}$ catalyst performed well when prepared in the pelletized form for the fixed bed reactor. This consistency in catalyst performance in the two reactor types is a solid basis for the following research in the catalyst scale-up and process development. To increase the selectivity for alcohol products, it has been reported in the literature that a modest $\mathrm{CO}$ conversion is required. As shown in Table 2 and Table 3, several factors affect the efficiency of $\mathrm{CO}$ conversion including the ratio of hydrogen to $\mathrm{CO}$, gas velocity, and reaction temperature. Changing $\mathrm{H}_{2} / \mathrm{CO}$ ratio from 2 to 1 dropped CO conversion from $30 \%$ to $11 \%$, which indicates that the stochiometric ratio of hydrogen and carbon monoxide is imperative for optimizing the catalyst activity. At the same time, higher space velocity and lower reaction temperature also decreased $\mathrm{CO}$ conversion 
significantly, probably because of shorter residence time for reactant molecules and insufficient energy input to overcome activation energy for the reactions, respectively. It was also observed that higher temperature and higher space velocity all contributed to elevated total gas selectivity. Similar to what has been observed in the micro-tubular reactor, there is more carbon transformed to gas products instead of alcohols at higher temperature in the fixed bed reactor. At present time, there is no strong evidence to establish a relationship between reaction conditions and molar ratio of methanol to ethanol in liquid products except for reaction 1, whose ratio is significantly lower than others. More rigorous research is needed in order to obtain accurate mass balance and to correlate kinetics data to reaction mechanisms.

The follow up catalyst to our first generation $2 \% \mathrm{Rh}-\mathrm{MSN}$, was a Mn promoted catalyst that was synthesized the same way with the addition of manganese salt via a wet impregnation method. In order to study the kinetics of the reaction, a set of experimental trials that utilized $2 \% \mathrm{Rh}-\mathrm{Mn} / \mathrm{MSN}$ catalyst were implemented on the fixed bed reactor under various conditions as listed in Table 4 (the parameter varied for a specific reaction has been highlighted in red). For the reaction CSET 21 (experimental trials conducted in the Center for Sustainable Environmental Technologies (CSET) $)$, standard reaction conditions $\left(\mathrm{T}=300{ }^{\circ} \mathrm{C}, \mathrm{P}=450\right.$-psi, Total Flow $=310$ $\mathrm{ml} / \mathrm{min}$ with a $\mathrm{H}_{2} / \mathrm{CO}$ feeding ratio of $2,3.2 \%$ of helium was used in the stream as an internal standard) were used in order to make data comparisons between different reactors and different catalysts. In this report, the effects of reaction variables on the catalytic performance of $2 \% \mathrm{Rh}-$ $\mathrm{Mn} / \mathrm{MSN}$ will be discussed based on the preliminary results obtained.

Table 4. Conditions For a Set of Reactions Tested on the Fixed Bed Reactor Utilizing 2\%Rh$\mathrm{Mn} / \mathrm{MSN}$ as the Catalyst

\begin{tabular}{llllllll}
\hline Rxn & $\begin{array}{l}\text { Temp } \\
\left({ }^{\circ} \mathrm{C}\right)\end{array}$ & $\begin{array}{l}\text { Pres. } \\
(\mathrm{psi})\end{array}$ & $\begin{array}{l}\text { Flow } \\
(\mathrm{ml} / \mathrm{min})\end{array}$ & $\mathrm{H}_{2} / \mathrm{CO}$ & $\mathrm{H}_{2}(\%)$ & $\mathrm{CO}(\%)$ & $\mathrm{He}(\%)$ \\
\hline CSET 21 & 300 & 450 & 310 & 1.86 & 58.86 & 31.69 & 3.43 \\
CSET 22 & 300 & 450 & 620 & 1.89 & 59.94 & 31.79 & 3.42 \\
CSET 23 & 300 & 450 & 465 & 1.9 & 60.29 & 31.66 & 3.39 \\
CSET 24 & 300 & 450 & 310 & 1.0 & 45.47 & 45.40 & 3.33 \\
CSET 25 & 272 & 450 & 310 & $\mathrm{~N} / \mathrm{A}$ & & & \\
CSET 26 & 272 & 450 & 310 & 1.88 & 59.88 & 31.78 & 3.45 \\
CSET 27 & 300 & 450 & 310 & 0.96 & 42.83 & 43.91 & 3.20 \\
CSET 28 & 330 & 450 & 310 & 1.89 & 58.82 & 31.09 & 3.37 \\
CSET 29 & 300 & 450 & 310 & 1.87 & 57.11 & 30.55 & 3.29 \\
CSET 30 & 285 & 450 & 310 & 1.88 & 60.11 & 31.98 & 3.42 \\
CSET 31 & 315 & 450 & 310 & 1.91 & 61.23 & 32.01 & 3.45 \\
CSET 32 & 300 & 450 & 310 & 1.88 & 59.21 & 31.51 & 3.35 \\
CSET 33 & 300 & 350 & 310 & 1.86 & 57.33 & 30.89 & 3.28 \\
CSET 34 & 300 & 550 & 310 & 1.87 & 57.84 & 31.00 & 3.27 \\
\hline
\end{tabular}

* Gas data for CSET 25 were not available, so this reaction was repeated by CSET 26.

The $2 \% \mathrm{Rh}-\mathrm{Mn} / \mathrm{MSN}$ catalyst was reduced at $450 \mathrm{psi}$ and $300{ }^{\circ} \mathrm{C}$ under flowing hydrogen $(100 \mathrm{ml} / \mathrm{min}$ ) for 2 hours before the start of each reaction. Once reduced, $\mathrm{CO}$ and helium were introduced into the system until the desired reaction conditions achieved. Liquid products were condensed in a $250 \mathrm{ml}$ volume liquid/gas separator and collected after 4 hours at reaction 
conditions. A Varian 4300 GC equipped with an FID was used to quantitatively analyze liquid samples. Tail gas was sampled and analyzed every 30 minutes throughout the entire reaction period by a Varian Micro-GC, which was calibrated before each run to ensure reliability.

As can be seen from Table 4, testing of the $\%$ Rh-Mn/MSN catalyst was conducted under different reaction conditions. Among them, CSET 21, CSET 29 and CSET 32 were run with standard conditions to provide comparable data for other trials. CSET 24 and CSET 27 were run with a $\mathrm{H}_{2} / \mathrm{CO}$ ratio of 1 , instead of 2 . CSET 22 and CSET 23 were run with higher gas hourly space velocity (GHSV). The catalyst dependency on temperature was investigated by comparing data obtained from CSET 26, CSET 30, CSET 29, CSET 31 and CSET 28 while pressure effect was explored in reactions CSET 33 and CSET 34.

As reported previously, typically the system reaches a steady state after one-hour at reaction conditions as evidenced by almost unchanged tail gas composition. To make this report concise, we are not showing the time dependence of $\mathrm{CO}$ conversion and selectivity to gases such as methane and carbon dioxide although experimental data are available to support the claim stated above. The results presented in this report are steady state data that were obtained after reacting for 4 hours. Several reaction variables were investigated as detailed in the following.

\section{Repeatability under standard reaction conditions}

Table 5. Stability of $2 \% \mathrm{Rh}-\mathrm{Mn} / \mathrm{MSN}$ Under Standard Conditions on the Fixed Bed Reactor.

\begin{tabular}{llll}
\hline & CSET 21 & CSET 29 & CSET 32 \\
\hline $\mathrm{X}_{\mathrm{CO}}(\%)$ & 29 & 10 & 10 \\
$\mathrm{~S}_{\text {Total }}(\%)$ & 68 & 55 & 55 \\
$\mathrm{~S}_{\text {Methane }}(\%)$ & 55 & 43 & 43 \\
$\mathrm{~S}_{\mathrm{CO} 2}(\%)$ & 2 & 3 & 3 \\
$\mathrm{n}_{\mathrm{MeH}} / \mathrm{n}_{\mathrm{EtOH}}$ & 0.31 & 0.36 & 0.37 \\
\hline
\end{tabular}

Since it has been determined previously that rhodium catalysts demonstrate optimal performance with "standard reaction conditions", these conditions were used for the testing of various catalytic materials. The molar ratio between methanol and ethanol remains relatively stable in the range of 0.3 to 0.4 . Recalling that this ratio was above 2 for other catalysts tested previously, which means the major alcohol product in the liquid was methanol instead of ethanol, there is a significant improvement in term of ethanol selectivity.

At the same time, it is possible that there is structural change during catalyst testing that might degrade catalytic performance, so multiple tests under standard conditions for $2 \% \mathrm{Rh}$ $\mathrm{Mn} / \mathrm{MSN}$ have been implemented. The test results indicate that after the catalyst was subjected to temperatures higher than $330{ }^{\circ} \mathrm{C}$ the performance dropped as evidenced by lower $\mathrm{CO}$ conversion. Although more experimental investigation is needed to relate the catalytic performance to the possible structural or property change during testing, most likely, higher temperature (CSET 28, $\mathrm{T}=330{ }^{\circ} \mathrm{C}$ ) contributed to the performance change. Also observed was that catalytic performance for $2 \% \mathrm{Rh}-\mathrm{Mn} / \mathrm{MSN}$ was repeatable as evidenced by the results obtained from CSET 29 and CSET 32. We speculate that the catalyst was degraded by CSET28 and soon stabilized since no more structural-damaging conditions was involved. So the set of reactions can be split into two parts, before and after CSET28, in which highest temperature in this set of reactions was applied. Any investigation of reaction variables is effective only if the reactions are in the same category where $2 \% \mathrm{Rh}-\mathrm{Mn} / \mathrm{MSN}$ catalyst remains unchanged. 


\section{$\mathrm{H}_{2} /$ CO Ratio Effect}

Table 6. $\mathrm{H}_{2} / \mathrm{CO}$ Ratio Effect on the $2 \% \mathrm{Rh}-\mathrm{Mn} / \mathrm{MSN}$ Catalyst Activity and Selectivity in the Fixed Bed Reactor

\begin{tabular}{llll}
\hline & CSET 21 & CSET 24 & CSET 27 \\
\hline $\mathrm{H}_{2} /$ CO ratio & 2 & 1 & 1 \\
\hline $\mathrm{X}_{\mathrm{CO}}(\%)$ & 29 & 11 & 10 \\
$\mathrm{~S}_{\text {Total }}(\%)$ & 68 & 13 & 12 \\
$\mathrm{~S}_{\text {Methane }}(\%)$ & 55 & 0 & 0 \\
$\mathrm{~S}_{\mathrm{CO} 2}(\%)$ & 2 & 3 & 3 \\
$\mathrm{n}_{\mathrm{MeOH}} / \mathrm{n}_{\mathrm{EtOH}}$ & 0.31 & 0.23 & 0.23 \\
\hline
\end{tabular}

Shown in Table 6 are the data for three reactions conducted with different $\mathrm{H}_{2} / \mathrm{CO}$ ratios. Total conversion of $\mathrm{CO}$ drops significantly from $29 \%$ to $10 \%$ when the $\mathrm{H}_{2} / \mathrm{CO}$ ratio decreases to 1 from 2, which can be explained by hydrogen starvation in the reaction. We also observed that total gas selectivity, a measurement of how much carbon was transformed to gas, dropped significantly indicating more liquid product was produced. More importantly, the methanol/ethanol molar ratio decreases to 0.23 from 0.31 showing better ethanol selectivity. It appears that less hydrogen in the reactant stream might result in more ethanol production. The results obtained from CSET24 have been repeated successfully by CSET27.

\section{GHSV Effect}

Table 7. Gas Hourly Space Velocity Effect on the $2 \%$ Rh-Mn/MSN Catalyst Activity and Selectivity in the Fixed Bed Reactor

\begin{tabular}{llll}
\hline \multicolumn{1}{c}{\begin{tabular}{c} 
GHSV \\
\multicolumn{1}{c}{$\left(\mathrm{L} / \mathrm{g}_{\mathrm{cat}} \mathrm{hr}\right)$}
\end{tabular}} & \multicolumn{1}{c}{$\begin{array}{c}\text { CSET 21 } \\
18.6\end{array}$} & \multicolumn{1}{c}{ CSET 23 } & \multicolumn{1}{c}{ CSET 22 } \\
37.9 & & 37.2 \\
\hline $\mathrm{X}_{\mathrm{CO}}(\%)$ & 29 & 11 & 11 \\
$\mathrm{~S}_{\text {Total }}(\%)$ & 68 & 62 & 58 \\
$\mathrm{~S}_{\text {Methane }}(\%)$ & 55 & 49 & 47 \\
$\mathrm{~S}_{\mathrm{CO} 2}(\%)$ & 2 & 2 & 2 \\
$\mathrm{n}_{\mathrm{MeOH}} / \mathrm{n}_{\mathrm{EtOH}}$ & 0.31 & 0.27 & 0.26 \\
\hline
\end{tabular}

Shown in Table 7 are the data obtained from a set of reactions with different GHSVs, lower to higher from CSET 21 to CSET 23 and CSET 22. Trends that were found are decreases in CO conversion, methane and total gas selectivity with increased GHSV while ethanol selectivity increases a little at the same time. Generally speaking, higher GHSV shortens reactant residence time, and may lower CO consumption and change chemical atmosphere on the catalyst surface, which eventually alters reaction mechanisms. Based on the data, we tentatively conclude that the GHSV (in the investigated range) does not have a significant impact on the catalyst performance.

\section{Reaction Temperature}

Table 8. Reaction Temperature Effect on the $2 \% \mathrm{Rh}-\mathrm{Mn} / \mathrm{MSN}$ Catalyst Activity and Selectivity in the Fixed Bed Reactor 


\begin{tabular}{lccccc}
\hline & CSET 26 & CSET 30 & CSET 29 & CSET 31 & CSET 28 \\
$\begin{array}{l}\text { Temperature } \\
\quad\left({ }^{\circ} \mathrm{C}\right)\end{array}$ & 270 & 285 & 300 & 315 & 330 \\
\hline $\mathrm{X}_{\mathrm{CO}}(\%)$ & 6 & 7 & 10 & 16 & 25 \\
$\mathrm{~S}_{\mathrm{Total}}(\%)$ & 8 & 12 & 55 & 71 & 77 \\
$\mathrm{~S}_{\mathrm{Methane}}(\%)$ & 0 & 0 & 43 & 59 & 63 \\
$\mathrm{~S}_{\mathrm{CO} 2}(\%)$ & 2 & 2 & 3 & 4 & 6 \\
$\mathrm{n}_{\mathrm{MeOH}} / \mathrm{n}_{\mathrm{EtOH}}$ & 0.29 & 0.34 & 0.36 & 0.37 & 0.42 \\
\hline
\end{tabular}

Temperature had the most significant effect on the catalytic performance of $2 \% \mathrm{Rh}-\mathrm{Mn} / \mathrm{MSN}$ of all the reaction variables investigated as is illustrated in Table 8. As reported extensively in the literature, $\mathrm{CO}$ conversion is proportional to the reaction temperature, for example, $\mathrm{CO}$ conversion increased from $6 \%$ at $272^{\circ} \mathrm{C}$ to $25 \%$ at $330^{\circ} \mathrm{C}$. However, it is also observed that the percent of carbon transformed to liquid products decreases significantly since total gas selectivity increases, which means more carbon is transformed to gas products such as light alkanes or carbon dioxide. There is no methane produced at lower temperatures $\left(270^{\circ} \mathrm{C}\right.$ and $\left.285^{\circ} \mathrm{C}\right)$ and more methane is generated at higher temperature as can be seen from the table. Similar behavior for carbon dioxide production is observed, but at a less significant level. It is well known that molecular motion energy increases at higher temperature to overcome the activation energy barrier for the conversion of syngas to ethanol. Since the target product for the project is ethanol, it is desired that all CO is converted to ethanol exclusively, not other compounds. However, there are always byproducts generated according to reaction mechanisms proposed for this process.

Liquid analysis data, as shown in Table 8, indicates that more ethanol is produced at lower temperature in a range of $270-330^{\circ} \mathrm{C}$. This is likely due to decreased occurrences of methanol dissociation on the catalytic surface at lower temperature, which favors carbon chain propagation to form higher alcohols. Similar analysis can be applied to methanol production also: higher temperature increases methane precursor dissociation so surface alkyl species was liberated as methane and carbon dioxide. Based on the above discussion, it appears that a reaction temperature around $300^{\circ} \mathrm{C}$ is optimal for this process.

\section{Reaction Pressure}

Table 9. Reaction Pressure Effect on the 2\%Rh-Mn/MSN Catalyst Activity and Selectivity in the Fixed Bed Reactor

\begin{tabular}{llll}
\hline & CSET 33 & CSET 29 & CSET 34 \\
\hline Pressure (psi) & 350 & 450 & 550 \\
\hline $\mathrm{X}_{\mathrm{CO}}(\%)$ & 9 & 10 & 12 \\
$\mathrm{~S}_{\text {Total }}(\%)$ & 54 & 55 & 57 \\
$\mathrm{~S}_{\text {Methane }}(\%)$ & 42 & 43 & 45 \\
$\mathrm{~S}_{\mathrm{CO} 2}(\%)$ & 3 & 3 & 3 \\
$\mathrm{n}_{\mathrm{MeOH}} / \mathrm{n}_{\mathrm{EtOH}}$ & 0.41 & 0.36 & 0.35 \\
\hline
\end{tabular}

Shown in Table 9 are the data obtained from three reactions conducted under different pressures. This evidence shows that the reaction pressure does not have a significant effect on 
the kinetics of this process although increased $\mathrm{CO}$ conversion and total gas selectivity are observed. Also, the molar ratio of methanol to ethanol seems to decrease with elevated reaction pressure, which can be explained by the shift in reaction equilibrium towards the direction of ethanol production.

The catalytic performance of $2 \% \mathrm{Rh}-\mathrm{Mn} / \mathrm{MSN}$ has been thoroughly investigated at CSET on a fixed bed reactor. Compared to that of other materials such as Rh-Mn/MCN and 2\%Rh-MSN this material showed higher ethanol selectivity as evidenced by low methanol to ethanol molar ratio. At the same time, kinetic studies of this material, indicate that a moderate reaction temperature and lower GHSV favor ethanol production.

\subsection{Testing of Rhodium Based, Mesoporous Carbon Supported Catalysts}

Table 10. Different Reaction Conditions on the Activity and Selectivity of Rh-Mn/MCN in the Fixed Bed Reactor

\begin{tabular}{|c|c|c|c|c|c|c|}
\hline Rxn & $\begin{array}{l}\text { Temp. } \\
\left({ }^{\circ} \mathrm{C}\right)\end{array}$ & $\begin{array}{l}\text { Total Flow } \\
\text { (ml/min) }\end{array}$ & $\begin{array}{l}\text { Hydrogen } \\
(\%)\end{array}$ & $\begin{array}{l}\mathrm{CO} \\
(\%)\end{array}$ & $\mathrm{H}_{2} / \mathrm{CO}$ & $\begin{array}{l}\mathrm{He} \\
(\%)\end{array}$ \\
\hline 8 & $300^{1}$ & 310 & 57.66 & 33.46 & 1.72 & 3.61 \\
\hline 9 & 300 & 155 & N/A & N/A & N/A & $\mathrm{N} / \mathrm{A}$ \\
\hline 10 & 300 & 155 & $\mathrm{~N} / \mathrm{A}$ & N/A & N/A & $\mathrm{N} / \mathrm{A}$ \\
\hline 11 & 300 & 620 & 54.81 & 30.80 & 1.78 & 3.32 \\
\hline 12 & 300 & 465 & 58.58 & 30.53 & 1.91 & 3.31 \\
\hline 13 & $300^{2}$ & 310 & 56.36 & 33.80 & 1.69 & 3.65 \\
\hline 14 & 325 & 310 & 58.64 & 34.30 & 1.71 & 3.64 \\
\hline 15 & 275 & 310 & 58.14 & 34.34 & 1.69 & 3.66 \\
\hline
\end{tabular}

${ }^{1}$ This temperature was controlled between $305^{\circ} \mathrm{C}$ and $310^{\circ} \mathrm{C}$.

${ }^{2}$ This temperature was controlled between $293^{\circ} \mathrm{C}$ and $297^{\circ} \mathrm{C}$.

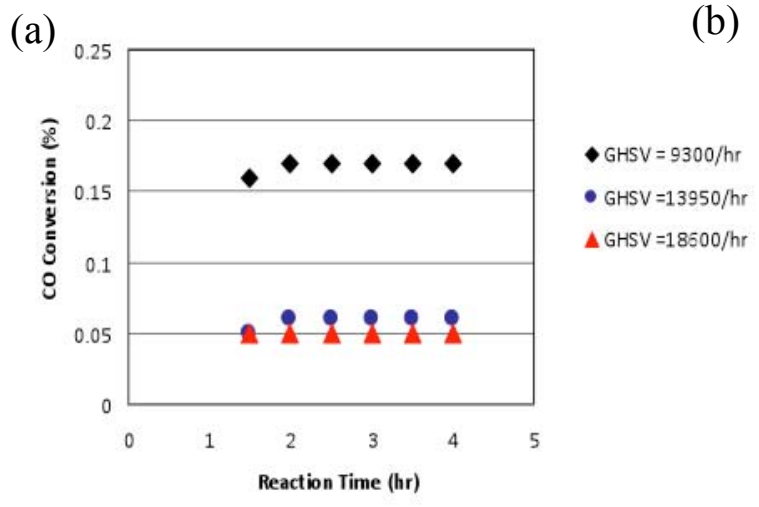

(b)

Figure 5. $\mathrm{CO}$ conversion versus time for the reactions with $\mathrm{Rh}-\mathrm{Mn} / \mathrm{MCN}$ varying $\mathrm{GHSV}$ (Rxn 11, 12 and 13) (a) and with varied temperature (Rxn 15, 14 and 8) (b) 
Corresponding to the catalyst development task, a set of experimental trials that utilized Rh$\mathrm{Mn} / \mathrm{MCN}$ catalyst have been implemented at CSET on the fixed bed reactor with various conditions as listed in Table 10 (the parameter varied for a specific reaction has been highlighted). For reaction 8 , predetermined reaction conditions were used in order to make data comparisons between different reactors and different catalysts. The Rh-Mn/MCN catalyst used in these reactions was reduced at $450 \mathrm{psi}$ and $300{ }^{\circ} \mathrm{C}$ under flowing hydrogen $(100 \mathrm{~mL} / \mathrm{min})$ for 2 hours. Tail gas was sampled and analyzed every 30 minutes throughout the entire reaction period. Liquid products were collected in a condenser after 4 hours at reaction conditions and analyzed by a Varian GC with a FID. Steady state data could not be obtained for reactions CSET 9 and 10 because the hydrogen in the fixed bed reactor from the initial catalyst reduction step was not completely replaced. This data was not recorded because the gas hourly space velocity $(155 \mathrm{ml} / \mathrm{min})$ that those reactions were set too low.

Shown in Figure 5a and $\mathbf{5 b}$ are graphs illustrating the relationship between CO conversion and reaction time for reactions with varied gas hourly space velocity (GHSV) and varied temperature, respectively. After 2-hour, CO conversion did not change indicating the system reached steady state. Shown in Table $\mathbf{1 1}$ are the steady state data for all reactions (except for reaction 9 and 10) from which the following conclusions can be drawn:

1. Higher reaction temperature favors $\mathrm{CO}$ conversion, however, total gas selectivity also increases, which means more $\mathrm{CO}$ has been transformed to methane and $\mathrm{CO}_{2}$.

2. Lower temperature and higher GHSV suppresses $\mathrm{CH}_{4}$ and $\mathrm{CO}_{2}$ formation, however, $\mathrm{CO}$ conversion also decreases significantly.

3. Total gas selectivity decreases at lower temperatures and higher GHSV indicates higher liquid selectivity.

Table 11. Gas Analysis Results for a Set of Reactions Using Rh-Mn/MCN in the Fixed Bed Reactor

\begin{tabular}{|c|c|c|c|c|c|c|c|}
\hline \multirow[b]{2}{*}{ Rxn } & \multicolumn{5}{|c|}{ Selectivity (\%) } & \multirow[b]{2}{*}{$\mathrm{X}_{\mathrm{CO}}(\%)$} & \multirow[b]{2}{*}{$\mathrm{H}_{2} / \mathrm{CO}$} \\
\hline & Methane & $\mathrm{CO}_{2}$ & Ethane & Propane & Total & & \\
\hline 11 & 0.41 & 0.26 & 0.07 & 0.01 & 0.75 & 0.17 & 1.42 \\
\hline 12 & $\mathrm{~N} / \mathrm{A}^{*}$ & & & & & & \\
\hline 13 & $\mathrm{~N} / \mathrm{A}^{*}$ & & & & & & \\
\hline 14 & 0.00 & 0.14 & 0.09 & 0.00 & 0.23 & 0.05 & 1.79 \\
\hline 15 & 0.00 & 0.16 & 0.09 & 0.00 & 0.25 & 0.06 & 1.63 \\
\hline 16 & 0.00 & 0.18 & 0.09 & 0.00 & 0.27 & 0.06 & 1.51 \\
\hline 17 & 0.34 & 0.20 & 0.08 & 0.00 & 0.61 & 0.17 & 1.68 \\
\hline 18 & 0.00 & 0.11 & 0.06 & 0.00 & 0.17 & 0.04 & 2.17 \\
\hline
\end{tabular}

Shown in Table 12 are the analysis results for the liquids obtained from all reactions (except for CSET16, only negligible amount of liquid was collected). Compared to the data that was reported previously for first generation $2 \%$ Rh-MSN catalyst, alcohol concentrations increased substantially, which can be explained by improved catalyst performance since Rh-Mn/MCN was used for this set of reactions. However, total carbon balance for these reactions was in a range of $30-80 \%$, which means significant of amount of reaction products, most likely liquid products 
were not able to be collected. How to improve this will be a focus of the following work for this project.

So far, two catalyst systems have been systematically evaluated on fixed reactor at CSET. The results were consistent with that obtained from micro-tubular reactor. Kinetic studies show that lower $\mathrm{CO}$ conversion, which means mild reaction conditions, is optimal for high alcohol selectivity. This conclusion is consistent with findings. However, the ethanol to methanol ratio, a parameter related to ethanol selectivity, is not comparable to what is reported by other research groups. This could be due to the catalyst itself, and also could be due to catalyst engineering problems such as pelletization or diluting in order to achieve better thermal dissipation.

Table 12. Liquid Analysis Results for a Set of Reactions Using Rh-Mn/MCN in the Fixed Bed Reactor

\begin{tabular}{lllll}
\hline Rxn & $\begin{array}{l}\text { Methanol } \\
\text { (wt\%) }\end{array}$ & $\begin{array}{l}\text { Ethanol } \\
\text { (wt \%) }\end{array}$ & $\begin{array}{l}\text { Propanol } \\
\text { (wt\%) }\end{array}$ & $\begin{array}{l}\text { MeOH/ } \\
\text { EtOH }\end{array}$ \\
\hline 11 & 20.26 & 14.9 & 2.37 & 1.95 \\
12 & 18.29 & 9.45 & 1.04 & 2.78 \\
13 & 7.74 & 3.84 & 0.34 & 2.90 \\
14 & 14.81 & 7.82 & 0.62 & 2.72 \\
15 & 9.62 & 3.81 & 0.39 & 3.63 \\
16 & $\mathrm{~N} / \mathrm{A}$ & $\mathrm{N} / \mathrm{A}$ & $\mathrm{N} / \mathrm{A}$ & $\mathrm{N} / \mathrm{A}$ \\
17 & 16.49 & 8.42 & 1.17 & 2.81 \\
18 & 5.23 & 2.36 & 0.24 & 3.19 \\
\hline
\end{tabular}

\section{Conclusions}

In this project, three major rhodium based catalysts have been successfully developed. Among them, catalysts supported on mesoporous silica show favorable activity and the addition of the Mn promoter improved the performance significantly. However, this set of catalysts produced significant amount of methane, which is not desirable. On the other hand, rhodium catalysts supported on mesoporous carbon produce much less methane probably because of the improvement of thermal distribution along the catalyst bed. Initial attempts to synthesize rhodium catalysts supported on manganese oxides $\left(\mathrm{MnO}_{\mathrm{x}}\right)$ were not successful. We are still attempting to make mesoporous $\mathrm{MnO}_{\mathrm{x}}$ supports that are thermally stable and the structure can withstand extended periods of time at reaction conditions in which high temperature and high pressure are involved. We have had recent success synthesizing mesoporous $\mathrm{MnO}_{\mathrm{x}}$ that has shown improved thermal stability. We are planning on attempting to synthesize RhNP supported $\mathrm{MnO}_{\mathrm{x}}$ catalysts to test on the micro-tubular reactor. We have completed catalytic testing of the first generation catalyst, the manganese doped first generation catalyst, and the mesoporous carbon supported rhodium/manganese (Rh-Mn/MCN) catalysts. Future work consists of completing the catalytic testing of the MSNRhNPs and MSNRhNPsMn. 


\section{COST STATUS}

\begin{tabular}{|c|c|c|c|c|c|c|c|c|}
\hline \multirow{3}{*}{$\begin{array}{c}\text { Baseline Reporting } \\
\text { Quarter }\end{array}$} & \multicolumn{4}{|c|}{ 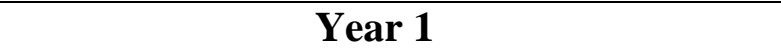 } & \multicolumn{4}{|c|}{ Year 2 (Year 1 no-cost extension) } \\
\hline & \multicolumn{2}{|c|}{ Start: $10 / 1 / 06$} & \multicolumn{2}{|c|}{$9 / 30 / 07$} & \multicolumn{2}{|c|}{ Start: $10 / 1 / 07$} & End: & 30/08 \\
\hline & Q1 & Q2 & Q3 & Q4 & Q5 & $\mathrm{Q6}$ & Q7 & $\mathrm{Q} 8$ \\
\hline $\begin{array}{l}\text { Baseline Cost Plan } \\
\text { (from SF424A) }\end{array}$ & & & & & & & & \\
\hline Federal Share & 495,910 & 185,909 & 185,909 & 185,909 & & & & \\
\hline Non-Federal Share & 46,954 & 46,953 & 46,953 & 46,953 & & & & \\
\hline $\begin{array}{l}\text { Total Planned Costs } \\
\text { (Federal + Non-Federal) }\end{array}$ & 542,864 & 232,862 & 232,862 & 232,862 & & & & \\
\hline Cumulative Baseline & & 775,726 & $1,008,588$ & $1,241,450$ & $1,241,450$ & $1,241,450$ & $1,241,450$ & $1,241,450$ \\
\hline Actual Incurred Costs & & & & & & & & \\
\hline Federal Share & 0 & $75,544.63$ & $\begin{array}{r}197,539.6 \\
5\end{array}$ & $\begin{array}{r}250,029.6 \\
3\end{array}$ & $189,948.90$ & $88,624.23$ & $94,253.60$ & $102,382.96$ \\
\hline Non-Federal Share & 0 & $32,028.22$ & $129,627.4$ & $21,799.78$ & $4,357.56$ & 0 & 0 & 0 \\
\hline Total Incurred Costs & 0 & $107,572.8$ & $\begin{array}{r}327,167.0 \\
0\end{array}$ & $271,829.4$ & $194,306.46$ & $88,624.23$ & $94,253.60$ & $102,382.96$ \\
\hline Cumulative Incurred & & $107,572.8$ & $434,739.9$ & $706,569.2$ & $900,875.81$ & $989,500.0$ & $1,083,753$. & $1,186,136.6$ \\
\hline Variance & & & & & & & & \\
\hline Federal Share & 495,910 & $\begin{array}{r}110,364.3 \\
7\end{array}$ & $11,630.65$ & $64,120.63$ & & & & \\
\hline Non-Federal Share & 46,954 & $23,741.67$ & $82,674.44^{-}$ & $25,153.22$ & & & & \\
\hline $\begin{array}{l}\text { Total Variance - } \\
\text { Quarterly } \\
\text { (Federal + Non-Federal) }\end{array}$ & 542,864 & $\begin{array}{r}134,106.0 \\
4\end{array}$ & - & 38,967.41 & & & & \\
\hline Cumulative Variance & & $\begin{array}{r}676,970.0 \\
4\end{array}$ & $\begin{array}{r}573,848.0 \\
6\end{array}$ & $\begin{array}{r}534,880.6 \\
5\end{array}$ & $340,574.19$ & $\begin{array}{r}251,949.9 \\
6\end{array}$ & $157,696.36$ & $55,313.40$ \\
\hline
\end{tabular}




\section{COST STATUS}

\begin{tabular}{|c|c|c|c|c|c|c|c|c|}
\hline \multirow{3}{*}{$\begin{array}{c}\text { Baseline Reporting } \\
\text { Quarter }\end{array}$} & \multicolumn{4}{|c|}{ Year 3} & \multicolumn{4}{|c|}{ Year 4} \\
\hline & \multicolumn{2}{|c|}{ Start: $\underline{10 / 1 / 08}$} & \multicolumn{2}{|c|}{$9 / 30 / 09$} & \multicolumn{2}{|c|}{ Start: $10 / 1 / 09$} & \multicolumn{2}{|c|}{$9 / 30 / 10$} \\
\hline & Q9 & $\mathrm{Q} 10$ & Q11 & $\mathrm{Q} 12$ & Q13 & & & \\
\hline $\begin{array}{l}\text { Baseline Cost Plan } \\
\text { (from SF424A) }\end{array}$ & & & & & & & & \\
\hline Federal Share & 338,404 & 187,188 & 271,979 & 183,529 & 178,816 & 178,816 & 178,816 & 178,815 \\
\hline Non-Federal Share & 69,245 & 74,796 & 52,940 & 54,312 & 62,877 & 62,877 & 62,877 & 62,877 \\
\hline $\begin{array}{l}\text { Total Planned Costs } \\
\text { (Federal + Non-Federal) }\end{array}$ & 407,649 & 261,984 & 324,919 & 237,841 & 241,693 & 241,693 & 241,693 & 241,692 \\
\hline $\begin{array}{l}\text { Cumulative Baseline } \\
\text { Cost }\end{array}$ & $1,649,099$ & $1,911,083$ & $2,236,002$ & $2,473,843$ & $2,715,536$ & $2,957,229$ & $3,198,922$ & $3,440,614$ \\
\hline Actual Incurred Costs & & & & & & & & \\
\hline Federal Share & $130,850.19$ & $168,056.68$ & $485,293.30$ & $170,436.16$ & $125,499.27$ & $184,514.06$ & $241,395.19$ & $\begin{array}{r}245,631.5 \\
5\end{array}$ \\
\hline Non-Federal Share & $12,058.12$ & $1,124.42$ & $123,691.75$ & $57,016.55$ & $232,740.01$ & 0 & $38,977.85$ & $37,192.30$ \\
\hline $\begin{array}{l}\text { Total Incurred Costs } \\
\text { (Federal + Non-Federal) }\end{array}$ & $142,908.31$ & $169,181.10$ & $608,985.05$ & $227,452.71$ & $358,239.28$ & $184,514.06$ & $280,373.04$ & $\begin{array}{r}282,823.8 \\
5\end{array}$ \\
\hline Cumulative Incurred & $1,329,044$ & $1,498,226$ & $2,107,211$ & $2,334,663$. & $2,692,903$. & $2,877,417$ & $3,157,790$ & $3,440,614$ \\
\hline Variance & & & & & & & & \\
\hline Federal Share & $207,553.81$ & $19,131.32$ & $213,314.30^{-}$ & $13,092.84$ & $53,316.73$ & $-5,698.06$ & $-62,579.19$ & $-66,816.55$ \\
\hline Non-Federal Share & $57,186.88$ & $73,671.58$ & $-70,751.75$ & $-2,704.55$ & $169,863.01^{-}$ & $62,877.00$ & $23,899.15$ & $25,684.7$ \\
\hline $\begin{array}{l}\text { Total Variance - } \\
\text { Quarterly } \\
\text { (Federal + Non-Federal) }\end{array}$ & $264,740.69$ & $92,802.90$ & $284,066.05$ & $10,388.29$ & $116,546.28^{-}$ & $57,178.94$ & $-38,680.04$ & $-41,131.85$ \\
\hline Cumulative Variance & $320,054.09$ & $412,856.99$ & $128,790.94$ & $139,179.23$ & $22,632.95$ & $79,811.89$ & $41,131.85$ & 0 \\
\hline
\end{tabular}




\section{PRODUCTS AND TECHNOLOGY TRANSFER ACTIVITIES}

\section{Products/Deliverables}

Intellectual Property Disclosure to the Office of Intellectual Property and Technology Transfer and the Iowa State University Research Foundation submitted on "A New Mesoporous OxideSupported Metal Catalyst System for Conversion of Synthetic Gas to Alcohols"

Document attached as Appendix 2

\section{Publications (including Conference Papers)}

None

\section{REFERENCES}

See references in Appendix 1. 


\section{APPENDIX 1}

Thesis chapter on the development of Rh-MSN modified by Mn. 


\section{Chapter 4. Mn-Modified and Well-defined Rh nanoparticles Supported on Mesoporous Silica Nanoparticles: Highly-selective Synthesis of Ethanol from Syngas}

Yulin Huang, ${ }^{1}$ Weihua Deng, ${ }^{2}$ Enruo Guo, ${ }^{1}$ Senniang Chen, ${ }^{1}$ Po-Wen Chung, ${ }^{1}$ Brian G. Trewyn, ${ }^{1}$ Victor S.-Y. Lin ${ }^{1, *}$

1. Department of Chemistry and Ames Laboratary-U.S. Department of Energy, Iowa State University, Ames, IA 50011, 2. Center for Sustainable Environmental Technologies, Iowa State University, Ames, IA 5001

ABSTRACT

*Corresponding author.

Well-defined and monodispersed rhodium ( $\mathrm{Rh}$ ) nanoparticles as small as $\sim 2 \mathrm{~nm}$ were synthesized with polyvinylpyrrolidone (PVP) polymer in ethanol. And the as-made PVPstabilized Rh nanoparticles were dispersed and encapsulated in mesoporous silica nanoparticles in situ during the synthesis of this high-surface-area mesoporous silica nanoparticle (MSN) support. Catalytic performance of the MSN-supported Rh nanoparticles (MSNRhNPs) was modified by manganese oxides and studied with CO hydrogenation. MSNRhNPs were active for the $\mathrm{CO}$ hydrogenation and the selectivity to $\mathrm{C} 2$ oxygenates reached the highest point without formation of by-product methanol after the Rh nanoparticles were modified by manganese oxide during the formation of MSN.

Introduction

It is well known that higher alcohols synthesized from natural gas, coal, or biomass can be used as a renewable energy alternative, such as additives to gasoline and an easily transportable source of hydrogen. ${ }^{1-4}$ Because ethanol could not only decrease the dependence on imported cruel oil but also would have a positive environmental impact, synthesis of ethanol selectively from syngas $\left(\mathrm{CO}\right.$ and $\left.\mathrm{H}_{2}\right)$, which can be made from coal or biomass pyrolysis, has been a topic of growing interesting from both industrial and academic points of views. In the catalytic synthesis of ethanol since the $1980 \mathrm{~s},{ }^{5}$ rhodium-based catalysts have been known for decades to be the most selective catalysts for the synthesis of $\mathrm{C}_{2+}$ oxygenates, including ethanol, acetaldehyde and acetic acid, due to the unique $\mathrm{CO}$ adsorption behavior on $\mathrm{Rh}$ surface, although some other transition metal catalysts were reported. ${ }^{2,8-15}$ Most of the attention on Rh- 
based catalysts for ethanol synthesis from syngas was and is still on the influences of catalyst precursors, $^{16-21}$ supports $^{6,22-28}$ and promoters or additives ${ }^{16,21,22,24,29-43}$ on the activity and selectivity. And most of these Rh-based heterogeneous catalysts were made from impregnation of rhodium salt solution which then was followed by calcinations of the as-made solid at high temperature and reduction of rhodium oxides to metallic rhodium particles by hydrogen gas. ${ }^{44-46}$ Usually rhodium particles from this process, including impregnation, calcination and reduction, have a very broad size distribution and vary a lot from batch to batch, because the control of particle size distribution especially on support surface is still very challenging especially at the required high temperature. ${ }^{13}$ And that might be the reason there are only few studies on the size controlling of $\mathrm{Rh}$ particles in $\mathrm{CO}$ hydrogenation. ${ }^{47}$ But, for $\mathrm{CO}$ hydrogenation or any other reactions catalyzed by immobilized $\mathrm{Rh}$ catalysts, Rh particle size controlling should be a very important to adjust the percentage of surface metal atoms that are the only accessible to the reactants. $^{48,49}$ Therefore downsizing $\mathrm{Rh}$ particles, especially to nanometer scale, should be an efficient strategy to increase the reactivity and probably improve the selectivity, due to the dramatically increased percentage of surface metal atoms.

For downsizing metal catalyst particle, colloidal chemistry could be adopted to synthesize monodispersed metal nanoparticle with well-defined particle size in solution. After metal nanoparticle was made in colloidal solution, nanoparticle's dispersion on porous supports by impregnation is required. Mesoporous silica structures have been regarded as ideal catalyst supports due to their high surface area, tunable pore size and highly ordered alignment since its discovery at the beginning of 1990s. ${ }^{27,50-52}$ However, in this methods, in a addition to the difficulty of controlling the homogeneous distribution of metal particles on porous supports surface, rhodium particle growing is still challenging for both chemists and chemical engineer, due to 1) the weak interaction (or physical adsorption) between metal nanoparticle and support surface and 2) the inevitable growth or sintering of catalyst particles.

Here we reported the successful synthesis of $\sim 2.0 \mathrm{~nm}$ Rh particles (RhNPs) in alcoholic solution using a polymer, polyvinylpyrrolidone (PVP), as a nanoparticle stabilizer and subsequent encapsulate the as-made RhNPs in the framework of mesoporous silica nanoparticle (MSN) during in situ of the forming of MSN (Scheme 4-1). Comparing with commonly used MSNsupported Rh catalyst from incipient wetness impregnation of aqueous $\mathrm{RhCl}_{3}$ solution (MSNRh), RhNPs encapsulated in MSN framework (MSNRhNPs) not only have a higher reactivity in CO 
hydrogenation but have also better selectivity to the desired product ethanol. The reactivity of MSNRhNPs in CO hydrogenation and especially the selectivity to ethanol were further improved after it was modified by manganese oxide.

Results and Discussions

Synthesis of PVP-stabilized Rh Nanoparticles and Mesoporous Silica Nanoparticle Framework Encapsulated Rh Nanoparticles. Metal nanoparticles less than $10 \mathrm{~nm}$ that were well-defined and monodispersed with controlled shape have been synthesized by colloid chemistry in recent years. ${ }^{53-55}$ For the synthesis of Rh nanoparticles less than $2.0 \mathrm{~nm}$, there are a few reported methods. ${ }^{48,49,56,57}$ Because PVP is a water soluble polymer, PVP stabilized rhodium nanoparticles can be dispersed homogeneously in water which can be homogeneously distributed in aqueous solution for MSN synthesis. PVP-stabilized RhNPs were synthesized as reported with minor modifications. ${ }^{48,49}$ PVP (Typical $\mathrm{M}_{\mathrm{w}}=29,000$ ) was purchased from Sigma-Aldrich as the nanoparticle stabilizer. A $7.9 \mathrm{mmol} / \mathrm{L}$ PVP solution was prepared by dissolving the polymer into anhydrate ethanol. The PVP ethanolic solution was mixed with $7.1 \mathrm{mmol} / \mathrm{L}$ aqueous $\mathrm{RhCl}_{3}(\mathrm{Rh}$, $38-40 \%$ from Strem Chemicals, Inc.) solution at room temperature, where the mole ratio between PVP and $\mathrm{Rh}^{3+}$ was 10.0. After reduction of rhodium in ethanol, solvent was evaporated by rotavap at $40{ }^{\circ} \mathrm{C}$ and the as-made $\mathrm{Rh}$ nanoparticles (RhNPs) were characterized by transmission electronic miscroscopy (TEM). TEM image (Figure 4-1a) shows that the Rh particles are welldefined and mono-dispersed spheres with $\sim 2.0 \mathrm{~nm}$ in diameter and HRTEM image (Figure 4-1b) combining with diffraction pattern indicates that these tiny RhNPs have the crystalline structures. The as-made RhNPs (480.0 mg, including $40.0 \mathrm{mg} \mathrm{Rh})$ were re-dissolved into water (10.0 mL, $0.6 \mathrm{~mol})$ and mixed with water $(470 \mathrm{~mL}, 26.1 \mathrm{~mol})$, cetyltrimethylammonium bromide (CTAB, $2.0 \mathrm{~g}, 5.5 \mathrm{mmol}), \mathrm{NaOH}(7.0 \mathrm{~mL} \times 2.0 \mathrm{~mol} / \mathrm{L}, 14.0 \mathrm{mmol})$ at room temperature prior to the hydrolysis of tetraethoxyl orthosilicate (TEOS, $10.0 \mathrm{~mL}, 44.8 \mathrm{mmol}$ ) at $80{ }^{\circ} \mathrm{C}$ for 2.0 hours. After the hydrolysis, the grey solid was filtrated and dried under vacuum overnight. The catalytic material MSNRhNPs was made and ready for catalyst characterization, analysis and catalytic tests after the removal of template CTAB and PVP at $350{ }^{\circ} \mathrm{C}$ in air for 5 hours. Before reaction, MSNRhNPs was reduced in continuous $\mathrm{H}_{2}$ flow $(10 \mathrm{~mL} / \mathrm{min})$ at $310{ }^{\circ} \mathrm{C}$ with 450 psi pressure for at least 2 h. $\mathrm{N}_{2}$ adsorption and desorption isotherms (Figure S4-1) show that MSNRhNPs still had a typical mesoporous structure of MSN with a narrow pore size distribution as diameter was 
around $2.4 \mathrm{~nm}$, surface area at $947 \mathrm{~m}^{2} / \mathrm{g}$ and pore volume at $1.0 \mathrm{~mL}$. X-ray powder diffraction (XRD, Figure S4-2) indicated that MSNRhNPs were still full of highly ordered parallel channels, which could be seen clearly from Transmission Electron Microscopy (TEM) images (Figure 42a). In TEM image, a lot of tiny black spots could be seen in addition to the highly ordered parallel channels and these were RhNPs, which was further confirmed by Scanning Transmission Electron Microscopy (STEM) image in Figure 4-2b. From STEM images of MSNRhNPs, it is clear that 1) RhNPs were well-distributed over the mesoporous silica nanoparticle and 2) the supported RhNPs was almost the same size, around $2.0 \mathrm{~nm}$ in diameter, as unsupported RhNPs (as shown in Figure 4-1), although it is very difficult to accurately measure the size of small nanoparticles on mesoporous supports because TEM images taken from nanoparticles supported on mesoporous supports often suffer from low contrast due to the decrease of the supporting material's electron transparency, and this behavior is magnified as particle size decreases, and nanoparticles on mesoporous silica are also in different focal planes during TEM imaging. Energy Dispersive X-ray (EDX) was used to determine the loading of Rh on MSNRhNPs and $1.6 \mathrm{wt} \%$ of $\mathrm{Rh}$ in this material was obtained basing on the atomic ratio between $\mathrm{Si}$ and $\mathrm{Rh}$ (Figure S4-4).

Synthesis of $\mathrm{Rh}-\mathrm{MSN}$ and $\mathrm{Mn}^{\mathrm{n}+}$ Modified $\mathrm{Rh}$ Catalysts through Impregnation Methods. MSNRhNPs' counterparts, Rh-MSN with $1.6 \mathrm{wt} \%$ of $\mathrm{Rh}$ loading, from traditional incipient wetchemistry, impregnation methods, were synthesized (see supporting information) in order to compare their catalytic properties in $\mathrm{CO}$ hydrogenation reactions. For $\mathrm{Rh}$ catalysts in $\mathrm{CO}$ hydrogenation, many promoters were used to improve selectivity to $\mathrm{C}_{2}$ (including ethanol and acetaldehyde) or $\mathrm{C}_{2+}$ oxygenates, ${ }^{2-4}$ and among these reported promoters, $\mathrm{Mn}_{\mathrm{x}} \mathrm{O}_{\mathrm{y}}$ was a very good candidate. ${ }^{34-36,58,59}$ (Here, the oxidation states of Mn species usually could not be accurate and it will be addressed in the flowing studies in this paper.) Therefore, in order to improve the selectivities of MSNRhNPs and Rh-MSN to $\mathrm{C}_{2}$ oxygnates, $\mathrm{Mn}^{\mathrm{n}+}$ modified MSNRhNPs (MSNRhNPs-Mn) and $\mathrm{Mn}^{\mathrm{n}+}$ modified Rh-MSN (Rh-Mn-MSN) were both synthesized by impregnation methods using $\mathrm{Mn}\left(\mathrm{NO}_{3}\right)_{2}$ (see supporting information).

\section{Modification of RhNPs by $\mathrm{Mn}^{\mathrm{n}+}$ during in situ formation of MSN.}

It's a well-known challenge to control the distribution of metal ions on solid surface. Therefore, many catalysts, even with the same chemical composition, have different catalytic performances if they are from different companies or different research groups. For the bimetallic or 
multimetallic catalysts, the situation was even worse due to the difficulty to adjust the interaction between these different metals. In catalyst MSNRhNPs, RhNPs were distributed homogeneously in MSN particles as we can see from Figure 4-3. However, during the $\mathrm{Mn}^{\mathrm{n}+}$ modification procedure, the distribution of $\mathrm{Mn}^{\mathrm{n}+}$ and the interaction between RhNPs and $\mathrm{Mn}^{\mathrm{n}+}$ are still out of control due to the innate disadvantage of impregnation method. In order to achieve a homogeneous distribution of $\mathrm{Mn}^{\mathrm{n}+}$ around RhNPs and the interaction between RhNPs and $\mathrm{Mn}^{\mathrm{n}+}$, we, for the first time, modify RhNPs by $\mathrm{Mn}^{\mathrm{n}+}$ right during the formation of MSN as shown in Scheme 4-2.

Typically, the as-made RhNPs (480.0 mg, including $40.0 \mathrm{mg} \mathrm{Rh}$ ) were re-dissolved into water $(10.0 \mathrm{~mL}, 0.6 \mathrm{~mol})$ and mixed with water $(470 \mathrm{~mL}, 26.1 \mathrm{~mol})$, CTAB (2.0 g, $5.5 \mathrm{mmol}), \mathrm{NaOH}$ $(7.0 \mathrm{~mL} \times 2.0 \mathrm{~mol} / \mathrm{L}, 14.0 \mathrm{mmol})$ at room temperature prior to the adding of $\mathrm{Mn}\left(\mathrm{NO}_{3}\right)_{2} \cdot \mathrm{xH}_{2} \mathrm{O}$ (104.7mg, including $20.0 \mathrm{mg} \mathrm{Mn}$ ) and the hydrolysis of TEOS $(10.0 \mathrm{~mL}, 44.8 \mathrm{mmol})$ at $80{ }^{\circ} \mathrm{C}$ for 2.0 hours. After the hydrolysis of TEOS, the black solid was filtrated and dried under vacuum overnight. The catalytic material MSNRhNPsMn was made and ready for the catalyst characterization after the calcination at $350{ }^{\circ} \mathrm{C}$ in air for 5 hours. Before reaction, MSNRhNPsMn was also reduced in continuous $\mathrm{H}_{2}$ flow as other catalysts. $\mathrm{N}_{2}$ adsorption and desorption isotherms (Figure S4-12), XRD (Figure S4-13) show that MSNRhNPsMn still has the typical highly ordered MSN parallel channel structure with high surface area $\left(\mathrm{S}_{\mathrm{BET}}=878 \mathrm{~m}^{2} / \mathrm{g}\right)$, very narrow pore size distribution $\left(\mathrm{d}_{\mathrm{BJH}}=2.5 \mathrm{~nm}\right)$. TEM and STEM images (Figure 4-3) of MSNRhNPsMn show that RhNPs are distributed homogeneously through the whole structure of MSN and the particle size is still around $2.0 \mathrm{~nm}$ without change after modification with $\mathrm{Mn}^{\mathrm{n}+}$. EDX was used to determine the loading of Rh and Mn on MSNRhNPsMn and $1.6 \mathrm{wt} \%$ of Rh and $0.8 \mathrm{wt} \%$ of $\mathrm{Mn}$ in this material were found, which makes the ratio between $\mathrm{Rh}$ and $\mathrm{Mn}$ around 2.0.

X-ray Photoelectron Spectroscopy (XPS) Study. XPS was used to characterize the oxidation states of $\mathrm{Rh}$ and $\mathrm{Mn}$ in our catalysts on a Perkin-Elmer PHI 5500 XPS spectrometer with a position-sensitive detector, a hemispherical energy analyzer in an ion-pumped chamber (evacuated to $2 \times 10^{-9}$ Torr), and a $\mathrm{Al} \mathrm{K \alpha}(\mathrm{BE}=1486.6 \mathrm{eV}) \mathrm{X}$-ray source at $300 \mathrm{~W}$ with $15 \mathrm{kV}$ acceleration voltage. For all of our experiments, the binding energy of silicon was forced to be $104.5 \mathrm{eV}$ which was used as an internal standard for other elements' binding energy. Figure 4-4 shows the XPS results of RhNPs and MSNRhNPs. Before calcination (Figure4- 4a and 4-4b), the 
$\mathrm{Rh} 3 \mathrm{~d}_{5 / 2}$ peak $(\sim 308 \mathrm{eV})$ could be fit by two peaks with bonding energies of $307.3 \mathrm{eV}$ and 308.9 $\mathrm{eV}$, corresponding to the metallic $\mathrm{Rh}(0)$ and the oxidized $\mathrm{Rh}(+3)$ respectively. As shown in Figure 4-4a, RhNPs was very stable in air at room temperature with $88 \%$ of metallic $\mathrm{Rh}(0)$ and $12 \%$ of oxidized $\mathrm{Rh}(+3)$. After being encapsulated in $\mathrm{MSN}$, there is still $30 \%$ of metallic $\mathrm{Rh}(0)$ as shown in Figure 4-4b. After calcinations in air at $350{ }^{\circ} \mathrm{C}$ for $5 \mathrm{~h}$, from XPS spectroscopy, $\mathrm{Si}$, $\mathrm{Mn}, \mathrm{Rh}$ and $\mathrm{O}$ are the only four detectable elements in all of our catalysts reported here, which indicates that template CTAB and PVP were removed completely from MSNRhNPs surface. With calcination, $\mathrm{Rh}$ was oxidized almost completely to $\mathrm{Rh}_{2} \mathrm{O}_{3}$ (Figure 4-4c) which can be reduced back to metallic $\mathrm{Rh}(0)$ by $\mathrm{H}_{2}$ easily during the reaction(Figure 4-4d). So the XPS data might indicate that all of Rh atoms in RhNPs could be accessible to at least $\mathrm{O}_{2}$ and $\mathrm{H}_{2}$ and might be accessible to $\mathrm{CO}$ as well under our reaction conditions. In any XPS spectroscopy of $\mathrm{Mn}^{\mathrm{n}+}$ modified catalysts, $\mathrm{Mn}^{\mathrm{n}+}$ was found. However, the oxidation states of $\mathrm{Mn}$ species could not be solved because of its lower intensity and the relatively small difference between binding energies of $\mathrm{Mn}^{2+}, \mathrm{Mn}^{3+}$ and $\mathrm{Mn}^{4+}$ (Figure S4-15), which decreases the reliability of fitting theoretically. Therefore, it is better to use $\mathrm{Mn}^{\mathrm{n}+}$ instead of other $\mathrm{Mn}$ species with defined oxidation states here.

Carbon Monoxide hydrogenation Catalyzed by Rh Catalysts. Here a laboratory scale flow and tubular reactor (Figure S4-16) was used for the CO hydrogenation with low surface area SiC as catalyst diluting reagent. Temperature was controlled by a Parr controller (4843) and two type-K thermocouples. Gas flows ( $\mathrm{CO}$ and $\mathrm{H}_{2}$ were all from Praxair and UHP) were regulated by two calibrated mass flow system (Parr mass flow system with Brooks mass flow controllers). Before syngas was charged to the reactor, catalyst was reduced with $10 \mathrm{~mL} / \mathrm{min}_{2}$ flow at 450 psi and $632 \mathrm{~K}$ for two hours. Deionized water was charged into the condenser in order to dissolve most of alcohols from the reaction. Tail gas right after the tubular reactor from the reaction was analyzed on an on-line GC (Varian 3900 with CP-Molsieve 5A (10 m x 0.32 mm x $10 \mu \mathrm{m})$ and CP-PoraBOND Q (50 $\mathrm{m} \times 0.53 \mathrm{~mm} \times 10 \mu \mathrm{m}))$, and a thermal conductivity detector (TCD)) with $5 \mathrm{wt} \% \mathrm{Ar}$ as an internal reference gas. Liquid samples were analyzed on another Varian 3900GC but with flame ionization detector (FID) and a CP-PoraBOND Q (50 m x 0.32 $\mathrm{mm} \times 5 \mu \mathrm{m})$ column.

We found that MSN encapsulated RhNPs catalysts (MSNRhNPs) has higher activity (CO conversion) and better selectivity to $\mathrm{C}_{2}$ oxygenates than that of Rh-MSN made from impregnation methods in CO hydrogenation (Entry 1 and 2 in Table 4-1). And these trends were 
kept very well at different reaction temperatures (Figure S4-17). Since catalytic activity comparison was based on assuming 100\% dispersion for every catalyst, the difference of reactivity was most likely due to the difference between $\mathrm{Rh}$ particles sizes. After reaction at 573 $\mathrm{K}$ for $24 \mathrm{~h}$, TEM images showed that the Rh particles on Rh-MSN (Figure 4-5a) from impregnation methods grew much faster and had a much broader particle distribution (Most of them were larger than $5.0 \mathrm{~nm}$ and some of them even were around $20 \mathrm{~nm}$ in diameter) compared to that of MSNRhNPs (Figure 4-5b) where most of RhNPs were still less than $5 \mathrm{~nm}$ in diameter. The larger $\mathrm{Rh}$ particle in $\mathrm{Rh}-\mathrm{MSN}$ made $\mathrm{CO}$ and $\mathrm{H}_{2}$ accessible surface $\mathrm{Rh}$ atoms less than that in MSNRhNPs with smaller particle size. The higher selectivity to $\mathrm{C}_{2}$ oxygenates of MSNRhNPs might be related to both $\mathrm{Rh}$ particle size and the special interaction between MSN matrix and RhNPs, which is still under investigated in our lab.

After being modified with promoter $\mathrm{Mn}^{\mathrm{n}+}$, as shown in Figure S4-18, MSNRhNPs-Mn had the highest selectivity $(32.4 \%)$ to ethanol and lowest selectivity $(3.7 \%)$ to methanol. And both MSNRhNPs-Mn and Rh-Mn-MSN have higher catalytic activities after being modified with $\mathrm{Mn}^{\mathrm{n}+}$ than that of their unmodified counterparts MSNRhNPs and Rh-MSN respectively (Entry 3 and 4 in Table 4-1). That indicates that manganese oxides not only can improve the selectivity of Rh catalysts to $\mathrm{C} 2$ oxygenates but also can accelerate the $\mathrm{CO}$ hydrogenation. In another words, $\mathrm{Mn}^{\mathrm{n}+}$ not only help to tilt the adsorbed $\mathrm{CO}$ from $\mathrm{Rh}$ to $\mathrm{Mn}$ which is helpful to synthesize ethanol and acetaldehyde according to Bao et. al., ${ }^{58}$ but also participate the hydrogenation of $\mathrm{CO}$ to $\mathrm{HCO}$ which was thought to be the rate limiting step in CO hydrogenation to ethanol according to the density functional theory. ${ }^{60}$ Although the mechanism of Mn effects on CO hydrogenation is still under investigation, it is clear that the close interaction between $\mathrm{Rh}$ and $\mathrm{Mn}$ is necessary for a better catalytic performance of Rh catalyst in $\mathrm{CO}$ hydrogenation, which was further proved by our catalytic tests over MSNRhNPsMn.

As listed in Table 4-1 (Entry 4 and 5), the catalytic activity of MSNRhNPsMn was almost the same as that of MSNRhNPs-Mn, which is because the same RhNPs were used as the catalytic sites in both cases. Although the selectivities to gas product methane were close to each other for MSNRhNPsMn and MSNRhNPs-Mn, interestingly, the selectivity of MSNRhNPsMn to ethanol is much higher than that of MSNRhNPs-Mn as shown in Figure 4-6. Methanol, which usually is one of the main liquid by-products in CO hydrogenation, was kept at very low level through our tests when MSNRhNPsMn was used as catalyst. In the mean time, $\mathrm{CO}_{2}$ production is under GC's 
detection limitation even at $593 \mathrm{~K}$ for MSNRhNPsMn as well. These results indicate that the closer or stronger interaction between promoter $\mathrm{Mn}$ and catalytic site $\mathrm{Rh}$ is necessary to synthesize the ideal product C2 oxygenates such as ethanol and suppress the formation of byproduct methanol. As shown in Figure 4-6, the selectivity of ethanol in tested temperature range from $523 \mathrm{~K}$ to $573 \mathrm{~K}$ reached its highest point and the selectivity of methane touched its lowest point although the CO conversion is only around $9.8 \%$ which is lower than $24.2 \%$ at $543 \mathrm{~K}$.

Conclusions

In summary, we have designed a new Rh-based catalyst in which well-defined rhodium nanoparticles were distributed homogeneously through the whole mesoporous silica particle and could be easily modified by promoters during in situ of the formation of mesoporous silica. The resulting catalyst possesses a high surface area and narrow pore size distribution as normal MSN does. The new manganese modified rhodium catalyst could suppress the formation of by-product methanol efficiently therefore has very high selectivity to the ideal $\mathrm{C}_{2}$ oxygenates in $\mathrm{CO}$ hydrogenation. With the demonstrated better thermostability and better catalytic performance of MSN-encapsulated and Mn-modified rhodium nanoparticles, we defined a novel synthesis and modification method for metallic heterogeneous catalysts.

\section{References}

(1) Farrell, A. E.; Plevin, R. J.; Turner, B. T.; Jones, A. D.; O'Hare, M.; Kammen, D. M. Science (Washington, DC, U. S.) 2006, 311, 506-508.

(2) Fang, K.; Li, D.; Lin, M.; Xiang, M.; Wei, W.; Sun, Y. Catal. Today 2009, 147, 133-138.

(3) Subramani, V.; Gangwal, S. K. Energy Fuels 2008, 22, 814-839.

(4) Spivey James, J.; Egbebi, A. Chem. Soc Rev 2007, 36, 1514-1528.

(5) Takeuchi, K.; Matsuzaki, T.; Arakawa, H.; Sugi, Y. Appl. Catal. 1985, 18, 325-334.

(6) Bhasin, M. M.; Bartley, W. J.; Ellgen, P. C.; Wilson, T. P. J. Catal. 1978, 54, 120-128.

(7) Chuang, S. S. C.; Stevens, R. W., Jr.; Khatri, R. Top. Catal. 2005, 32, 225-232.

(8) Takeuchi, K.; Matsuzaki, T.; Arakawa, H.; Hanaoka, T.; Sugi, Y. Appl. Catal. 1989, 48, 149-157.

(9) Sugi, Y.; Takeuchi, K.; Matsuzaki, T.; Arakawa, H. Chem. Lett. 1985, 1315-1318.

(10) Kintaichi, Y.; Kuwahara, Y.; Hamada, H.; Ito, T.; Wakabayashi, K. Chem. Lett. 1985, 1305-1306. 
(11) Zaman, S. F.; Smith, K. J. Catal. Commun. 2009, 10, 468-471.

(12) Subramanian, N. D.; Balaji, G.; Kumar, C. S. S. R.; Spivey, J. J. Catal. Today 2009, 147, 100-106.

(13) Spivey, J. J.; Egbebi, A. A.; Subramanian, N.; Kumar, N.; Gupta, M. Prepr. Symp. - Am. Chem. Soc., Div. Fuel Chem. 2009, 54, 127-128.

(14) Shi, X.-R.; Jiao, H.; Hermann, K.; Wang, J. J. Mol. Catal. A: Chem. 2009, 312, 7-17.

(15) Gupta, M.; Spivey, J. J. Catal. Today 2009, 147, 126-132.

(16) Jiang, D.; Ding, Y.; Pan, Z.; Li, X.; Jiao, G.; Li, J.; Chen, W.; Luo, H. Appl. Catal., A 2007, 331, $70-77$.

(17) Budge, J. R.; Gates, B. C. 1982, 1, 204-207.

(18) Terreros, P.; Fandos, R.; Granados, M. L.; Otero, A.; Rojas, S.; Vivar-Cerrato, M. A. Stud. Surf. Sci. Catal. 2000, 130D, 3891-3896.

(19) Hanaoka, T.; Kim, W.-Y.; Kishida, M.; Nagata, H.; Wakabayashi, K. Chem. Lett. 1997, 645-646.

(20) Ito, S.-i.; Chibana, C.; Nagashima, K.; Kameoka, S.; Tomishige, K.; Kunimori, K. Appl. Catal., A 2002, 236, 113-120.

(21) Burch, R.; Hayes, M. J. J. Catal. 1997, 165, 249-261.

(22) Jiang, D.; Ding, Y.; Pan, Z.; Chen, W.; Luo, H. Catal. Lett. 2008, 121, 241-246.

(23) Pan, X.; Fan, Z.; Chen, W.; Ding, Y.; Luo, H.; Bao, X. Nat. Mater. 2007, 6, 507-511.

(24) Bastein, A. G. T. M.; Van der Boogert, W. J.; Van der Lee, G.; Luo, H.; Schuller, B.; Ponec, V. Appl. Catal. 1987, 29, 243-260.

(25) Van der Lee, G.; Ponec, V. J. Catal. 1986, 99, 511-512.

(26) Panpranot, J.; Goodwin, J. G., Jr.; Sayari, A. J. Catal. 2002, 211, 530-539.

(27) Martinez, A.; Prieto, G. Top. Catal. 2009, 52, 75-90.

(28) Ma, H.; Yuan, Z.; Wang, Y.; Bao, X. Surf. Interface Anal. 2001, 32, 224-227.

(29) Mo, X.; Gao, J.; Goodwin, J. G. Catal. Today 2009, 147, 139-149.

(30) Haider, M. A.; Gogate, M. R.; Davis, R. J. J. Catal. 2009, 261, 9-16.

(31) Subramanian, N.; Spivey, J. J.; Gao, J.; Mo, X.; Goodwin, J. G.; Torres, W., Jr. Proc. Annu. Int. Pittsburgh Coal Conf. 2008, 25th, 182/1-182/7.

(32) Chen, W.; Ding, Y.; Jiang, D.; Pan, Z.; Luo, H. J. Nat. Gas Chem. 2005, 14, 199-206. 
(33) Yin, H. M.; Ding, Y. J.; Luo, H. Y.; Chen, W. M.; Lin, L. W. Stud. Surf. Sci. Catal. 2004, $147,421-426$.

(34) Ojeda, M.; Granados, M. L.; Rojas, S.; Terreros, P.; Garcia-Garcia, F. J.; Fierro, J. L. G. Appl. Catal., A 2004, 261, 47-55.

(35) Yin, H.; Ding, Y.; Luo, H.; Zhu, H.; He, D.; Xiong, J.; Lin, L. Appl. Catal., A 2003, 243, 155-164.

(36) Yin, H.; Ding, Y.; Luo, H.; Yan, L.; Wang, T.; Lin, L. Energy Fuels 2003, 17, 1401-1406.

(37) Luo, H. Y.; Zhang, W.; Zhou, H. W.; Huang, S. Y.; Lin, P. Z.; Ding, Y. J.; Lin, L. W. Appl. Catal., A 2001, 214, 161-166.

(38) Luo, H.; Zhou, H.; Lin, L.; Liang, D.; Li, C.; Fu, D.; Xin, Q. J. Catal. 1994, 145, 232-234.

(39) Gallaher, G. R.; Goodwin, J. G., Jr.; Huang, C. S.; Houalla, M. J. Catal. 1993, 140, 453463.

(40) Gallaher, G. R.; Goodwin, J. G., Jr.; Guczi, L. Appl. Catal. 1991, 73, 1-15.

(41) Gallaher, G.; Goodwin, J. G., Jr.; Huang, C. S.; Houalla, M. J. Catal. 1991, 127, 719-731.

(42) Chuang, S. S. C.; Pien, S. I.; Narayanan, R. Appl. Catal. 1990, 57, 241-251.

(43) Van der Lee, G.; Schuller, B.; Post, H.; Favre, T. L. F.; Ponec, V. J. Catal. 1986, 98, 522529.

(44) Thomas, J. M.; Thomas, J. W.; Editors Principles and Practice of Heterogeneous Catalysis, 1996.

(45) Satterfield, C. N. Heterogeneous Catalysis in Industrial Practice. 2nd Ed, 1991.

(46) Le Page, J. F. Applied Heterogeneous Catalysis: Design, Manufacture, Use of Solid Catalysts, 1987.

(47) Zhou, S.; Zhao, H.; Ma, D.; Miao, S.; Cheng, M.; Bao, X. Z. Phys. Chem. (Muenchen, Ger.) 2005, 219, 949-961.

(48) Huang, Y.; Li, Y.; Hu, J.; Cheng, P.; Chen, H.; Li, R.; Li, X.; Yip, C. W.; Chan, A. S. C. J. Mol. Catal. A: Chem. 2002, 189, 219-224.

(49) Huang, Y.; Chen, J.; Chen, H.; Li, R.; Li, Y.; Min, L. e.; Li, X. J. Mol. Catal. A: Chem. 2001, 170, 143-146.

(50) Beck, J. S.; Vartuli, J. C.; Roth, W. J.; Leonowicz, M. E.; Kresge, C. T.; Schmitt, K. D.; Chu, C. T. W.; Olson, D. H.; Sheppard, E. W.; et al. J. Am. Chem. Soc. 1992, 114, 1083410843. 
(51) Kresge, C. T.; Leonowicz, M. E.; Roth, W. J.; Vartuli, J. C.; Beck, J. S. Nature (London) 1992, 359, 710-712.

(52) Song, H.; Rioux, R. M.; Hoefelmeyer, J. D.; Komor, R.; Niesz, K.; Grass, M.; Yang, P.; Somorjai, G. A. J. Am. Chem. Soc. 2006, 128, 3027-3037.

(53) Ferrando, R.; Jellinek, J.; Johnston, R. L. Chem. Rev. (Washington, DC, U. S.) 2008, 108, 845-910.

(54) Crooks, R. M.; Lemon, B. I., III; Sun, L.; Yeung, L. K.; Zhao, M. Top. Curr. Chem. 2001, 212, 81-135.

(55) Crooks, R. M.; Zhao, M.; Sun, L.; Chechik, V.; Yeung, L. K. Acc. Chem. Res. 2001, 34, 181-190.

(56) Grass, M. E.; Joo, S. H.; Zhang, Y.; Somorjai, G. A. J. Phys. Chem. C 2009, 113, 86168623.

(57) Huang, W.; Kuhn, J. N.; Tsung, C.-K.; Zhang, Y.; Habas, S. E.; Yang, P.; Somorjai, G. A. Nano Lett. 2008, 8, 2027-2034.

(58) Wang, Y.; Luo, H.; Liang, D.; Bao, X. J. Catal. 2000, 196, 46-55.

(59) Hu, J.; Wang, Y.; Cao, C.; Elliott, D. C.; Stevens, D. J.; White, J. F. Catal. Today 2006, 120, 90-95.

(60) Choi, Y. M.; Liu, P. J. Am. Chem. Soc. 2009, 131, 13054-13061. 


$$
\mathrm{RhCl}_{3}+\mathrm{CH}_{3} \mathrm{CH}_{2} \mathrm{OH}+\mathrm{PVP} \quad \stackrel{80^{\circ} \mathrm{C}_{3} \mathrm{lh} \longrightarrow}{\longrightarrow}
$$

PVP: Polyvinylpyrrolidone

\section{RhNPs}

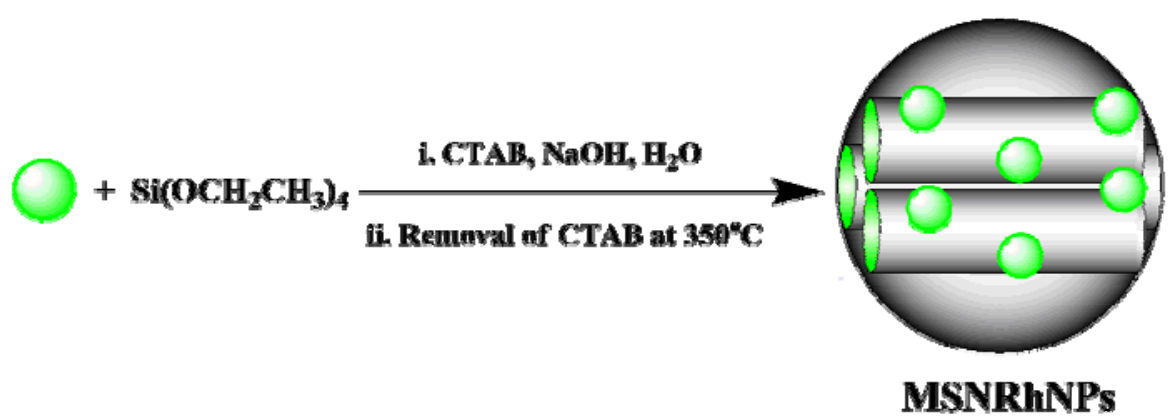

Scheme 4-1. Synthesis of PVP-stabilized Rhodium Nanoparticles and the Subsequent Encapsulation of Rhodium Nanoparticles in the Framework of Mesoporous Silica Nanoparticles.

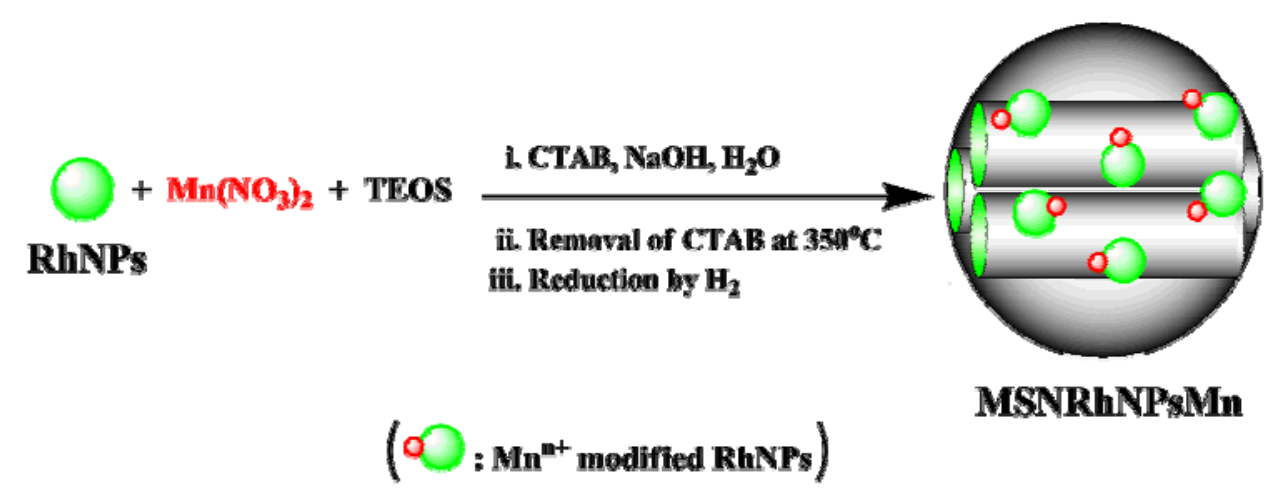

Scheme 4-2. Synthesis of MSNRhNPsMn by co-condensation of $\mathrm{Mn}(\mathrm{NO})_{2}$, RhNPs with TEOS. 


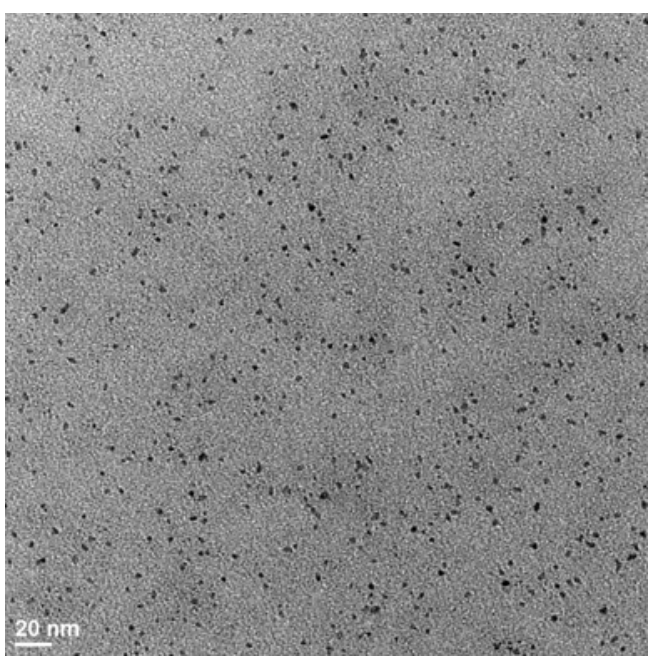

(a)

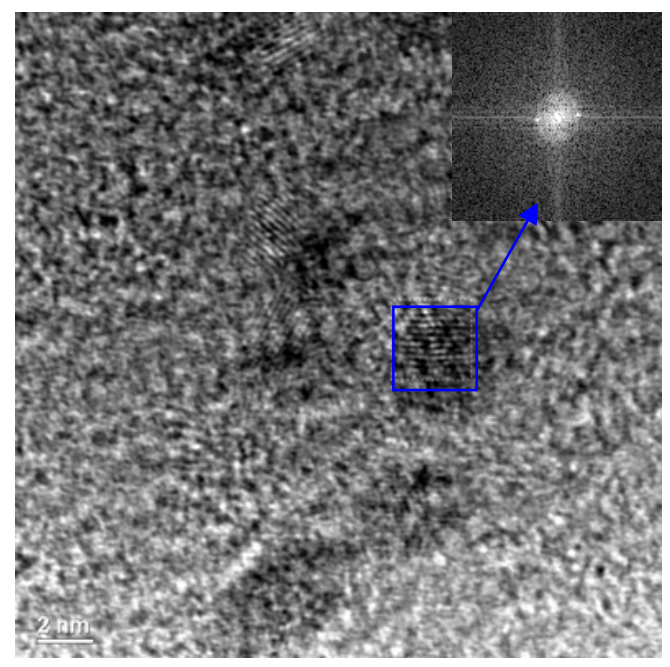

(b)

Figure 4-1. TEM image (a) and HRTEM image (b) of RhNPs.

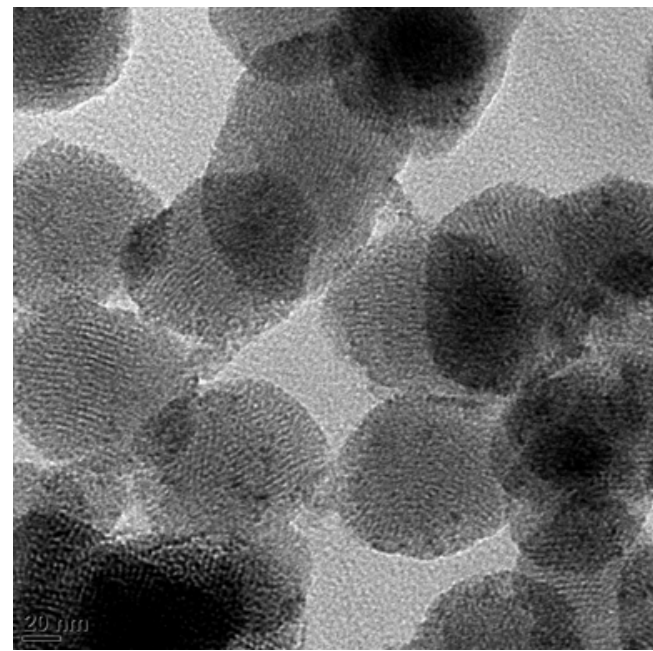

(a)

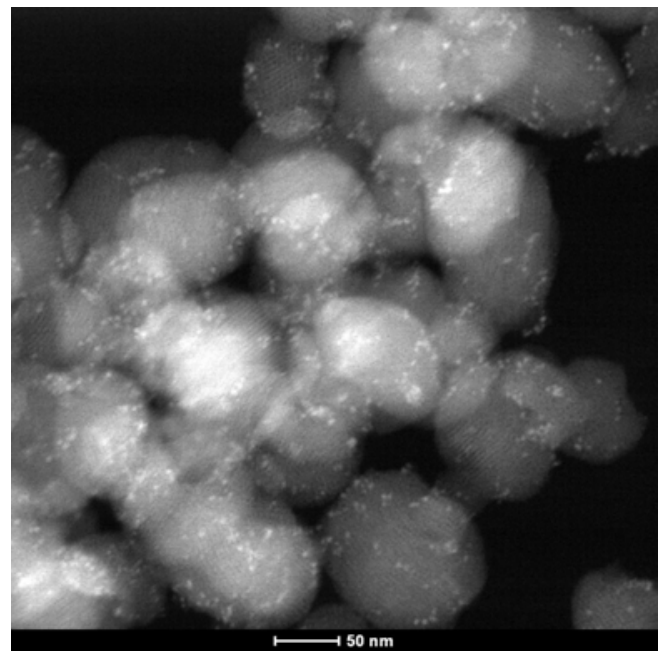

(b)

Figure 4-2. TEM image (a) and STEM image (b) of MSNRhNPs. 

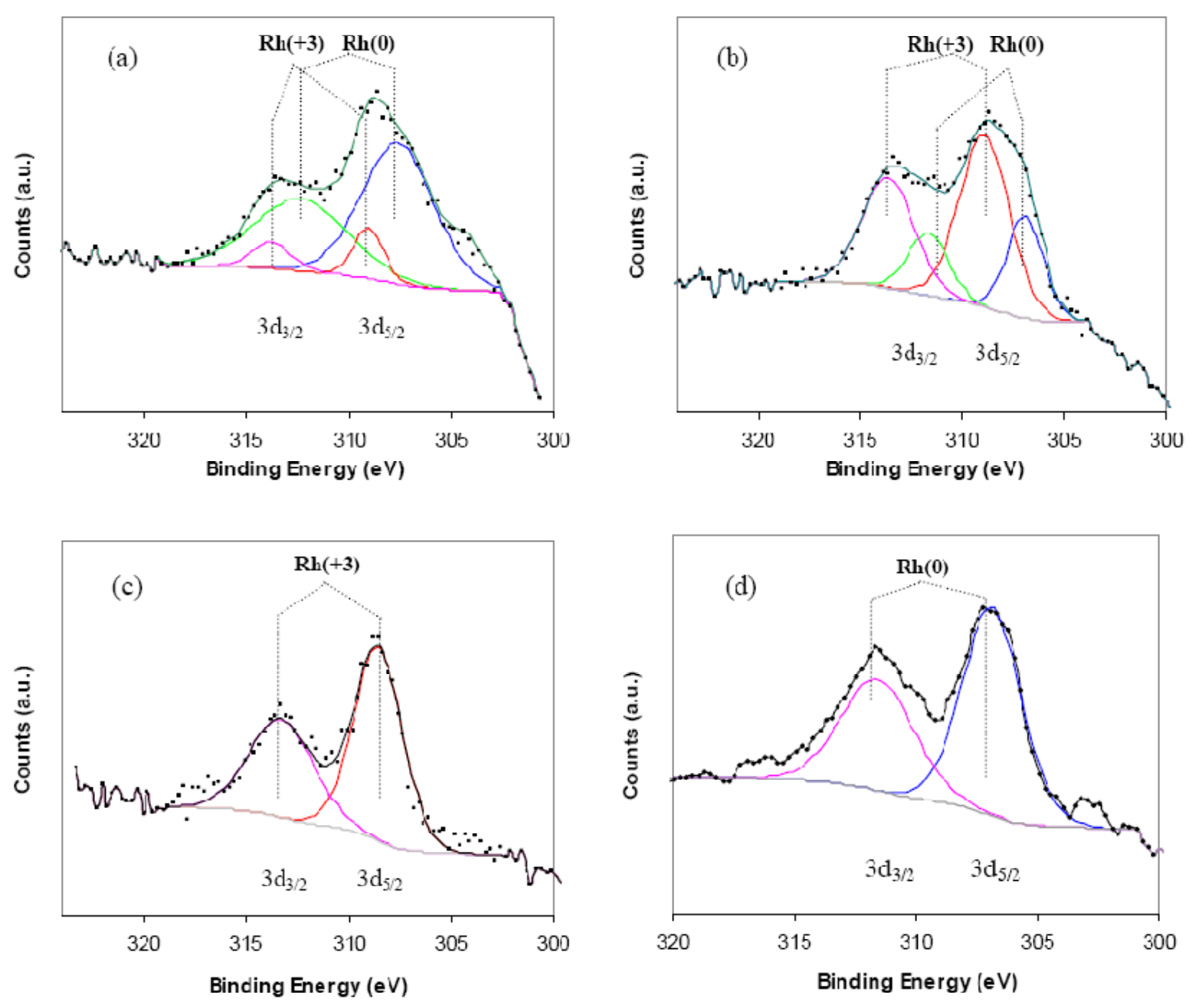

Figure 4-4. XPS of RhNPs (a) and MSNRhNPs (b: before calcination, c: after calcination, d: after reaction. 

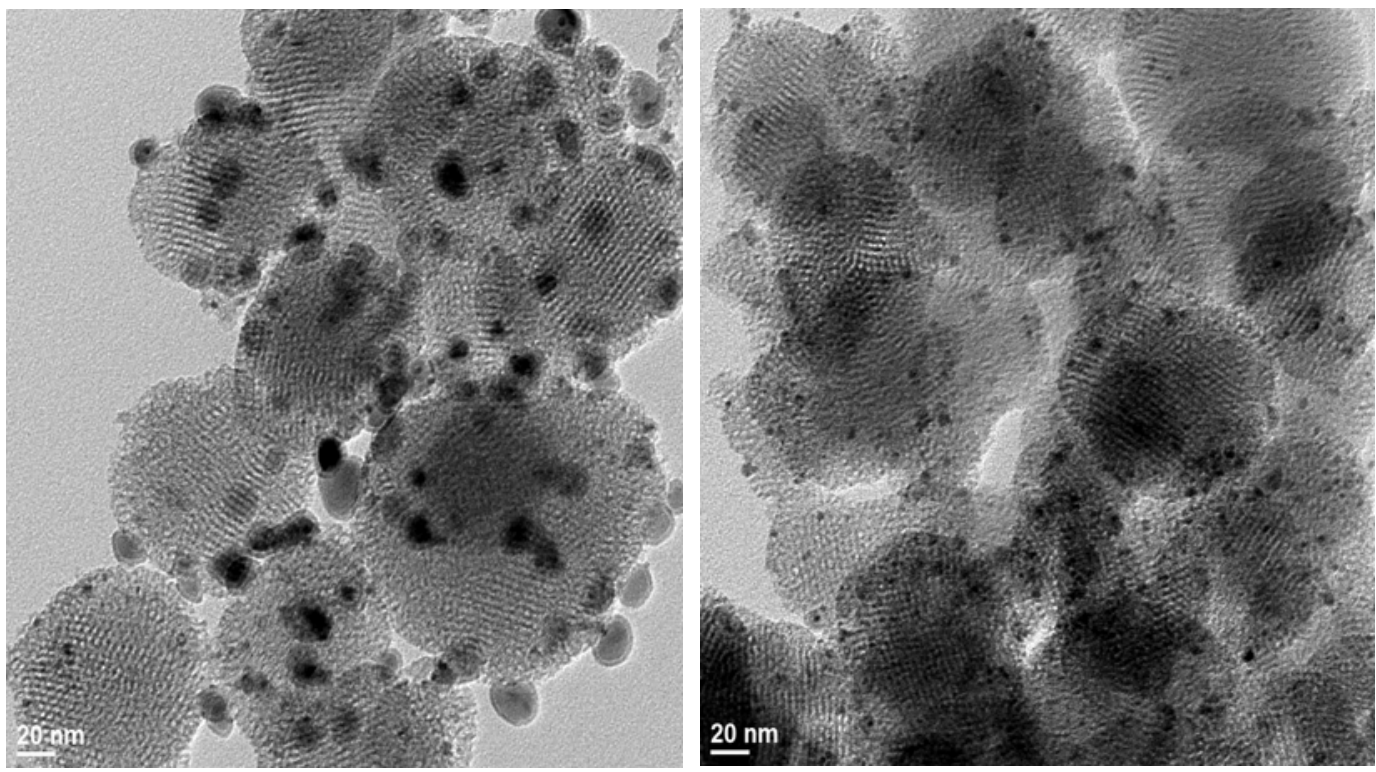

(a)

Figure 4-5. TEM images of Rh-MSN (a) and MSNRhNPs (b) both after reaction at $300{ }^{\circ} \mathrm{C}$ for $24 \mathrm{~h}$.

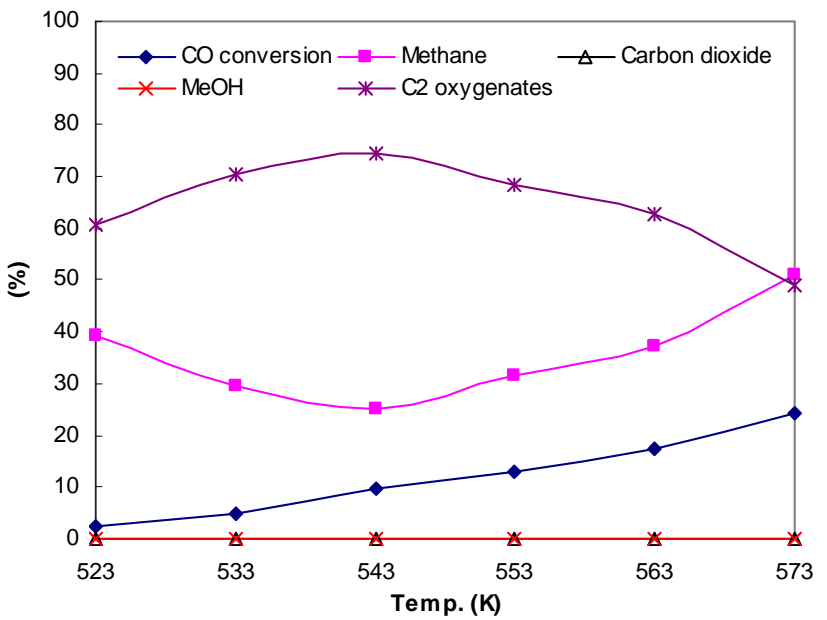

Figure 4-6. Effect of temperature on $\mathrm{CO}$ hydrogenation catalyzed by MSNRhNPsMn.

\begin{tabular}{|l|l|c|l|l|l|l|l|}
\hline & CO conversion & \multicolumn{5}{|c|}{ Selectivity (\%) } \\
\cline { 3 - 8 } & $(\%)$ & $\mathrm{CH}_{4}$ & $\mathrm{CO}_{2}$ & $\mathrm{CH}_{3} \mathrm{OH}$ & $\mathrm{C}_{2}$ & $\mathrm{C}_{2+}$ \\
\hline
\end{tabular}




\begin{tabular}{|c|c|l|l|l|l|l|l|}
\hline 1 & Rh-MSN & 2.7 & 40.8 & 0 & 24.4 & 33.0 & 34.8 \\
\hline 2 & MSNRhNPs & 4.8 & 27.2 & 0 & 17.8 & 53.0 & 55.0 \\
\hline 3 & Rh-Mn-MSN & 13.7 & 63.0 & 1.6 & 3.8 & 24.3 & 31.6 \\
\hline 4 & MSNRhNPs-Mn & 20.2 & 47.0 & 2.5 & 3.7 & 42.2 & 46.8 \\
\hline 5 & MSNRhNPsMn & 24.2 & 51.2 & 0 & 0 & 40.3 & 48.8 \\
\hline
\end{tabular}

* All data were collected at the same conditions $\left(0.3 \mathrm{~g}\right.$ of catalyst with $3.0 \mathrm{~g} \mathrm{SiC}, 300{ }^{\circ} \mathrm{C}, 450 \mathrm{psi}, 11 \mathrm{~mL} / \mathrm{min}$ of $\mathrm{CO}, 22 \mathrm{~mL} / \mathrm{min}$ of $\mathrm{H}_{2}$ ). 
Thesis chapter on the development of Rh-MCN modified by Mn.

\title{
CHAPTER 4. ORDERED MESOPOROUS CARBON NANOPARTICLES AS A SUPPORT FOR THE CONVERSION OF SYNGAS TO ALCOHOL
}

\author{
Po-Wen Chung, Tae-Wan Kim, Yulin Hunag, Weihua Deng, Robert Brown, Victor S.-Y. Lin*
}

\begin{abstract}
The catalytic conversion of synthesis gas (syngas, $\mathrm{CO} / \mathrm{H}_{2}$ ) obtained by gasification of coal or biomass has attracted much attention in the past few years, owing the growing demand for oxygenated products, especially ethanol, which can be used a fuel additive and an energy carrier. Due to the unique carbon monoxide adsorption behavior on Rh, Rh-based catalysts mainly supported by inorganic oxides have been applied to selectively produce the $\mathrm{C}_{2+}$ oxygenates with high activity. However, methane is the most thermodynamically favored product of the $\mathrm{CO}$ hydrogenation and is unlikely to be suppressed unless the size and the dispersion of catalytic metal particles are well regulated over the support. Herein, mesoporous carbon nanoparticle (MCN) possessing well-ordered mesoporosity was developed in Lin group, and ordered mesoporous carbon material was unprecedentedly introduced as a support for the conversion of syngas to alcohol. The catalyst (Rh-Mn/MCN) was characterized by a series of different techniques, including transmission electron microscopy (TEM), powder X-ray diffraction (XRD), $\mathrm{N}_{2}$ sorption analysis, and X-ray photoelectron specstroscopy (XPS) and the catalytic performance was tested in both micro-tubular reactor and pilot-scale fixed bed reactor. Indeed, $\mathrm{Rh}-\mathrm{Mn} / \mathrm{MCN}$ exhibited interesting impact on the suppression of the selectivity of methane and it could be attributed to favorable diffusivity confined by the mesoporosity of MCN.

Introduction

Dwindling petroleum resources, combined with an increased demand for petroleum by emerging economies, as well as environmental concerns about fossil fuels are driving researchers to look for new sources of fuel alternatives. ${ }^{1}$ Ethanol has been used widespreadly as both an energy carrier and a fuel additive. ${ }^{2}$ The catalytic conversion of synthesis gas (syngas, $\mathrm{CO} / \mathrm{H}_{2}$ ) obtained by gasification of coal or biomass has attracted much attention in the past few years, owing the growing demand for oxygenated products, particularly $\mathrm{C}_{2}$ oxygenates such as ethanol, acetaldehyde, and acetic acid. ${ }^{3}$ Rh-based catalysts have been applied to selectively produce the $\mathrm{C}_{2+}$ oxygenates with high activity due to the unique carbon monoxide adsorption behavior on $\mathrm{Rh}^{4-7}$ It is concluded that CO hydrogenation is a structure-sensitive reaction, meaning that the product distribution strongly relies on the metal components, the size of metal particles, the
\end{abstract}


distribution of metal particles over the support and the nature of support. ${ }^{8,9}$ Extensive research efforts have been devoted to investigate the influence of supports and additives including $\mathrm{La}_{2} \mathrm{O}_{3}$, $\mathrm{SiO}_{2}, \mathrm{TiO}_{2}, \mathrm{Al}_{2} \mathrm{O}_{3}, \mathrm{ZrO}_{2}, \mathrm{CeO}_{2}, \mathrm{MgO}, \mathrm{V}_{2} \mathrm{O}_{3}$, alkali metals, $\mathrm{Fe}, \mathrm{Mn}, \mathrm{Ag}$ and Mo on the catalytic activity of $\mathrm{Rh}$ for $\mathrm{CO}$ hydrogenation. In addition, porous silica supported $\mathrm{Rh}$ catalysts were found to affect the catalytic activity and selectivity significantly owing to the control of the size and the distribution of Rh particles. ${ }^{10}$

It is widely concluded that $\mathrm{CO}$ dissociation and hydrogenation to produce $\mathrm{CH}_{\mathrm{x}}$ species is likely the first step for the synthesis of $\mathrm{C}_{2+}$ oxygenates from syngas on Rh-based catalyst. Then the $\mathrm{CH}_{\mathrm{x}}$ species can go through three possible reactions: the formation of $\mathrm{C}_{2}$ oxygenates by $\mathrm{CO}$ insertion, the production of $\mathrm{CH}_{4}$ by hydrogenation, and the chain growth with another $\mathrm{CH}_{\mathrm{x}}$ to produce $\mathrm{C}_{2+}$ hydrocarbons. ${ }^{11}$ Methane is the most thermodynamically favored product and is undesirable economically. In order to suppress methane production and improve the selectivity toward $\mathrm{C}_{2+}$ oxygenates, metal dispersion over the supports has a striking influence for CO hydrogenation. ${ }^{12}$

Carbon is able to exhibit different properties compared to conventional refractory oxide such as $\mathrm{Al}_{2} \mathrm{O}_{3}$ and $\mathrm{SiO}_{2} \cdot{ }^{13}$ Recently Bao's group has demonstrated that carbon nanotubes containing catalytic particles is capable of improving the ethanol production for $\mathrm{CO}$ hydrogenation due to the favorable thermal and electron conductive properties, as well as defined nanochannel morphology. ${ }^{14}$ However, no other carbonaceous materials have been employed as supports for CO hydrogenation.

It has been well studied that on graphitic carbonaceous materials, alloy surfaces can be created which cannot be produced on refractory oxides. That is, there is always metal segregation instead of alloy phase formation on refractory oxides. Second, on properly prepared carbonaceous materials, it is likely to directly bond the metal to carbon atoms in surface, meaning it can also 
create sintering-resistant metal particles of a unique structure and catalytic potential. ${ }^{13}$ Herein, a structurally ordered, CMK-1 type mesoporous carbon nanoparticles (MCN) was successfully developed by Lin's group ${ }^{15}$ and used to support catalytic particles via surface mediated organometallic reaction. After subsequent functionalization, Rh-Mn/MCN possesses highlyordered tetragonal mesoporous structure as well as MCN and metal catalysts are well-dispersed inside the mesopores. The curved mesoporosity of Rh-Mn/MCN might not only provide a wellconfined environment for metal catalysts but also quickly dissipate the local heating generated from the extremely exothermic $\mathrm{C}_{2+}$ oxygenate formation. Therefore, we also reported the reactivity and selectivity of $\mathrm{CO}$ hydrogenation reaction over $\mathrm{Rh}-\mathrm{Mn} / \mathrm{MCN}$, which was investigated both in micro-tubular reactor and pilot scale fix-bed reactor.

Results and Discussion

Mesoporous carbon nanoparticles $(\mathrm{MCN})$ were first prepared via hard-template synthesis developed in Lin group. The MCN material is consisted of monodisperse (100-200 nm) spherical nanoparticles, possessing a highly ordered mesoporous structures $\left(I 4_{1} / a\right)$ and a narrow distributed pore diameter $(2.3 \mathrm{~nm})$ throughout the entire particle. It has been well known that carbon based materials are highly hydrophobic and chemical treatment or modification on the surface of carbonaceous materials was found to be useful for manipulating their hydrophobic or hydrophilic character. ${ }^{16}$ Furthermore, there is a strong interaction observed between the metals and the functional groups generated by oxidation on the surface of carbonaceous materials. ${ }^{17}$ Among a variety of chemical treatments on carbon based materials, oxidation with nitric acid is the most effective one. Abundant carboxylic acid groups will be created and assist the coordination of metal precursors through the oxygen atoms on the surface of carbon materials. ${ }^{18}$ In order to obtain the highly dispersed $\mathrm{Rh}-\mathrm{Mn}$ nanoparticles, $\mathrm{MCN}$ was carried out in concentrated nitric acid to produce carboxylic acids on the surface (COOH-MCN), followed by 
the deprotonation in sodium carbonate solution (COONa-MCN). Through surface mediated organometallic reaction, ${ }^{19} \mathrm{COONa}-\mathrm{MCN}$ was reacted with $\mathrm{Rh}(\mathrm{I})$ complex in toluene, followed by the addition of manganese nitrate ethanolic solution under nitrogen. The metal complex MCNs were subsequently reduced at $300^{\circ} \mathrm{C}$ under hydrogen gas flow to obtain Rh-Mn/MCN. (Scheme 1. )

In order to scrutinize the catalytic reactivity, we tested the $\mathrm{CO}$ hydrogenation catalyzed by the Rh-Mn/MCN under the pressure of $450 \mathrm{psi}$ at $300^{\circ} \mathrm{C}$ with a total flow rate $33 \mathrm{~mL} / \mathrm{min}$ $\left(\mathrm{H}_{2} / \mathrm{CO}=2 / 1\right)$ in a high-pressure micro-tubular reactor. (Figure 6.) All the gas/liquid products generated from the reaction were analyzed by GC. As shown in task 5 of Table 1., we discovered that no significant methane production was observed in GC analysis. In addition, $\mathrm{Rh}-\mathrm{Mn} / \mathrm{MCN}$ exhibited considerable selectivity toward $\mathrm{C}_{2+}$ oxygenates after reaction for $21 \mathrm{hr}$. Moreover, we also demonstrated the catalytic performance by using pilot-scale fixed bed reactor, which is collaborated with Dr. Brown's group. (Figure. x)

The results from task 1 to task 4 in Table 1 are collected by pilot-scale fixed bed reactor. (describe a bit about exp) Based the results from task 1 to task 3, we discovered that the space time yield (STY) of $\mathrm{C}_{2+}$ oxygenate is highly correlated to the gas hourly space velocity (GHSV). The higher GHSV is, the less the formation of $\mathrm{C}_{2+}$ oxygenates. (Figure. 1a) However, there is no methane production with lower CO conversion in task 3, which was carried out with the highest GHSV. It could be attributed to the short contact between the adsorbents and catalytic metal particles, meaning that there is no huge quantity of dissociative $\mathrm{CO}$ adsorption taking place on metal particles for the formation of active $\mathrm{CH}_{\mathrm{x}}$ species, followed by the $\mathrm{CO}$ insertion to produce $\mathrm{C}_{2+}$ oxygenates. Therefore, task 1 has the highest observed STY of $\mathrm{C}_{2+}$ oxygenates owing to the lowest GHSV. As aforementioned, methane is the most thermodynamically favored product and 
will increase while increasing reaction temperature; nevertheless, the selectivity toward methane production appeared not to increase over $\mathrm{Rh}-\mathrm{Mn} / \mathrm{MCN}$ while the temperature was elevated from $300^{\circ} \mathrm{C}$ to $325^{\circ} \mathrm{C}$. In addition, the $\mathrm{STY}$ of $\mathrm{C}_{2+}$ oxygenates of $\mathrm{Rh}-\mathrm{Mn} / \mathrm{MCN}$ also increases at higher temperature while CO conversion did not vary so much. (Figure. 1b) The change of selectivity could be attributed to the diffusivity of gas and/or products in the meso-pores of carbonaceous materials. ${ }^{20}$ If the diffusion rate is high enough, the heat produced from the extremely exothermic $\mathrm{CO}$ hydrogenation on the active sites might be removed effectively through the well-ordered pores, resulting in prevention of local overheating inside the catalyst. In addition, owing to the hydrophobic nature of carbonaceous materials, the hydrophilic products such as methanol, ethanol and propanol could be easily transported through the catalyst system. To understand more how Rh-Mn/MCN affect the catalytic performance, Rh-Mn/MCN was characterized by a series of different techniques, including transmission electron microscopy (TEM), powder X-ray diffraction (XRD), $\mathrm{N}_{2}$ sorption analysis, and X-ray photoelectron specstroscopy (XPS).

Many research activities have been investigated to understand the influence of the catalyst nature on the catalytic performance. Among those factors, the size and the dispersion of metal particles over the supports are of great importance because CO hydrogenation is a structuresensitive reaction. ${ }^{8,9}$ In general, reactions involving either bond breaking or formation of carboncarbon bond are usually structure sensitive, implying that the formation of supported particles with suitable size should be critical to enhance the catalytic activity and selectivity to $\mathrm{C}_{2}$ oxygenates. ${ }^{21}$ From the reported results, higher $\mathrm{CO}$ conversion and $\mathrm{C}_{2}$-oxygenates selectivity were obtained over $\mathrm{Rh}$ particles between 2-6 nm. ${ }^{9}$ As shown in Figure 1(a),, in spite of successive functinaliztion on the surface of $\mathrm{MCN}$, Rh-Mn/MCN possessed monodisperse spherical particles and highly ordered mesoporous structure without obvious dark spots 
indicating the location of metal particles under low magnification of TEM bright field mode. We assumed the metallic particles are too small to be observed under TEM bright field mode. However, Rh-Mn nanoparticles would be likely to be scoped under STEM (scanning transmission electron microscropy) mode, which is highly sensitive to the discrepancy among light and heavy elements. Consequently, as shown in HAADF-STEM (high angle annular dark field) micrographs (Figure 2(a).), Rh-Mn nanoparticles are able to exhibit stronger contrast than carbon support and are distinguishable from MCN. The size of Rh-Mn nanoparticles is 2-3 nm in diameter and the Rh-Mn nanoparticles are well allocated over MCN. This homogeneous dispersion of Rh-Mn nanoparticles over MCNs is likely to be attributed to the surface mediated organometallic reaction.

After catalytic reaction, it is noteworthy of the growth of $\mathrm{Rh}-\mathrm{Mn}$ nanoparticles as shown in Figure 2(b).. The size of Rh-Mn nanoparticles before syngas reaction was 2-3 nm large in diameter and some of them increased up to $6 \mathrm{~nm}$ after reaction (Figure 2(b).) owing to the thermal sintering along with high temperature reaction. However, most of the nanoparticles have maintained the size $2-3 \mathrm{~nm}$, which is about the size of the pore diameter. (Figure 2(a) and 2(b).) Although some metal particles have grown larger than the pore size, neither the TEM images nor BET isotherms (shown below) provide evidence for destruction of the porous structure of MCN. After the sample was ultramicrotomed into $60 \mathrm{~nm}$ in thickness, the distribution of $\mathrm{Rh}-\mathrm{Mn}$ nanoparticle inside the mesopores was illustrated in Figure 1(c).. It was found that those metal particles were highly dispersed over MCN. Herein, it appears that the growth of most Rh-Mn nanoparticles is confined by the pore diameter, which is consistent with the conclusion reported in literature. ${ }^{9,22}$ They have discovered that the catalytic particles are uniformly deposited inside the support pores and limited by the pore size. To our knowledge, thermal sintering processes 
involve either migration of adatoms of metal (Ostwald ripening) or migration and coalescence of metal nanoparticles. Metal nanoparticles of the size range investigated here are quite mobile even at room temperature. ${ }^{23}$ Therefore, it comes out that the circuitous pore arrangements within the spherical MCN assisted to restrict the mobility of Rh-Mn nanoparticles and prevent them from a high degree of sintering. ${ }^{23}$

In order to confirm that the porous structure of $\mathrm{Rh}-\mathrm{Mn} / \mathrm{MCN}$ is unlikely to be damaged after consecutive surface functionalization and is well perserved after catalytic reactions, the nitrogen sorption properties of $\mathrm{Rh}-\mathrm{Mn} / \mathrm{MCN}(\mathrm{rxn})$ (after catalytic reaction) as well as $\mathrm{Rh}-\mathrm{Mn} / \mathrm{MCN}$, $\mathrm{COOH}-\mathrm{MCN}$ and MCN were analyzed. As summarized in Table 2., the total surface areas and the average pore diameters were determined by the Brunauer- Emmett-Teller (BET) and Barrett-Joyner-Halenda (BJH) methods, respectively. The BET isotherms of these four materials exhibited the characteristic Type IV adsorption/desorption pattern, indicating the presence of highly ordered mesoporous structure. (Figure 3.) In addition, Rh-Mn/MCN still exhibited high surface area $\left(>1400 \mathrm{~cm}^{2} \mathrm{~g}^{-1}\right)$ and high pore volume $\left(>0.8 \mathrm{~cm}^{3} \mathrm{~g}^{-1}\right)$. Nevertheless, it is worth mentioning that the surface area of $\mathrm{Rh}-\mathrm{Mn} / \mathrm{MCN}(\mathrm{rxn})$ decreased as shown above in Figure 3.. Rh-Mn nanoparticles would grow up as a result of thermal sintering and were likely to be restricted by the porous structure of MCN, meaning that the size of Rh-Mn nanoparticles is likely to be limited as large as pore diameter. Therefore, the decrease of surface area and total pore volume could be attributed to the partially impeded pore accessibility by Rh-Mn nanoparticles. In addition, Rh-Mn/MCN(rxn) possessed narrow pore size distribution as well as Rh-Mn/MCN, indicating that catalytic sites were mostly distributed inside the pores.

Figure 4. demonstrated power X-ray diffraction patterns of $\mathrm{MCN}, \mathrm{COOH}-\mathrm{MCN}$, RhMn/MCN and Rh-Mn/MCN(rxn). (top to bottom, respectively) After oxidation with nitric acid, 
COOH-MCN still possessed three characteristic peaks (110), (211) and (220) of CMK-1 as same as $\mathrm{MCN}$, indicating that carboxylic acid functionality did not alter the pore symmetry of MCN after oxidation. On the other hand, the intensities of Rh-Mn/MCN and Rh-Mn/MCN(rxn) decreased dramatically after the immobilization of $\mathrm{Rh}-\mathrm{Mn}$ nanoparticles and syngas reaction. The decrease could be attributed to the partial pore-filling effect caused by the growth of Rh-Mn nanoparticles, which is also consistent with the results of TEM and nitrogen sorption.

In order to learn the correlation between metal nanoparticles and the reactivity/selectivity of CO hydrogenation, XPS (X-ray Photoelectron Spectroscopy) was employed to determine the oxidation states of $\mathrm{Rh}$ and $\mathrm{Mn}$ before and after hydrogen reduction, which are shown in Table $\mathrm{x}$ and Figure $\mathrm{x}$. According to the XPS analysis, we realized the greater binding energy of the component, the higher oxidation state. It can be found that the binding energy of the Rh3 $d_{5 / 2}$ of $\mathrm{Rh}-\mathrm{Mn} / \mathrm{MCN}$ before hydrogen reduction is $307.0 \mathrm{eV}$, less than the one after reduction (308.3 $\mathrm{eV}$ ), which is consistent with the reported value of $\mathrm{Rh}^{0.24}$ This indicats that pretreatment is sufficient and necessary to reduce easily-oxidized $\mathrm{Rh}$ complex into $\mathrm{Rh}^{0}$ and $\mathrm{Rh}$ mainly existed in the metallic state $\left(\mathrm{Rh}^{0}\right)$ on the carbon surface during $\mathrm{CO}$ hydrogenation. In other words, $\mathrm{Rh}^{0}$ is active for the formation of $\mathrm{C}_{2+}$ oxygenates. However, $\mathrm{Rh}^{+}$might exist during the reaction owing to an unstable intermediate derived from $\mathrm{Rh}^{0}$ after oxidation by oxygen atom dissociated from $\mathrm{CO}$ molecules. The role of $\mathrm{Rh}^{+}$playing in $\mathrm{CO}$ hydrogenation has been well studied ${ }^{25,26}$ and it is well known as well that chemisorbed-CO on $\mathrm{Rh}^{0}$ at room temperature would lead isolated $\mathrm{Rh}^{+}$ to changing into $\mathrm{Rh}^{0}$ after $\mathrm{CO}$ desorption. Therefore, there should be only negligible amounts of $\mathrm{Rh}^{+}$on the supportted catalyst.

For the oxidation state of $\mathrm{Mn}$ in $\mathrm{Rh}-\mathrm{Mn} / \mathrm{MCN}$, it was determined that the binding energies of Mn species were close $(641.0 \mathrm{eV}$ and $641.8 \mathrm{eV})$, indicating that $\mathrm{Mn}$ existed in the state of $\mathrm{Mn}^{2+}$ 
or higher valence states $\left(\mathrm{Mn}^{3+}\right.$ and $\left.\mathrm{Mn}^{4+}\right)$ before and after reduction. ${ }^{24} \mathrm{Mn}^{2+}$ was unlikely to be reduced to metallic state and could influence the selectivity effectively with its high valence states as a promotor during $\mathrm{CO}$ hydrogenation.

Conclusion

In conclusion, ordered mesoporous carbon nanoparticles (MCN) have been successfully demonstrated as a catalytic support for $\mathrm{CO}$ hydrogenation and exhibited interesting effect on catalytic performance. Owing to the unique mesoporosity, catalytic metal particles were highly distributed over $\mathrm{MCN}$ and $\mathrm{Rh}-\mathrm{Mn} / \mathrm{MCN}$ might be able to suppress methane production at higher temperature. In order to study the impact of mesostructures, the reactivity and selectivity of $\mathrm{CO}$ hydrogenation on Rh-supported mesoporous carbon materials with different porous structures such as CMK-3, CMK-5 and CMK-8 are currently under investigation.

Experimental

Preparations of Materials

Preparation of aluminosilicate MCM-48: For synthesis of OMC, the silica was converted to an aluminosilicate form using a solution of $\mathrm{AlCl}_{3}$ following the postsynthesis procedure: as-synthesized MCM-48 material was calcined at $550{ }^{\circ} \mathrm{C}$ to remove the surfactant. The calcined sample was mixed with distill water to make surface silanol groups, and then completely dried at $150{ }^{\circ} \mathrm{C}$. The dried sample was slurried in ethanol solution of the anhydrous $\mathrm{AlCl}_{3}(\mathrm{Si} / \mathrm{Al}=20)$. The ethanol solvent was completely evaporated by rotary evaporator. The dried sample was calcined again at $550{ }^{\circ} \mathrm{C}$.

Preparation of MCN: Ordered mesoporous carbon nanoparticles MCNs were prepared using furfuryl alcohol (Aldrich) as a carbon source. 1g of aluminated MCM-48 nanoparticles were infiltrated with $0.91 \mathrm{~mL}$ of furfuryl alcohol by impregnation method. The mixture was moved into Schlenk reactor, and subjected to freeze-vacuum-thaw three times using liquid $\mathrm{N}_{2}$. The 
mixture was kept under vacuum at $35^{\circ} \mathrm{C}$ for $1 \mathrm{~h}$. After opened schlenk reactor, the mixture was heated for $6 \mathrm{~h}$ at $100{ }^{\circ} \mathrm{C}$ for polymerization of furfuryl alcohol, and then partially carbonized at $350{ }^{\circ} \mathrm{C}$ for $3 \mathrm{~h}$ under vacuum. After cooled to room temperature, the sample was added with 0.58 $\mathrm{mL}$ of furfuryl alcohol, and repeated freeze-vacuum-thaw and polymerization again. Further carbonization was accomplished by heating to $900{ }^{\circ} \mathrm{C}$ under vacuum condition. The carbon product was collected by $\mathrm{HF}$ washing. (10 wt $\% \mathrm{HF}$ in $\mathrm{EtOH} / \mathrm{H}_{2} \mathrm{O}$ solution $)^{15}$

Preparation of $\mathrm{COOH}-\mathrm{MCN}$ : $300 \mathrm{mg}$ of $\mathrm{MCNs}$ were placed in a $100 \mathrm{~mL}$ round-bottom flask with $30 \mathrm{~mL}$ nitric acid $(1 \mathrm{M})$ and the solution was refluxed at $110^{\circ} \mathrm{C}$ for $1.5 \mathrm{~h}$, then followed by hot filtration and washed with copious of water till the $\mathrm{pH}$ value of aqueous filtrate reached 7. The as-synthesized carbon material was dried under vacuum at $100^{\circ} \mathrm{C}$ for $8 \mathrm{~h}$ before further usage.

Preparation of COONa-MCN: 150mg of $\mathrm{COOH}-\mathrm{MCN}$ sere mixed with sodium carbonate aqueous solution $\left(80 \mathrm{mg} \mathrm{Na}_{2} \mathrm{CO}_{3} / 20 \mathrm{~mL} \mathrm{H}_{2} \mathrm{O}\right.$ ) and the solution was stirred at $30^{\circ} \mathrm{C}$ for $6 \mathrm{~h}$, followed by filtration and washed with considerable amount of water. Likewise, the assynthesized carbon material was dried under vacuum at $100^{\circ} \mathrm{C}$ for $8 \mathrm{~h}$ before further usage.

Preparation of Rh-Mn/MCN: 148.3mg of COONa-MCNs were placed in 50mL double-neck round-bottom flask and mixed with $2.8 \mathrm{mg}$ of $\mathrm{Rh}_{2} \mathrm{Cl}_{2} \mathrm{CO}_{4}(2 \mathrm{wt} \%$ of $\mathrm{Rh}$ loading over $\mathrm{Rh}$ $\mathrm{Mn} / \mathrm{MCN}$ ) in $5 \mathrm{~mL}$ toluene under nitrogen. The solution was stirred at $60^{\circ} \mathrm{C}$ for $3 \mathrm{~h}$. After three hour reaction, the prepared $9.6 \mathrm{mg} \mathrm{Mn}\left(\mathrm{NO}_{3}\right)_{2} / 5 \mathrm{~mL}$ ethanol solution (2 wt \% $\mathrm{Mn}$ loading over $\mathrm{Rh}$ $\mathrm{Mn} / \mathrm{MCN}$ ) was added in the flask with three further hour reaction at $60^{\circ} \mathrm{C}$. The metal complex MCNs were filtered, washed with hexane and dried under vacuum overnight. In order to obtain Rh-Mn/MCNs, the metal complex MCNs were reduced under hydrogen with a gas flow rate $(1.67 \mathrm{~mL} / \mathrm{s})$ at $300^{\circ} \mathrm{C}$ for $3 \mathrm{~h}$ with heating $\operatorname{ramp}\left(2^{\circ} \mathrm{C} / \mathrm{min}\right)$. The as-synthesized $\mathrm{Rh}-\mathrm{Mn} / \mathrm{MCN}$ was 
washed with water to remove $\mathrm{NaCl}$ then dried under vacuum at $100^{\circ} \mathrm{C}$ for $8 \mathrm{~h}$ before the catalytic reaction. $^{19}$

\section{Preparation of Catalytic Reaction}

$\mathrm{Rh}-\mathrm{Mn} / \mathrm{MCN}$ (0.3g) was placed in a micro-tubular reactor to examine $\mathrm{CO}$ hydrogenation under the pressure of $450 \mathrm{psi}$ at $300^{\circ} \mathrm{C}$ for $21 \mathrm{~h}$ with syngas of $\mathrm{H}_{2} / \mathrm{CO}=2 / 1$ and a total flow 33 $\mathrm{mL} / \mathrm{min}$. $\left(\mathrm{GHSV}=6600 \mathrm{~h}^{-1}\right)$ Prior to reaction, the catalyst was reduced at $300^{\circ} \mathrm{C}$ in a flow of $\mathrm{H}_{2}$ (10 $\mathrm{mL} / \mathrm{min}$ ) under 450psi for $2 \mathrm{~h}$. The effluent flew through a condenser filled with $10 \mathrm{~mL}$ nano-pure water. The tail gas was analyzed on-line by Varian GC (CP 3900) equipped with a packed column and a TCD detector. The dissolved oxygenate products were analyzed off-line by the GC (CP 3900) equipped with a CP-PoraBOND Q Fuse Silica capillary column and a FID detector.

General Characterization

XRD patterns were recorded on a Scintag XDS-2000 instrument operated at $1.21 \mathrm{~kW}$, using $\mathrm{Cu} \mathrm{K \alpha}$ radiation. The nitrogen adsorption isotherms were measured at liquid nitrogen temperature (77 K) using a Micromeritics ASAP2000 volumetric adsorption analyzer. The Brunauer-Emmett-Teller (BET) equation was used to calculate the apparent surface area from adsorption data obtained at $\mathrm{P} / \mathrm{P}_{0}$ between 0.05 and 0.2 . The total volume of micro- and mesopores was calculated from the amount of nitrogen adsorbed at $\mathrm{P} / \mathrm{P}_{0}=0.95$, assuming that adsorption on the external surface was negligible compared to adsorption in pores. The pore size distributions (PSD) were calculated by analyzing the adsorption branch of the $\mathrm{N}_{2}$ sorption isotherm using the Barret-Joyner-Halenda (BJH) method. SEM (scanning electron microscopy) images were obtained with a JEOL $840 \mathrm{~A}$ scanning electron microscope operating at $10 \mathrm{kV}$. The samples were coated with gold before SEM measurement. TEM (transmission electron microscopy) images were taken from particles supported on a porous carbon grid, using a Tecnai 
G2 F20 equipment operated at $200 \mathrm{kV}$. The catalytic performance was carried out in a microtubular reactor and the tail gas was analyzed on-line by Varian GC (CP 3900) equipped with a packed column and a TCD detector. The dissolved oxygenate products were analyzed off-line by the GC (CP 3900) equipped with a CP-PoraBOND Q Fuse Silica capillary column and a FID detector with hydrogen as carrier gas, and 1-butanol as internal standard. XPS (X-ray photoelectron spectroscopy) measurements of the samples were completed on a PHI 5500 Physical Electronic Multitechnique system. The testing conditions were as follows: radiation source, Al standard X-ray source $(\mathrm{Al} K \alpha$ radiation, $\mathrm{hv}=1486.6 \mathrm{eV}), \mathrm{PE}=58.7 \mathrm{eV}$; detection area $=1.5 \mathrm{~mm}^{2}$, pressure in the analysis chamber $=10^{-9}$ Torr. Binding energies (BEs) were determined with an accuracy of $\pm 0.1 \mathrm{eV}$. The charging effects were measured against adventitious carbon of C1s $(284.8 \mathrm{eV})$.

Scheme 1. Synthesis of Rh-Mn/MCN

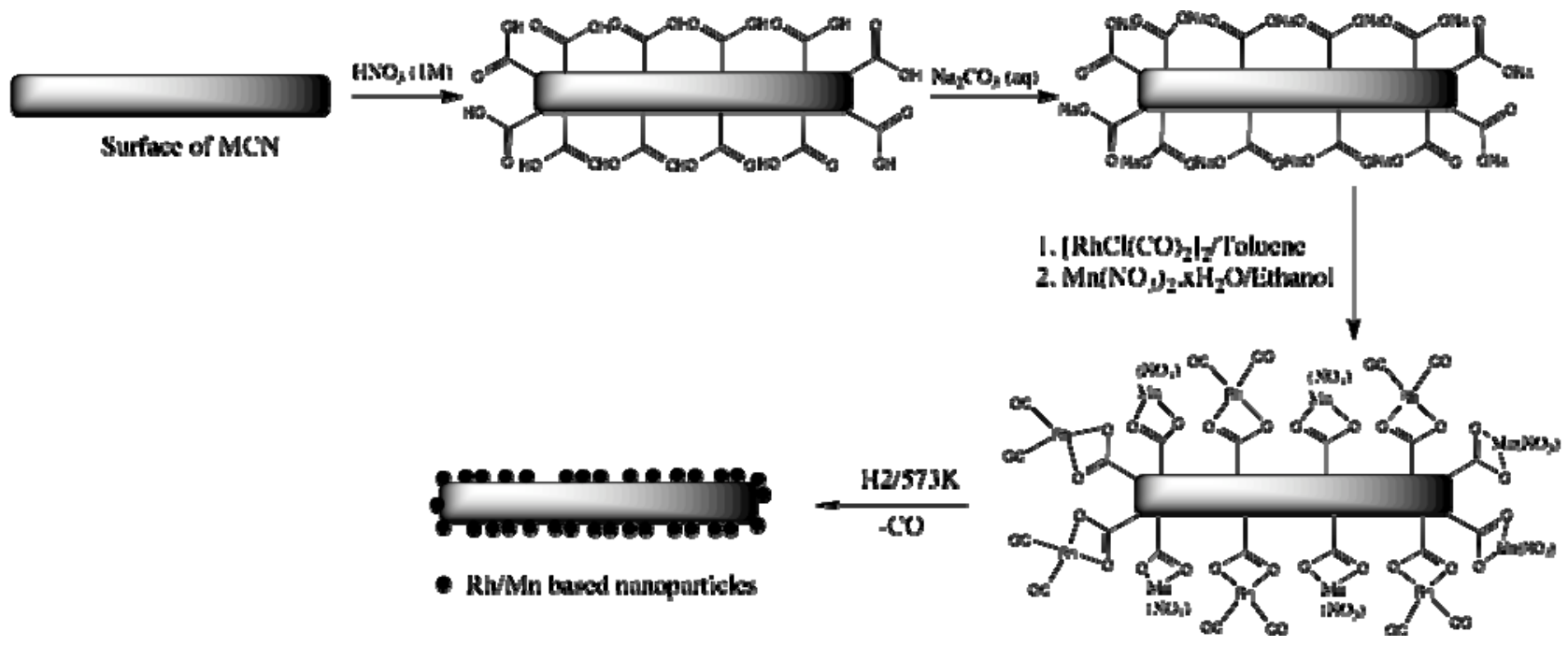




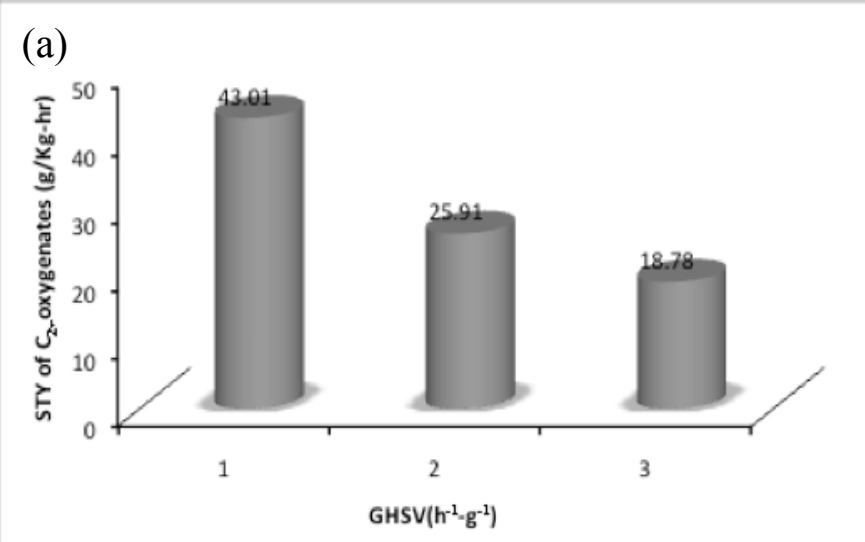

(b)

$=5 \mathrm{TY}$ of $\mathrm{C2}+$ oxygenates $(\mathrm{g} / \mathrm{Kg} \cdot \mathrm{h})$

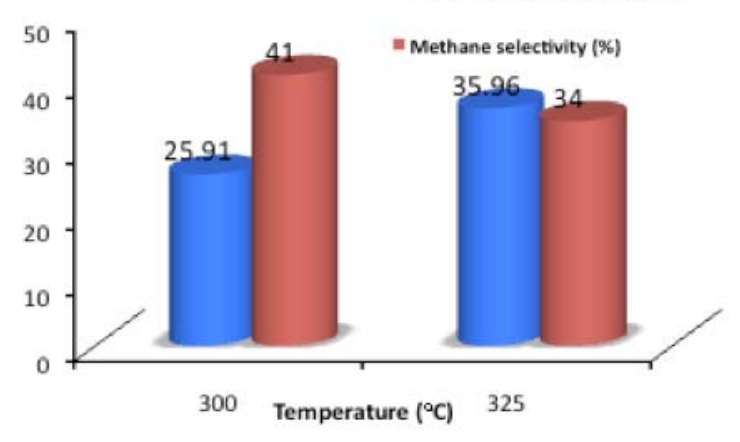

Figure 1. (a) The correlation between GHSV and STY of C2+ oxygenates; (b) The comparison of methane selectivity and STY of C2+ oxygenates
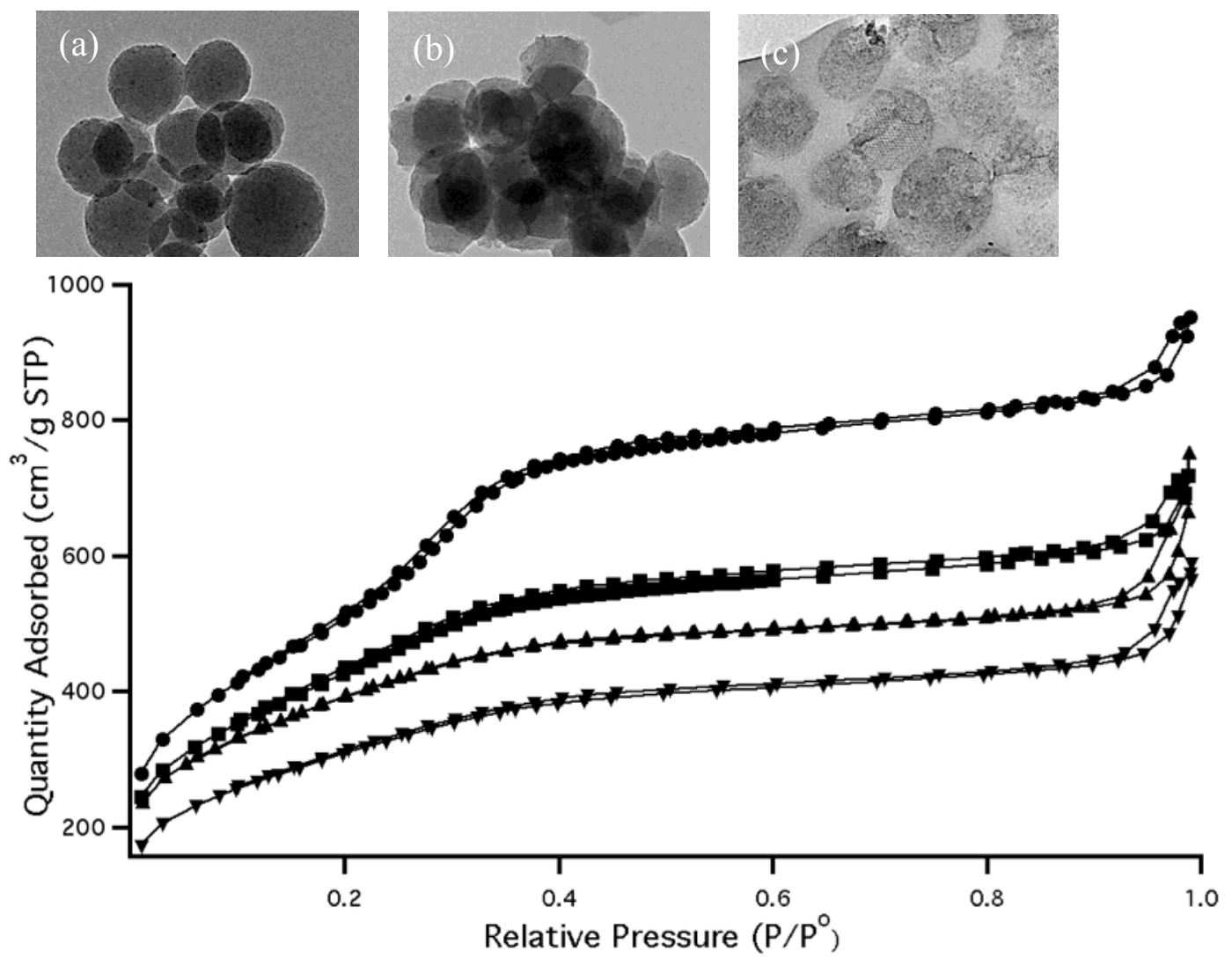

l) (b);

Figure 3. BET isotherms of surface functionalized $\mathrm{MCN}$, - - $\mathrm{MCN}$; - $\mathbf{-}-\mathrm{COOH}-$ MCN; - $\boldsymbol{\Delta}$ - Rh-Mn/MCN; - $\boldsymbol{\nabla}$ - Rh-Mn/MCN (rxn) 


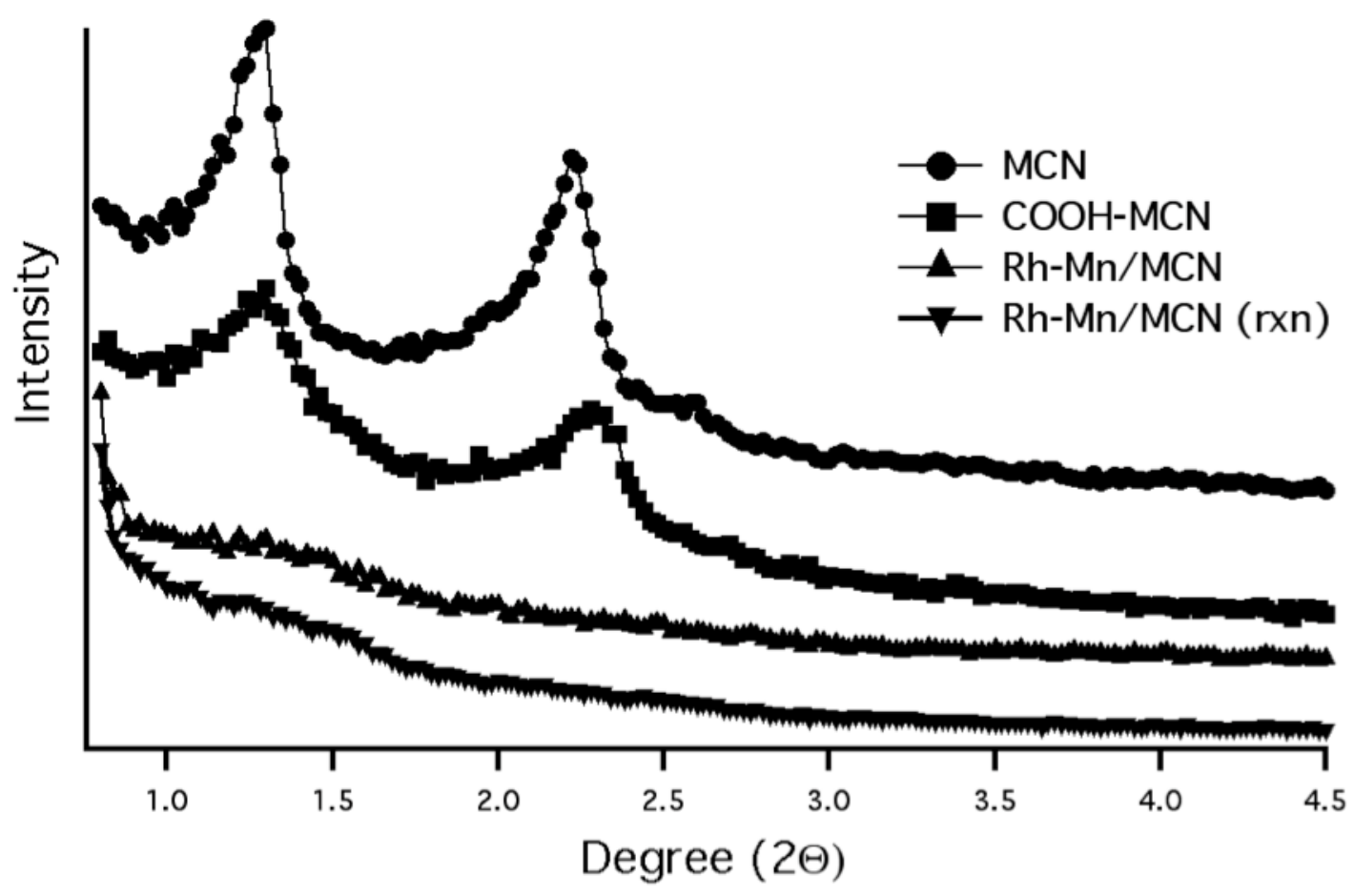

Figure 4. Powder X-ray patterns of surface-functionalized MCN and Rh$\mathrm{Mn} / \mathrm{MCN}$ after reaction)

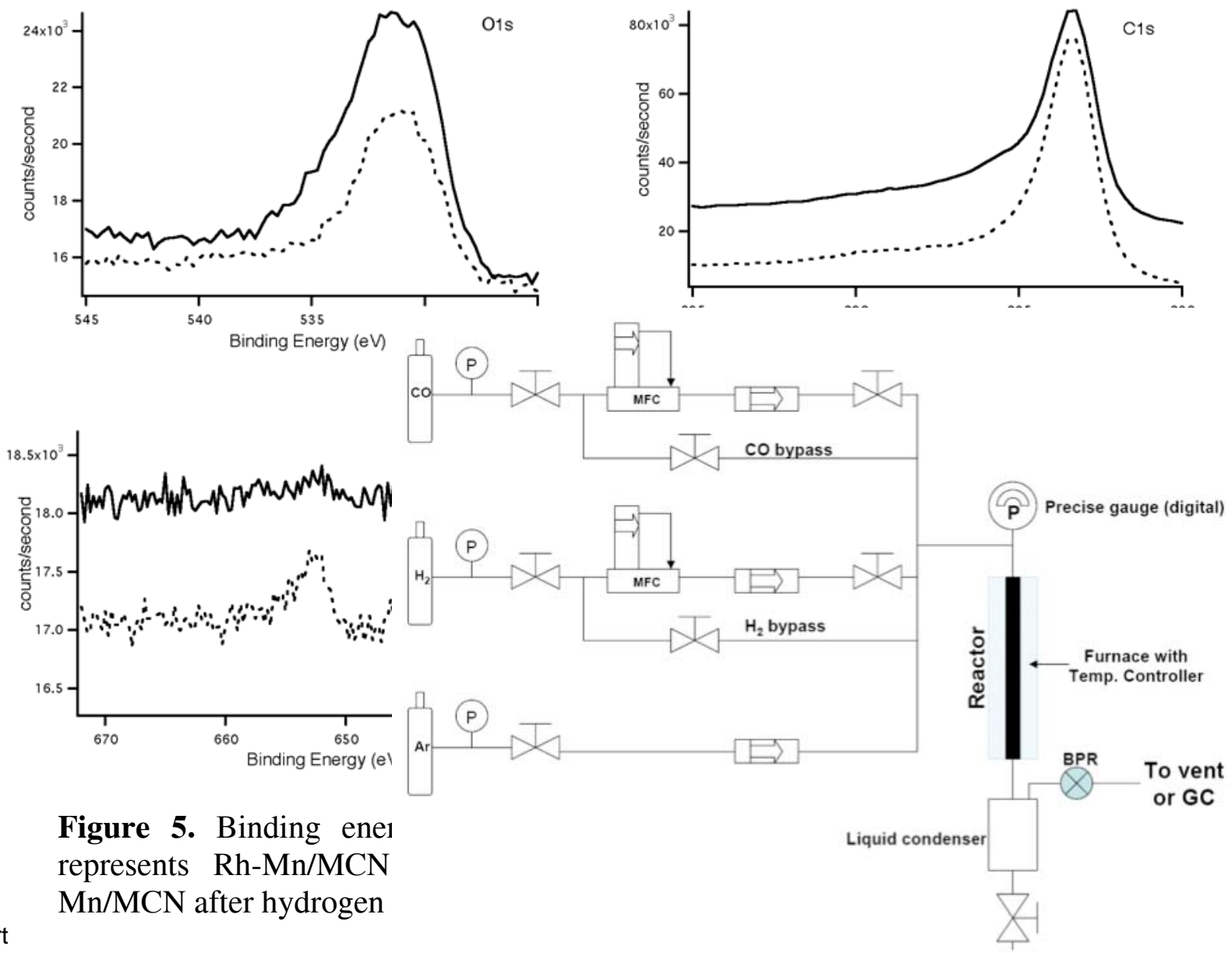

Figure 6. Flow diagram of a micro-tubular reactor 


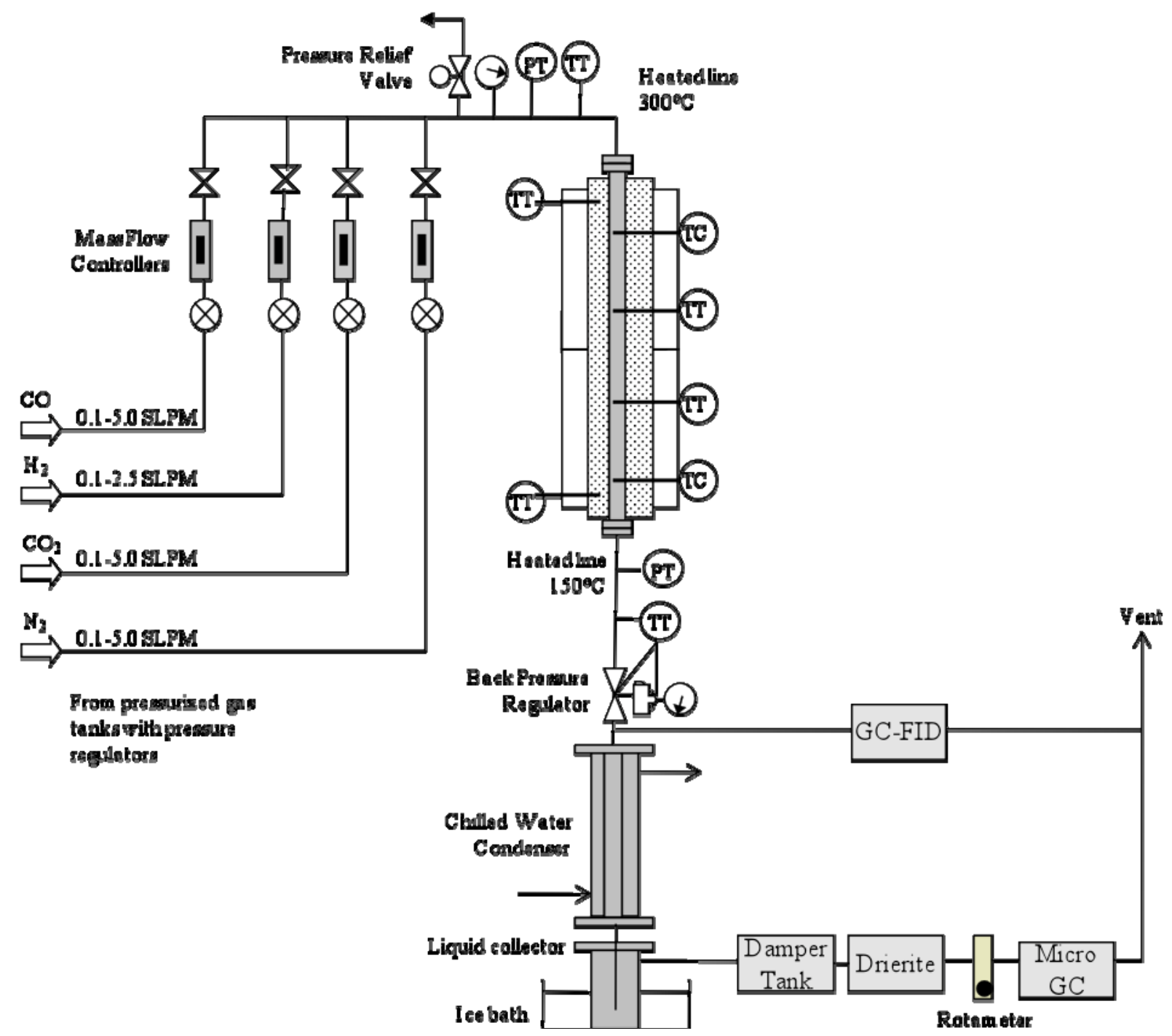

Figure 7. Schematic Diagram of the Fixed-Bed Reactor Setup 
Table 1. The catalytic performance and the selectivity of products

\begin{tabular}{|c|c|c|c|c|c|c|c|c|c|}
\hline \multirow[b]{2}{*}{ Task } & \multirow{2}{*}{$\begin{array}{c}\text { Temperature } \\
\text { Table 3. Bi } \\
\left({ }^{\circ} \mathrm{C}\right)\end{array}$} & GHSV & $\mathrm{CO}$ & \multicolumn{5}{|c|}{ Selectivity (C \%) } & \multirow{2}{*}{$\begin{array}{c}\mathrm{STY}_{\mathrm{Y}} \text { of } \mathrm{C}_{2+} \\
\text { oxygenates } \\
(\mathrm{g} / \mathrm{kg}-\mathrm{hr})\end{array}$} \\
\hline & & $\begin{array}{c}\text { dinglene } \\
1)\end{array}$ & $\begin{array}{l}\text { onyersi- } \\
\text { s\%) }\end{array}$ & $\begin{array}{l}\mathrm{MCN} \\
\mathrm{CH}_{4}\end{array}$ & $\mathrm{C}_{2+} \mathrm{HC}$ & $\begin{array}{l}\text { fter red } \\
\mathrm{CO}_{2}\end{array}$ & MeOH & $\begin{array}{c}\mathrm{C}_{2+} \\
\text { oxygenates }\end{array}$ & \\
\hline 1 & 300 & 9,300 & N/A & N/A & N/A & N/A & N/A & N/A & 43.01 \\
\hline 2 & 300 & 18,600 & 18.00 & 41.00 & 7.00 & 26.00 & 11.76 & 14.24 & 25.91 \\
\hline 3 & 300 & 37,200 & 5.00 & 0 & 9.00 & 14.00 & 42.80 & 34.20 & 18.78 \\
\hline 4 & 325 & 18,600 & 17.00 & 34.00 & 8.00 & 20.00 & 20.80 & 17.20 & 35.96 \\
\hline 5 & 300 & 6,607 & 3.27 & 36.97 & 0 & 20.31 & 19.69 & 28.04 & 27.16 \\
\hline
\end{tabular}

N/A: Accurate gas composition is not able to be obtained due to the low flow rate.

Table 2. Structural properties of surface-functionalized $\mathrm{MCN}^{\mathrm{a}}$

\begin{tabular}{llll}
\hline Samples & $\begin{array}{l}S_{\mathrm{BET}} \\
\left(\mathrm{m}^{2} \mathrm{~g}^{-1}\right)\end{array}$ & $\begin{array}{l}W_{\mathrm{BJH}} \\
(\mathrm{nm})\end{array}$ & $\begin{array}{l}V_{\mathrm{p}} \\
\left(\mathrm{cm}^{3} \mathrm{~g}^{-1}\right)\end{array}$ \\
\hline MCN & 1874.3 & 2.9 & 1.32 \\
$\mathrm{COOH}-\mathrm{MCN}$ & 1567.2 & 2.3 & 0.97 \\
$\mathrm{Rh}-\mathrm{Mn} / \mathrm{MCN}$ & 1419.6 & $<1.8$ & 0.84 \\
$\mathrm{Rh}-\mathrm{Mn} / \mathrm{MCN}$ & 1131.7 & $<1.8$ & 0.71 \\
$($ after reaction $)$ & & & \\
\hline${ }^{\mathrm{a}}: S_{\mathrm{BET}}$ stand for surface area; $W_{\mathrm{BJH}}$ stand for pore diameter; $V_{\mathrm{p}}$ stand for total pore voume \\
\hline
\end{tabular}




\begin{tabular}{cccc}
\hline \multirow{2}{*}{ Sample } & Testing conditions & \multicolumn{2}{c}{ Binding energies $(\mathrm{eV})$} \\
\cline { 3 - 4 } Rh-Mn/MCN & $\mathrm{Mn} 2 p_{3 / 2}$ & $\mathrm{Rh}^{\mathrm{a}} 3 d_{5 / 2}$ \\
& Before reduction & 641.0 & 308.3 \\
& After reduction & 641.8 & 307.0 \\
\hline
\end{tabular}

a: In order to obtain higher resolution of XPS, the sample was prepared with higher metal loading (4 wt \% Rh-4 wt\% Mn/MCN)

References

(1) George, H.; Avelino, C. Angew. Chem. Int. Ed. 2007, 46, 7184.

(2) Spivey, J. J.; Egbebi, A. Chem. Soc. Rev. 2007, 36, 1514.

(3) Subramani, V.; Gangwal, S. K. Energy \& Fuels 2008, 22, 814.

(4) Ichikawa, M.; Fukushima, T. J. Chem. Soc., Chem. Commun. 1985, 321.

(5) Reuel, R. C.; Bartholomew, C. H. J. Catal. 1984, 85, 78.

(6) Vannice, M. A. J. Catal. 1975, 37, 449.

(7) Murchison, C. B.; Murdick, D. A. Hydrocarb. Process. 1981, 60, 159.

(8) Tago, T.; Hanaoka, T.; Dhupatemiya, P.; Hayashi, H.; Kishida, M.; Wakabayashi, K. Catal. Lett. 2000, 64, 27.

(9) Zhou, S. T.; Zhao, H.; Ma, D.; Miao, S. J.; Cheng, M. J.; Bao, X. H. Z. Phys. Chemie-Int. J. Res. Phys. Chem. Chem. Phys. 2005, 219, 949.

(10) Gao, J.; Mo, X.; Chien, A. C.-Y.; Torres, W.; Goodwin Jr, J. G. J. Catal. 2009, 262, 119.

(11) Chuang, S. S. C.; Stevens, R. W.; Khatri, R. Top. Catal. 2005, 32, 225.

(12) Arakawa, H.; Takeuchi, K.; Matsuzaki, T.; Sugi, Y. Chem. Lett. 1984, 1607.

(13) Phillips, J.; Weigle, J.; Herskowitz, M.; Kogan, S. Applied Catalysis A: General 1998, $173,273$.

(14) Pan, X.; Fan, Z.; Chen, W.; Ding, Y.; Luo, H.; Bao, X. Nature Materials 2007, 6, 507.

(15) Kim, T.-W.; Chung, P.-W.; Slowing, I. I.; Tsunoda, M.; Yeung, E. S.; Lin, V. S. Y. Nano Lett. 2008, 8, 3724.

(16) Rodriguez, N. M. J. Mater. Res. 1993, 8, 3233.

(17) Banerjee, S.; Wong, S. S. Nano Lett. 2002, 2, 49.

(18) Bazula, P. A.; Lu, A.-H.; Nitz, J.-J.; Sch h, F. Microporous Mesoporous Mater. 2008, $108,266$.

(19) Giordano, R.; Serp, P.; Kalck, P.; Kihn, Y.; Schreiber, J.; Marhic, C.; Duvail, J.-L. Eur. J. Inorg. Chem. 2003, 2003, 610.

(20) Okabe, K.; Murata, K.; Mohammad, N.; Liu, Y.; Takahara, I.; Inaba, M. Journal of the Japan Petroleum Institute 2007, 50, 349.

(21) Chen, W. M.; Ding, Y. J.; Jiang, D. H.; Pan, Z. D.; Luo, H. Y. Catal. Lett. 2005, 104, 177.

(22) Hamada, H.; Funaki, R.; Kuwahara, Y.; Kintaichi, Y.; Wakabayashi, K.; Ito, T. Applied Catalysis 1987, 30, 177.

(23) Bore, M. T.; Pham, H. N.; Ward, T. L.; Datye, A. K. Chem. Commun. 2004, 2620.

(24) Wang, Y.; Luo, H.; Liang, D.; Bao, X. J. Catal. 2000, 196, 46. 
(25) Watson, P. R.; Somorjai, G. A. J. Catal. 1981, 72, 347.

(26) Watson, P. R.; Somorjai, G. A. J. Catal. 1982, 74, 282. 


\section{APPENDIX 2}

\section{A New Mesoporous Oxide-Supported Metal Catalyst System for Conversion of Synthetic Gas to Alcohols}

The Intellectual Property Disclosure and Record (IPDR) provides information that the Office of Intellectual Property and Technology Transfer (OIPTT) and the Iowa State University Research Foundation, Inc. (ISURF) need to evaluate your invention/creation. The form is also used as the Ames Laboratory Record of Invention.

It is important that you provide the most comprehensive and complete disclosure possible so that OIPTT/ISURF can make an informed decision on how to proceed with your disclosure. The more complete the disclosure, the more quickly we can provide a response to you. Please complete the form to the best of your ability.

\section{Instructions for completing the IPDR:}

- Text fields will expand, so use as many lines as you need to adequately answer the question.

- If you have prepared documents that answer a question, you may reference those and attach copies. It will be very helpful if you can provide attachments in electronic form.

- For check boxes, double click to fill in the box.

If you have any questions while completing the form, please contact Lynne Mumm, Disclosure Manager, at 515/294-4742 or 1mumm@iastate.edu; or Patsy Duncan, Disclosure Secretary, at 515/294-3113 or pduncan@iastate.edu. 


\section{Submitting the IPDR:}

Ames Lab Employees: Submit the completed IPDR and any attachments to Todd Zdorkowski, Ames Laboratory, Office of Industrial Outreach and Technology Administration, 311 TASF, Ames, IA 50011; direct phone: 515/294-5640. He will then forward the disclosure to Lynne Mumm at ISURF.

Other ISU Employees: Submit the completed IPDR and any attachments to Lynne Mumm, ISURF, 310 Lab of Mechanics Ames, IA 50011-2131; FAX: 515/294-0778;

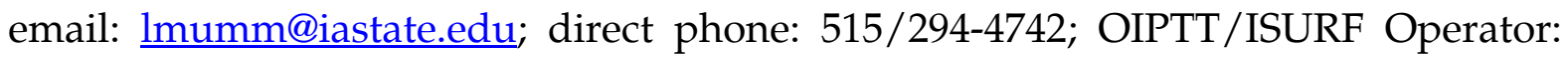
$515 / 294-4740$.

Note: All inventor(s)/creator(s)/author(s) must sign the IPDR. If it is submitted electronically, please provide a hard copy once all ISU signatures have been obtained. If you are having problems obtaining a signature, contact Lynne Mumm for assistance.

Additional information is available in the ISU Intellectual Property Handbook located at http://www.iastate.edu/ isurf/handbook/handbook.html and on our web site located at http://www.iastate.edu/ isurf. 


\section{A. Title of Work:}

A New Mesoporous Oxide-Supported Metal Catalyst System for Conversion of Synthetic Gas to Alcohols

Is this technology related to any invention/creation previously disclosed to ISURF? If yes, please provide additional information below, including ISURF number (if known).

B. Inventor(s)/Creator(s)/Author(s) ("I/C/As"): This does not include individuals that solely provided funding, research materials, equipment, assistance in routine statistical analyses, assistance in routine testing, and standard protocols, or individuals that bring to one's attention the existence of a problem but not the solution. Contact Lynne Mumm (294-4742) if you need assistance in determining the $\mathrm{I} / \mathrm{C} / \mathrm{As}$.

Please be advised that inventorship is a legal determination that will be made at the appropriate time(s) by patent counsel. Although there is no legal significance to the order of names on a patent application or an issued patent, you may consult with the others who are purported to be inventors, and submit their names in the order you would prefer them to appear on a patent application, if one is filed. By signing the IPDR, all purported inventors are agreeing to this order.

\begin{tabular}{|l|l|}
\hline$\bigotimes$ Dr. $\square$ Mr. $\square$ Ms. $\square$ Other & Citizenship: Taiwan \\
\hline Full Name: Victor Shang-Yi Lin & Social Security No.: 190-72-2344 \\
\hline Dept/Affiliation: Chemistry & Home Address: 3227 Bayberry Road \\
\hline Office Address: 0755 Gilman Hall & Home Phone: 515-292-6498 \\
\hline Office Phone: 515-294-3135 & Home email: NA \\
\hline Office Fax: 515-294-0105 & ISUB $\$ \\
\hline Office email: vsylin@iastate.edu Non-ISU $\square$ \\
\hline Inventor? $\bigotimes \quad$ Creator? $\square \quad$ Author? $\square$ \\
\hline Briefly describe contribution: Originated the idea. Principle inventor. \\
\hline
\end{tabular}

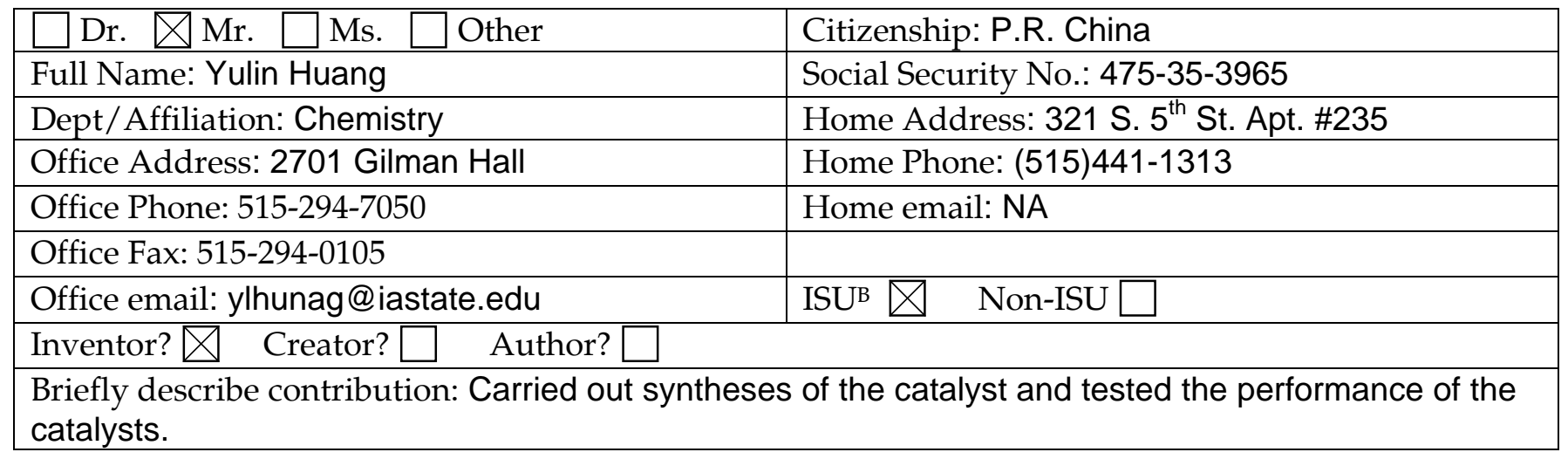

\begin{tabular}{|l|l|}
\hline$\bigotimes$ Dr. $\square$ Mr. $\square$ Ms. $\square$ Other & Citizenship: P.R. China \\
\hline Full Name: Yang Cai & Social Security No.: 484-29-8621 \\
\hline Dept/Affiliation: Chemistry & Home Address: 4620 Webster St., Ames, IA, \\
\hline
\end{tabular}




\begin{tabular}{|l|l|}
\hline & 50014 \\
\hline Office Address: 2710 Gilman Hall & Home Phone: (515)-292-3596 \\
\hline Office Phone: 515-294-2090 & Home email: NA \\
\hline Office Fax: 515-294-0105 & ISU' $\$ \\
\hline Office email: yangcai@iastate.edu Non-ISU $\square$ \\
\hline Inventor? $\square \quad$ Creator? $\square \quad$ Author? $\square$ \\
\hline $\begin{array}{l}\text { Briefly describe contribution: Carried out syntheses of the catalyst and tested the performance of the } \\
\text { catalysts. }\end{array}$ \\
\hline
\end{tabular}

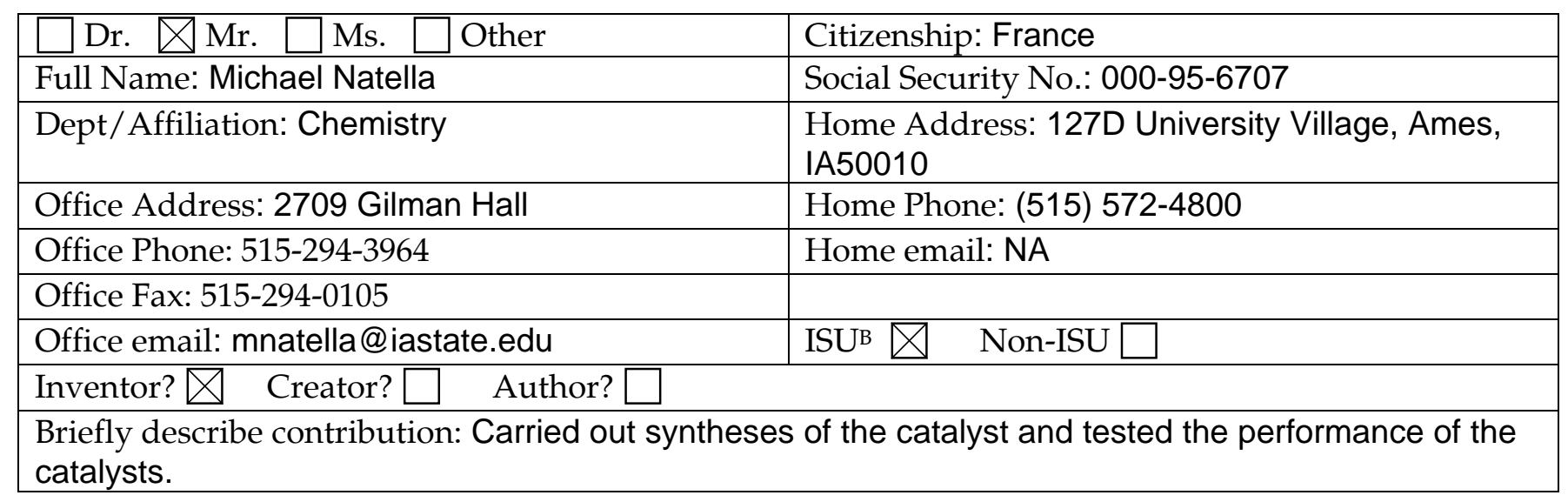

(Copy this section as needed for additional I/C/As.)

A CAVEAT - ISURF cannot guarantee that the order presented in the IPDR will result in an application or patent with names in the order originally presented. Further, the opportunity to provide an order of names does not imply in any or all of the purported inventors, a property right in having the names in a particular order.

В ISU I/C/A: An ISU I/C/A is a faculty member, staff member, student, visiting scientist, or visiting scholar that made his/her contribution to the invention/creation while at ISU, even if the person is no longer associated with ISU. A current ISU employee that made his/her contribution while employed elsewhere is NOT considered an ISU I/C/A.

Designate an individual to serve as the main contact for this disclosure: Professor John Verkade

C. Type of Work: (Check all that apply.)

$\bigotimes$ Invention $\square$ Biological Material $\square$ Software $\square$ Video $\square$ Multimedia $\square$ Other

\section{Status of Invention/Creation:}

Do you have data showing that the invention/creation actually works as intended?

$\bigotimes$ Yes $\quad \square$ No, but have results $\quad \square$ No, only an idea at this time

If no, when would be a good time to check on your progress? 
In terms of technical viability for commercialization, at what stage do you think your invention/creation is? (choose one)

$\square$ Very early (needs a lot of follow up work)

$\bigotimes$ Early (needs some work)

$\square$ Can be adopted right away

$\square$ Don't know

Have you built a working prototype or do you have a sample available?

$\triangle$ YES $\square$ NO

Applicable

$\square$ Not

If YES: Can you provide one? $\bigotimes$ YES $\square$ NO If yes, when could it be available? immediately

If NO: When do you estimate that a sample or working prototype will be available?

If you are not sure how to answer these questions, please call Lynne Mumm at 294-4742.

E.

Month/Year that the idea occurred

to you: $\underline{01 / 26 / 06 .}$

F. First Written Record

Date you first described the idea in writing or made a record of beginning the process to make the invention work.: $01 / 26 / 2006$.

Form of First Written Record. (What you wrote on, e.g. notebook (include number \& page), computer file, report, etc.): Yulin Huang's Lab Notebook \# 3, p. 02 \& p. 03

Was the record witnessed? $\square$ YES $\square$ NO

If yes, by whom? Brian G. Trewyn 
G. Public Disclosures: Have you told/written, or are you planning to tell/write, anybody about the invention/creation? (For example, abstracts, presentations, proceedings, publications, public use of the invention, or discussions with persons outside of ISU.) $\quad \square$ YES $\bigotimes$ NO

If yes, provide details of the EACH incidence, starting with the earliest:

\begin{tabular}{|l|l}
\hline Date: & Event (name of event, location, sponsor, etc.):
\end{tabular}

Please provide a copy of the material you presented or will be presenting. If you have a manuscript or abstract ready for submission describing the invention/creation, please forward a copy to us and identify the journal or conference where the manuscript will/did appear, including relevant dates. Be sure to include the dates and URL of any web publications, if you have them. Many times abstracts and articles are posted to the web prior to print distribution. If you have made, or are planning to make, any other public disclosures, be sure to provide information on those also.

Is there a thesis or dissertation (written or planned) that discloses any information about this invention? $\quad$ YES $\square$ NO

If yes, what is the student's name? Yulin Huang_ Projected graduation date? June, 2009

What is the status of the thesis/dissertation? In preparation

It is very important that you inform us if you make plans for any additional public disclosures of this invention/creation after submitting this form!!

H. Funding Information: Through contracts with the University, most sponsors have obtained intellectual property rights and thus require notification when an invention or a creation is made. It is IMPORTANT, therefore, that ALL sources of funding utilized in the conception, creation, or enablement of the invention/creation, including inventors' salaries, be reported to this office. You are therefore REQUIRED to disclose ALL external funding sources below:

Federally Funded Projects:

\begin{tabular}{|l|l|l|}
\hline Agency & Agency Contract No(s). & ISU Account No(s). \\
\hline$\square$ DOC & & \\
\hline$\square$ DOE & DE-FC26-06NT43023 & $\begin{array}{l}\text { 401-25-12 (Contact: Becky } \\
\text { Staedtler in CSET) }\end{array}$ \\
\hline
\end{tabular}




\begin{tabular}{|l|l|l|}
\hline$\square$ EPA & & \\
\hline$\square$ NASA & & \\
\hline$\square$ NIH & & \\
\hline$\square$ NSF & & \\
\hline$\square$ USDA & & \\
\hline$\square$ USB & & \\
Give Name: & & \\
\hline
\end{tabular}

IAHEES Projects: Project No(s)::

Source (if known): $\square$ Animal Health $\square$ Hatch Formula Funds

$\square$ McIntire-Stennis $\square$ Multi-State Research

IPRT Projects: $\square$ Ames Lab/DOE Contract No. W-7405-Eng-82 B\&R Code (required):

$\square$ IPRT Project No.
$\square$ CATD Project No.

OTHER ENTITIES: Companies, Commodity Groups or Research Consortia:

\begin{tabular}{|l|l|l|l|l|l|}
\hline & & \multirow{2}{*}{ Entity Name } & ISU Account No(s). & Entity Contract No(s). & \multicolumn{3}{|l|}{ Is there an agreement? } \\
\cline { 4 - 7 } & & & Yes & No & Not Sure \\
\hline
\end{tabular}

*If yes, attach a copy of the agreement to this form if you have one.

$\square$ NO EXTERNAL FUNDING received for the project (Note that ISU accounts beginning with "700", “701", and "704" are internal, not external, funds).

I. Did you use materials or data (biological or chemical) from other labs (ISU or outside ISU) which are not public domain materials, or which you are unsure as to their proprietary nature?

$\square$ YES $\square$ NO

If yes, identify the materials and their source:

If the materials are subject to a Material Transfer Agreement (MTA) or other agreement, attach a copy.

J. Brief Description: (i.e., What is it? What does it do? What is it for? Why was it invented/created? etc.) 
Our invention provides a series of methods to synthesize mesoporous oxide-supported metal catalyst system for conversion of synthetic gas to alcohols. Such metal catalysts in mesoporous oxide supports framework offer several advantages: 1 . The active catalytic sites are metal nanoparticles with controllable particle sizes. 2 . We have demonstrated that the catalytic efficiency can be dramatically improved by controlling the particle size of metal nanoparticle inside the high-surface-area mesoporous oxide matrix, such as mesoporous silica and titania. We also showed that the catalytic sites are homogeneously distributed through the mesoporous framework. 3. These heterogeneous catalysts that can be easily separated from the reaction mixture by filtration or centrifuge. The isolated catalyst materials can be easily recycled for utilization in subsequent reactions. 4. The cost for the synthesis of these catalysts is significantly economical, and these materials are made with commercially available resources. 5 . The catalyst material is thermally and chemically stable. 6 . The nanoscale pores ( $>2 \mathrm{~nm}$ in diameter) allow efficient passage of reactants and products. 7. Our product converts syngas into alcohols under mild reaction conditions.

What are some keywords that describe your invention/creation that will allow your technology to be searched for commercial purposes? Mesoporous oxide, metal catalyst, nanoparticles, synthetic gas, alcohols, ethanol.

K. Detailed Description: Before we can make an assessment of the commercial value and possible protection of your invention/creation we must have your data proving that the new invention/creation actually works ("enabling data"), and also a detailed description of the materials and methods you used to collect the data. This information should be sufficient to "teach" the invention. Please include a copy of your "enabling data" and "materials and methods". This can be in the form of a manuscript attached under Section G. See separate electronic attachment.

L. Does your invention need/consist of one or more SOFTWARE PROGRAMS or COMPUTERIZED DATABASES? $\square$ YES $\square$ NO (If NO, please skip to section $\mathbf{M}$ )

If Yes, please complete steps 1-3.

1. a) If you are an Ames Lab employee: Projects of this nature funded under the Ames Laboratory Contract with DOE must receive permission from DOE to assert Copyright. Once we receive permission to assert copyright, we will contact you with the appropriate copyright statement and DOE acknowledgement..

1. b) If are not an Ames Lab employee: Put the following statement on the "first screen" in the program/database and on the label of any disk or CD which you use to transfer the program/database:

$$
\begin{gathered}
\text { [Name of Program/Database] } \\
\text { by } \\
\text { Name(s) of Creator(s) }
\end{gathered}
$$

Copyright (C) - [Year of First Publication] ${ }^{* *}$ Iowa State University Research Foundation, Inc. 
**If unpublished: "Unpublished Work Copyright (C [Year of Creation]"

2) Send us a copy (a disk and a printout (if practical) of the source code, object code and documentation) of each of the programs or databases. We may need to register your programs/databases for copyright protection.

3) Complete and return the Additional Information for Disclosure of Copyright and Multimedia Works form. This form is available at http://www.iastate.edu/ isurf/forms/ipdr.html or from Lynne Mumm.

M. Prior Art: To determine whether we can protect your invention/creation, it will be necessary to compare it to what is already known or available. Please provide the following information to the best of your ability.

1. What is the deficiency in the prior art that your invention/creation improves upon, and/or the limitation it extends? (i.e. It works faster; is cheaper to make; produces less toxic wastes, etc.)

It is well known that the current state-of-the-art methods for syngas conversion struggles with many problems, such as low product selectivity, catalyst leaching, and low reactivity and recyclability. Our co-condensation method to synthesize mesoporous oxide-supported metal catalyst systems allows the catalytic sites to be encapsulated in the framework of high-surfacearea mesoporous oxide, which makes the catalytic sites very stable in the support without leaching. The catalytic sites are distributed homogeneously in the mesoporous oxide materials. Our catalysts are economical in cost, and they can be recycled and reused.

2. If possible, please provide copies of or references to the prior art (including patents, journal articles, book chapters, news releases, meeting abstracts, names of persons, etc.)

N. What are some other COMPETING invention/creation(s) and how do they compare with yours?

1. What are the advantages of your invention/creation compared to the competing inventions/creations?

The catalytic performance of our catalyst system is superior to other known competing

heterogeneous systems. Particularly, our catalysts showed an unprecedented high

selectivity for the desired alcohol products. Also, the high recyclability and low cost of

preparation make our catalyst the most economical system available. 
2. In as much detail as possible, describe what the invention does, as opposed to how it does it. Please include quantifiable differences between the new invention/creation and what is commercially available. (i.e. why did you invent this? If it is less expensive, by how much? If it is more efficient, by what measure and how much? If it is more environmentally friendly, provide explanation.)

Our catalyst system allows a rapid reaction to occur under mild reaction conditions. The selectivity of our catalysts for producing the desired alcohol products (ethanol and propanol known as liquid fuels) is significantly higher than those of other known catalytic systems. Also, the cost of our catalysts is significantly cheaper than those of other catalysts.

O. Why do you believe your solution would NOT have been obvious to another researcher working on the same problem?

Due to our unique expertise for inorganic nanomaterials, we were able to design a catalyst that takes advantage of the best properties of both metal nanoparticles and mesoporous oxide materials.

\section{P. Potential Market}

1. What do you see as the most likely COMMERCIAL use(s) of your invention/creation? (Update us when you think of new uses.)

\section{Alternative energy/fuel sources}

2. How do you think your invention can be commercialized?

$\bigotimes$ As stand-alone technology $\square$ Both

$\square$ Combined with other technology $\quad \square$ Not Sure

3. To the best of your knowledge, how big is the potential market of your invention?

$\square$ Very large (> \$1 billion) $\square$ Small (<\$50 million)

$\bigotimes$ Large (in between) $\square$ Not Sure

4. What types of commercial or governmental entities do you think may be interested in your invention/creation? If you can identify such entities specifically, please do so. (Update us when you think of new firms or types of companies.)

Energy companies, petrochemical companies, chemical companies.

5. Are there reasons that you think commercial or governmental entities might not be interested in your invention?

At this point there are still some developments that must be made, e.g., scale-up synthesis 
Q. Signatures and Assignment of all ISU Inventor(s)/Creator(s)/Author(s): All ISU $\mathrm{I} / \mathrm{C} / \mathrm{As}$ must sign and have their signature witnessed at the time of signature. The witness must not be directly involved in the invention/creation. (Copy this section as needed.)

\section{ISU I/C/As}

Signature:

Printed Name:

Victor Shang-Yi Lin

Trewyn

Date: $7 / 31 / 07$

Signature:

Printed Name: Yulin Huang

Date:

$7 / 31 / 07$

Signature:

Printed Name:

Yang Cai

Date:

$7 / 31 / 07$

Signature:

Printed Name: Michael Natella

Date: $7 / 31 / 07$

\section{Witness}

Signature:

Printed Name: Brian G.

Date: $7 / 31 / 07$

Signature:

Printed Name:

Date:

Signature:

Printed Name:

Date:

Signature:

Printed Name:

Date:

By the signature(s) above and in accordance to Iowa State University policies, the individual(s) hereby assigns to ISURF all intellectual property rights, titles, and interests in this invention/creation.

R. Signatures of Non- ISU Inventor(s)/Creator(s)/Author(s). (Copy this section as needed.)

Non-ISU I/C/As

Signature: $\underline{\text { Witness }}$

Signature: 
Printed Name:

Date:

Signature:

Printed Name:

Date:

S. For Ames Laboratory Office of Intellectual Property Use Only:
Printed Name:

Date:

Signature:

Printed Name:

Date:

Date: 